\title{
THE HERSCHEL ORION PROTOSTAR SURVEY: SPECTRAL ENERGY DISTRIBUTIONS AND FITS USING A GRID OF PROTOSTELLAR MODELS
}

\author{
E. Furlan ${ }^{1}$, W. J. Fischer ${ }^{2,14}$, B. Ali ${ }^{3}$, A. M. Stutz ${ }^{4}$, T. Stanke ${ }^{5}$, J. J. Tobin ${ }^{6,15,16}$, S. T. Megeath ${ }^{7}$, M. Osorio ${ }^{8}$, \\ L. Hartmann ${ }^{9}$, N. Calvet $^{9}$, C. A. Poteet ${ }^{10}$, J. Booker ${ }^{7}$, P. Manoj ${ }^{11}$, D. M. Watson ${ }^{12}$, And L. Allen ${ }^{13}$ \\ ${ }^{1}$ Infrared Processing and Analysis Center, California Institute of Technology, 770 S. Wilson Ave., Pasadena, CA 91125, USA; furlan@ipac.caltech.edu \\ ${ }^{2}$ Goddard Space Flight Center, 8800 Greenbelt Road, Greenbelt, MD 20771, USA \\ ${ }^{3}$ Space Science Institute, 4750 Walnut Street, Boulder, CO 80301, USA \\ ${ }^{4}$ Max-Planck-Institut für Astronomie, Königstuhl 17, D-69117 Heidelberg, Germany \\ ${ }^{5}$ ESO, Karl-Schwarzschild-Strasse 2, D-85748 Garching bei München, Germany \\ ${ }^{6}$ National Radio Astronomy Observatory, Charlottesville, VA 22903, USA \\ ${ }^{7}$ Ritter Astrophysical Research Center, Department of Physics and Astronomy, University of Toledo, 2801 W. Bancroft Street, Toledo, OH 43606, USA \\ ${ }^{8}$ Instituto de Astrofísica de Andalucía, CSIC, Camino Bajo de Huétor 50, E-18008 Granada, Spain \\ ${ }^{9}$ Department of Astronomy, University of Michigan, 500 Church Street, Ann Arbor, MI 48109, USA \\ ${ }^{10}$ New York Center for Astrobiology, Rensselaer Polytechnic Institute, 110 Eighth Street, Troy, NY 12180, USA \\ ${ }^{11}$ Department of Astronomy and Astrophysics, Tata Institute of Fundamental Research, Homi Bhabha Road, Colaba, Mumbai 400005, India \\ ${ }_{12}$ Department of Physics and Astronomy, University of Rochester, Rochester, NY 14627, USA \\ ${ }^{13}$ National Optical Astronomy Observatory, 950 N. Cherry Avenue, Tucson, AZ 85719, USA \\ Received 2015 August 21; accepted 2016 February 21; published 2016 May 6
}

\begin{abstract}
We present key results from the Herschel Orion Protostar Survey: spectral energy distributions (SEDs) and model fits of 330 young stellar objects, predominantly protostars, in the Orion molecular clouds. This is the largest sample of protostars studied in a single, nearby star formation complex. With near-infrared photometry from 2MASS, midand far-infrared data from Spitzer and Herschel, and submillimeter photometry from APEX, our SEDs cover $1.2-870 \mu \mathrm{m}$ and sample the peak of the protostellar envelope emission at $\sim 100 \mu \mathrm{m}$. Using mid-IR spectral indices and bolometric temperatures, we classify our sample into 92 Class 0 protostars, 125 Class I protostars, 102 flatspectrum sources, and 11 Class II pre-main-sequence stars. We implement a simple protostellar model (including a disk in an infalling envelope with outflow cavities) to generate a grid of 30,400 model SEDs and use it to determine the best-fit model parameters for each protostar. We argue that far-IR data are essential for accurate constraints on protostellar envelope properties. We find that most protostars, and in particular the flat-spectrum sources, are well fit. The median envelope density and median inclination angle decrease from Class 0 to Class I to flatspectrum protostars, despite the broad range in best-fit parameters in each of the three categories. We also discuss degeneracies in our model parameters. Our results confirm that the different protostellar classes generally correspond to an evolutionary sequence with a decreasing envelope infall rate, but the inclination angle also plays a role in the appearance, and thus interpretation, of the SEDs.
\end{abstract}

Key words: circumstellar matter - infrared: stars - methods: data analysis - stars: formation - stars: protostars

Supporting material: figure set, machine-readable tables

\section{INTRODUCTION}

The formation process of low- to intermediate-mass stars is divided into several stages, ranging from the deeply embedded protostellar stage to the period when a young star is dispersing its protoplanetary disk in which planets may have formed. During the protostellar phase, which is estimated to last $\sim 0.5 \mathrm{Myr}$ (Evans et al. 2009; Dunham et al. 2014), the growing central source accretes dust and gas from a collapsing envelope. The material from the envelope is most likely accreted through a disk, feeding the growing star. A fraction of the mass is ejected in outflows, which carve openings into the envelope along the outflow axis. Despite our understanding of the basic processes operating in low-mass protostars, fundamental questions remain (e.g., Dunham et al. 2014). In particular, it is not understood how the processes of infall, feedback from outflows, disk accretion, and the surrounding birth environment affect mass accretion and determine the

\footnotetext{
14 NASA Postdoctoral Program Fellow.

15 Hubble Fellow.

16 Current address: Leiden Observatory, Leiden University, P.O. Box 9513, 2300-RA Leiden, The Netherlands.
}

ultimate stellar mass. The luminosity of protostars, which can be dominated by accretion, is observed to span more than three orders of magnitude, yet the underlying physics of this luminosity range is also not understood (Dunham et al. 2010; Offner \& McKee 2011). It is in this protostellar phase that disks are formed, setting the stage for planet formation, yet how infall, feedback, accretion, and environment influence the properties of disks and of planets that eventually form from them is unknown. The large samples of well-characterized protostars identified from surveys with Spitzer and Herschel now provide the means to systematically study the processes controlling the formation of stars and disks; the goal of this work is to provide such a characterization for the protostars found in the Orion A and B clouds, the largest population of protostars for any of the molecular clouds within $500 \mathrm{pc}$ of the Sun (Kryukova et al. 2012; Dunham et al. 2013, 2015).

In protostars, dust in the disk and envelope reprocesses the shorter-wavelength radiation emitted by the central protostar and the accretion shock on the stellar surface and reemits it prominently at mid- to far-infrared wavelengths. As a result, the combined emission of most protostellar systems (consisting of protostar, disk, and envelope) peaks in the far-IR. Young, 
deeply embedded protostars have spectral energy distributions (SEDs) with steeply rising slopes in the infrared, peaking around $100 \mu \mathrm{m}$, and large fractional submillimeter luminosities (e.g., Enoch et al. 2009; Stutz et al. 2013). Near 10 and $18 \mu \mathrm{m}$, absorption by sub-micron-sized silicate grains causes broad absorption features; in addition, there are several ice absorption features across the infrared spectral range (Boogert et al. 2008; Pontoppidan et al. 2008). These absorption features are indicative of the amount of material along the line of sight, with the deepest features found for the most embedded objects. In addition, owing to the asymmetric radiation field, the orientation of a protostellar system to the line of sight, whether through a dense disk or a low-density cavity, plays a role in the appearance of the SED. It influences the near- to far-IR slope, the depth of the silicate feature, the emission peak, and the fraction of light emitted at the longest wavelengths (see, e.g., Whitney et al. 2003b).

To classify young stellar objects (YSOs) into observational classes, the near- to mid-infrared spectral index $n\left(\lambda F_{\lambda} \propto \lambda^{n}\right)$ from about 2 to $20 \mu \mathrm{m}$ has traditionally been used (Adams et al. 1987; Lada 1987; André \& Montmerle 1994; Evans et al. 2009; Dunham et al. 2014). This index is positive for a Class 0/I protostar, between -0.3 and 0.3 for a flat-spectrum source, and between -1.6 and -0.3 for a Class II pre-main-sequence star. Class 0 protostars are distinguished from Class I protostars as having $L_{\text {submm }} / L_{\text {bol }}$ ratios larger than $0.5 \%$, according to the original definition by André et al. (1993). Other values for this threshold that have recently been used are 1\% (Sadavoy et al. 2014) and even 3\% (Maury et al. 2011). Another measure for the evolution of a young star is the bolometric temperature $\left(T_{\mathrm{bol}}\right)$, which is the temperature of a blackbody with the same flux-weighted mean frequency as the observed SED (Myers \& Ladd 1993). A Class 0 protostar has $T_{\text {bol }}<70 \mathrm{~K}$, a Class I protostar $70 \mathrm{~K}<T_{\text {bol }}<650 \mathrm{~K}$, and a Class II pre-mainsequence star $650 \mathrm{~K}<T_{\text {bol }}<2800 \mathrm{~K}$ (Chen et al. 1995). These observational classes are inferred to reflect evolutionary stages, with the inclination angle to the line of sight being the major source of uncertainty in translating classes to "stages" (Robitaille et al. 2006; Evans et al. 2009). Also the accretion history, which likely includes episodic accretion events and thus temporary increases in luminosity, adds to this uncertainty (Dunham et al. 2010; Dunham \& Vorobyov 2012). Protostars with infalling envelopes of gas and dust correspond to Stages 0 and I, with the transition from Stage 0 to I occurring when the stellar mass becomes larger than the envelope mass (Dunham et al. 2014). Young stars that have dispersed their envelopes and are surrounded by circumstellar disks correspond to Stage II.

By modeling the SEDs of protostars, properties of their envelopes, and to some extent of their disks, can be constrained. The near-IR is particularly sensitive to extinction and thus constrains the inclination angle and cavity opening angle, as well as the envelope density. Mid-IR spectroscopy reveals the detailed emission around the silicate absorption feature and thus provides additional constraints for both disk and envelope properties (see, e.g., Furlan et al. 2008). At longer wavelengths, envelope emission starts to dominate. Thus, photometry in the far-IR is necessary to determine the peak of the SED and constrain the total luminosity and envelope properties.

Here we present $1.2-870 \mu \mathrm{m}$ SEDs and radiative transfer model fits of 330 YSOs, most of them protostars, in the Orion star formation complex. This is the largest sample of protostars studied in a single, nearby star-forming region (distance of 420 pc; Menten et al. 2007; Kim et al. 2008) and therefore significant for advancing our understanding of protostellar structure and evolution. These protostars were identified in Spitzer Space Telescope (Werner et al. 2004) data by Megeath et al. (2012) and were observed at 70 and $160 \mu \mathrm{m}$ with the Photodetector Array Camera and Spectrometer (PACS; Poglitsch et al. 2010) on the Herschel Space Observatory ${ }^{17}$ (Pilbratt et al. 2010) as part of the Herschel Orion Protostar Survey (HOPS), a Herschel open-time key program (e.g., Fischer et al. 2010; Stanke et al. 2010; Manoj et al. 2013; Stutz et al. 2013; B. Ali et al. 2016, in preparation; W. J. Fischer et al. 2016, in preparation). To extend the SEDs into the submillimeter, most of the YSOs were also observed in the continuum at 350 and $870 \mu \mathrm{m}$ with the Atacama Pathfinder Experiment (APEX) telescope (Stutz et al. 2013). Our sample also includes 16 new protostars identified in PACS data obtained by the HOPS program (Stutz et al. 2013; Tobin et al. 2015; see Section 2). We use a grid of 30,400 protostellar model SEDs to find the best fit to the SED for each object and constrain its protostellar properties. As mentioned above, the far-infrared data add crucial constraints for the model fits, given that for most protostars the SED peaks in this wavelength region, and therefore, within the framework of the model grid, our SED fits yield the most reliable protostellar parameters to date for these sources.

\section{SAMPLE DESCRIPTION}

The 488 protostars identified in Spitzer data by Megeath et al. (2012) represent the basis for the HOPS sample ${ }^{18}$ (see Fischer et al. 2013; Manoj et al. 2013; Stutz et al. 2013). They have $3.6-24 \mu \mathrm{m}$ spectral indices $\geqslant-0.3$ and thus encompass flat-spectrum sources. To be included in the target list for the PACS observations, the predicted flux of a protostar in the $70 \mu \mathrm{m}$ PACS band had to be at least $42 \mathrm{mJy}$ as extrapolated from the Spitzer SED. Since targets were required to have a $24 \mu \mathrm{m}$ detection, protostars in the Orion Nebula-where the Spitzer $24 \mu \mathrm{m}$ are saturated-are excluded. In addition, after the PACS data were obtained, several new point sources that were very faint or undetected in the Spitzer bands were discovered in the Herschel data (Stutz et al. 2013). Fifteen of them were found to be reliable new protostars. One more protostar, which was not included in the sample of Stutz et al. (2013) owing to its more spatially extended appearance at $70 \mu \mathrm{m}$, was recently confirmed by Tobin et al. (2015). We have added these 16 protostars to the HOPS sample for this work (see Appendix C). Most of these new protostars have very red colors and are thus potentially the youngest protostars identified in Orion (see Stutz et al. 2013).

Each object in the target list was assigned a "HOPS" identification number, resulting in 410 objects with such numbers; HOPS 394 to 408 are the new protostars identified by Stutz et al. (2013), and HOPS 409 is the new protostar from Tobin et al. (2015). Four of the 410 HOPS targets turned out to

\footnotetext{
17 Herschel is an ESA space observatory with science instruments provided by European-led Principal Investigator consortia and with important participation from NASA.

18 The selection of HOPS targets is based on an earlier version of the Spitzer Orion Survey, and in addition some objects likely in transition between Stages I and II were included; thus, not all protostars in the HOPS sample are classified as protostars with envelopes in Megeath et al. (2012).
} 
be duplicates, and 31 are likely extragalactic contaminants (see Appendix C.2.2 for details). Some objects in the HOPS target list were not observed by PACS; of these 33 objects, 16 are likely contaminants, while the remaining objects were originally proposed but were not observed since they were too faint to have been detected with PACS in the awarded observing time. In addition, 35 HOPS targets were not detected at $70 \mu \mathrm{m}$ (see Appendices C.2.1 and C.2.2); eight of these are considered extragalactic contaminants, while two of them (HOPS 349 and 381) have only two measured flux values each, making their nature more uncertain. One more target, HOPS 350 , also has just two measured flux values (at 24 and $70 \mu \mathrm{m}$ ) and is therefore also excluded from the analysis of this paper. Similarly, we excluded HOPS 352, since it was only tentatively detected at $24 \mu \mathrm{m}$ (it lies on the Airy ring of HOPS 84) and in none of the other data sets.

To summarize, starting from the sample of 410 HOPS targets, but excluding likely contaminants and objects not observed or detected by PACS, there are 330 remaining objects that have Spitzer and Herschel data and are considered protostars (based on their Spitzer classification from Megeath et al. 2012). They form the sample studied in this work. Their SEDs are presented in the next section, and in later sections we show and discuss the results of SED fits for these targets. Their coordinates, SED properties, and classification, as well as their best-fit model parameter values, are listed in Table 1. The 41 likely protostars that lack PACS data (either not observed or not detected) are presented in Appendix C.2.1.

\section{SPECTRAL ENERGY DISTRIBUTIONS}

\subsection{Data}

In order to construct SEDs for our sample of 330 YSOs, we combined our own observations with data from the literature and existing catalogs. For the near-infrared photometry, we used $J, H$, and $K_{s}$ data from the Two Micron All Sky Survey (2MASS; Skrutskie et al. 2006). For the mid-infrared spectral region, we used Spitzer data from Kryukova et al. (2012) and Megeath et al. (2012): the Infrared Array Camera (IRAC; Fazio et al. 2004) provided 3.6, 4.5, 5.8, and $8.0 \mu \mathrm{m}$ photometry, while the Multiband Imaging Photometer for Spitzer (MIPS; Rieke et al. 2004) provided $24 \mu \mathrm{m}$ photometry. In addition, most of the YSOs in the HOPS sample were also observed with the Infrared Spectrograph (IRS; Houck et al. 2004) on Spitzer using the Short-Low (SL; 5.2-14 $\mu \mathrm{m}$ ) and Long-Low (LL; 14-38 $\mu \mathrm{m}$ ) modules, both with a spectral resolution of about 90 (see, e.g., Kim et al. [2016] for a description of IRS data reduction). Herschel PACS data at 70, 100, and $160 \mu \mathrm{m}$ yielded far-infrared photometric data points (B. Ali et al. 2016, in preparation; the $100 \mu \mathrm{m}$ data are from the Gould Belt Survey; e.g., André et al. 2010). Most YSOs were also observed at 350 and $870 \mu \mathrm{m}$ (see Stutz et al. 2013) by the APEX telescope using the SABOCA and LABOCA instruments (Siringo et al. 2009, 2010, respectively). Thus, our SEDs have well-sampled wavelength coverage from 1.2 to $870 \mu \mathrm{m}$; we did not include additional data from the literature in order to preserve a homogeneous data set for all the objects in our sample.

The aperture radius used for the photometry varies depending on the instrument and wave band. The photometry in the 2MASS catalog was derived from point-spread function (PSF) fits using data from $4^{\prime \prime}$ apertures around each object (see the Explanatory Supplement to the 2MASS All Sky Data Release and Extended Mission Products). Megeath et al. (2012) used an aperture radius of 2". 4 for IRAC and PSF photometry for MIPS $24 \mu \mathrm{m}$ data. We used aperture radii of 9 !. 6 and sky annuli of 9!" $6-19$ ". 2 for PACS 70 and $100 \mu \mathrm{m}$ images; we then applied aperture correction factors of 0.7331 and 0.6944 to the 70 and $100 \mu \mathrm{m}$ fluxes, respectively. For PACS $160 \mu \mathrm{m}$, we used an aperture radius of 12 ". 8 , a sky annulus of 12 !" $8-25$ ". 6 , and an aperture correction factor of 0.6602 . In some cases (background contamination, close companions) we used PSF photometry at 70 and $160 \mu \mathrm{m}$ instead (see B. Ali et al. 2016, in preparation, for details). Finally, we adopted beam fluxes at 350 and $870 \mu \mathrm{m}$ (with FWHMs of 7!. 34 and 19", respectively). The IRS SL module has a slit width of 3".6, while the LL module is wider, with a slit width of 10 " 5 . Sometimes the flux level of the two segments did not match at $14 \mu \mathrm{m}$ (owing to slight mispointings or more extended emission from surrounding material measured in LL), and in these cases usually the SL spectrum was scaled by at most a factor of $\sim 1.4$ (typically 1.1-1.2). In a few cases, especially when the LL spectrum included substantial amounts of extended emission or flux from a nearby object, the LL spectrum was scaled down to match the flux level of the SL spectrum at $14 \mu \mathrm{m}$, typically by a factor of 0.8-0.9. We discuss how the different aperture sizes are accounted for in the model fluxes in Section 4.2.

The SEDs of our HOPS sample are shown in Figure 1 together with their best-fit models from our model grid (see sections below); the data are listed in Table 2. Many objects display a deep silicate absorption feature at $10 \mu \mathrm{m}$ and ice features in the 5-8 $\mu \mathrm{m}$ region, as expected for protostars. Those objects with very deep $10 \mu \mathrm{m}$ features and steeply rising SEDs are likely deeply embedded protostars, often seen at high inclination angles.

\subsection{Multiplicity and Variability}

A large fraction (203 out of 330) of the young stars in our sample have at least one Spitzer-detected source within a radius of 15 "; in most cases, this "companion" is faint in the infrared and likely a background star or galaxy. Thus, the emission at far-IR and submillimeter wavelengths is expected to be dominated by the protostar or pre-main-sequence star, and we can assume that the SEDs are representative of the YSOs even if the nearby sources cannot be separated at these wavelengths. There are a few YSOs that have objects separated by just $1^{\prime \prime}-3^{\prime \prime}$ and are only resolved in one or two IRAC bands (HOPS 22, 78, $108,184,203,247,293,364)$; in these cases we used the flux at the IRAC position that most closely matched those at longer wavelengths. We note that some of these very close "companions" are likely outflow knots. There are also unresolved binaries, which appear as single sources even in the IRAC observations (Kounkel et al. 2016); in these cases our SEDs show the combined flux in all wave bands. If two point sources are not fully resolved and the resulting blended source is elongated, no IRAC photometry was extracted. In such cases, a protostar may not have IRAC fluxes even though it was detected in the Spitzer images.

There are also several protostars that lie close to other protostars: HOPS 66 and $370(d=14$ ".9), HOPS 76 and 78 $(d=14$ !" 1$)$, HOPS 86 and $87(d=12$ !" 1$)$, HOPS 117 and 118 $(d=13 ! \cdot 7)$, HOPS 121 and $123(d=7 ! \cdot 6)$, HOPS 124 and 125 $(d=9$ !" 8$)$, HOPS 165 and $203(d=13$ !" 3$)$, HOPS 175 and 176 $(d=8$ "' 0$)$, HOPS 181 and $182(d=10$ "' 2$)$, HOPS 225 and 226 
Table 1

Classification and Best-fit Model Parameters for the HOPS Sample

\begin{tabular}{|c|c|c|c|c|c|c|c|c|c|c|c|c|c|c|c|}
\hline Object & $\begin{array}{l}\text { R.A. } \\
\left({ }^{\circ}\right) \\
(2)\end{array}$ & $\begin{array}{l}\text { Decl. } \\
\left({ }^{\circ}\right) \\
(3)\end{array}$ & $\begin{array}{l}\text { Class } \\
\text { (4) }\end{array}$ & $\begin{array}{l}L_{\mathrm{bol}} \\
\left(L_{\odot}\right) \\
(5)\end{array}$ & $\begin{array}{l}T_{\text {bol }} \\
(\mathrm{K}) \\
(6)\end{array}$ & $\begin{array}{c}n_{4.5-24} \\
(7)\end{array}$ & $\begin{array}{c}L_{\text {tot }} \\
\left(L_{\odot}\right) \\
(8)\end{array}$ & $\begin{array}{l}R_{\text {disk }} \\
\text { (au) } \\
\text { (9) }\end{array}$ & $\begin{array}{c}\rho_{1000} \\
\left(\mathrm{~g} \mathrm{~cm}^{-3}\right) \\
(10)\end{array}$ & $\begin{array}{l}M_{\text {env }} \\
\left(M_{\odot}\right) \\
(11)\end{array}$ & $\begin{array}{c}\theta \\
\left({ }^{\circ}\right) \\
(12)\end{array}$ & $\begin{array}{c}i \\
\left({ }^{\circ}\right) \\
(13)\end{array}$ & $\begin{array}{c}A_{V} \\
(\mathrm{mag}) \\
(14)\end{array}$ & $\begin{array}{l}\text { Scaling } \\
\text { Factor } \\
(15)\end{array}$ & (16) \\
\hline$\overline{\text { HOPS } 1}$ & 88.5514 & 1.7099 & I & 1.517 & 72.6 & 1.469 & 3.0 & 100 & $2.38 \times 10^{-19}$ & 0.0133 & 5 & 63 & 23.2 & 0.99 & 2.319 \\
\hline HOPS 2 & 88.5380 & 1.7144 & I & 0.542 & 356.5 & 0.455 & 1.3 & 5 & $2.38 \times 10^{-20}$ & 0.0012 & 15 & 32 & 13.1 & 1.30 & 2.476 \\
\hline HOPS 3 & 88.7374 & 1.7156 & flat & 0.553 & 467.5 & 0.260 & 0.820 & 5 & $1.19 \times 10^{-20}$ & 0.0007 & 5 & 50 & 3.0 & 0.81 & 3.331 \\
\hline HOPS 4 & 88.7240 & 1.7861 & I & 0.422 & 203.3 & 1.243 & 0.600 & 5 & $1.78 \times 10^{-19}$ & 0.0099 & 5 & 63 & 2.5 & 2.00 & 4.139 \\
\hline HOPS 5 & 88.6340 & 1.8020 & I & 0.390 & 187.1 & 0.626 & 1.6 & 50 & $2.38 \times 10^{-19}$ & 0.0096 & 35 & 63 & 12.4 & 0.52 & 2.459 \\
\hline HOPS 6 & 88.5767 & 1.8176 & I & 0.055 & 112.5 & 1.308 & 0.210 & 5 & $1.78 \times 10^{-20}$ & 0.0010 & 5 & 76 & 8.0 & 2.00 & 4.091 \\
\hline HOPS 7 & 88.5835 & 1.8452 & 0 & 0.528 & 58.0 & 1.707 & 6.1 & 100 & $1.78 \times 10^{-20}$ & 0.0010 & 15 & 81 & 18.7 & 2.00 & 2.981 \\
\hline HOPS 10 & 83.7875 & -5.9743 & 0 & 3.330 & 46.2 & 0.787 & 5.4 & 500 & $2.38 \times 10^{-18}$ & 0.135 & 5 & 70 & 0.0 & 1.77 & 3.168 \\
\hline HOPS 11 & 83.8059 & -5.9661 & 0 & 8.997 & 48.8 & 2.200 & 33.2 & 100 & $2.38 \times 10^{-18}$ & 0.115 & 25 & 63 & 39.8 & 1.10 & 3.385 \\
\hline HOPS 12 & 83.7858 & -5.9317 & 0 & 7.309 & 42.0 & 1.815 & 5.8 & 100 & $5.94 \times 10^{-18}$ & 0.332 & 5 & 32 & 0.0 & 1.91 & 2.207 \\
\hline HOPS 13 & 83.8523 & -5.9260 & flat & 1.146 & 383.6 & 0.208 & 2.4 & 5 & $5.94 \times 10^{-20}$ & 0.0031 & 15 & 18 & 15.2 & 0.78 & 2.149 \\
\hline HOPS 15 & 84.0792 & -5.9237 & flat & 0.171 & 342.0 & 0.116 & 0.600 & 50 & $2.38 \times 10^{-18}$ & 0.0745 & 45 & 63 & 9.0 & 2.00 & 3.329 \\
\hline HOPS 16 & 83.7534 & -5.9238 & flat & 0.682 & 361.0 & 0.019 & 3.0 & 5 & $1.78 \times 10^{-18}$ & 0.0548 & 45 & 18 & 25.4 & 0.99 & 2.464 \\
\hline HOPS 17 & 83.7799 & -5.8683 & I & 0.299 & 341.3 & 0.389 & 1.5 & 500 & $1.78 \times 10^{-19}$ & 0.0080 & 35 & 63 & 0.0 & 0.50 & 5.279 \\
\hline HOPS 18 & 83.7729 & -5.8651 & I & 1.419 & 71.8 & 0.743 & 5.2 & 50 & $1.78 \times 10^{-18}$ & 0.0851 & 25 & 76 & 1.1 & 0.51 & 4.915 \\
\hline HOPS 19 & 83.8583 & -5.8563 & flat & 0.188 & 101.6 & -0.098 & 0.150 & 500 & $1.19 \times 10^{-16}$ & 6.53 & 15 & 18 & 3.7 & 0.50 & 5.445 \\
\hline HOPS 20 & 83.3780 & -5.8447 & I & 1.231 & 94.8 & 2.226 & 1.6 & 5 & $5.94 \times 10^{-19}$ & 0.0329 & 5 & 76 & 7.3 & 0.54 & 5.333 \\
\hline HOPS 22 & 83.7522 & -5.8172 & II & 0.100 & 238.2 & 0.494 & 0.290 & 5 & $1.19 \times 10^{-20}$ & 0.0007 & 5 & 63 & 7.5 & 0.97 & 3.049 \\
\hline HOPS 24 & 83.6956 & -5.7475 & I & 0.095 & 288.9 & 0.438 & 0.150 & 50 & $1.78 \times 10^{-19}$ & 0.0099 & 5 & 57 & 3.2 & 0.50 & 3.998 \\
\hline HOPS 26 & 83.8223 & -5.7040 & II & 0.484 & 1124.9 & -0.400 & 1.1 & 5 & $1.78 \times 10^{-20}$ & 0.0007 & 35 & 70 & 0.0 & 1.10 & 3.291 \\
\hline HOPS 28 & 83.6971 & -5.6989 & 0 & 0.494 & 46.3 & 1.342 & 2.6 & 100 & $1.78 \times 10^{-18}$ & 0.0731 & 35 & 76 & 2.4 & 0.84 & 3.327 \\
\hline HOPS 29 & 83.7044 & -5.6950 & I & 1.916 & 148.2 & 0.687 & 6.1 & 500 & $1.19 \times 10^{-19}$ & 0.0044 & 45 & 63 & 3.8 & 0.60 & 4.113 \\
\hline HOPS 30 & 83.6836 & -5.6905 & I & 3.791 & 81.2 & 1.836 & 21.2 & 100 & $1.19 \times 10^{-17}$ & 0.381 & 45 & 57 & 39.5 & 0.70 & 2.494 \\
\hline HOPS 32 & 83.6477 & -5.6664 & 0 & 2.011 & 58.9 & 0.937 & 3.0 & 5 & $1.78 \times 10^{-18}$ & 0.0937 & 15 & 70 & 7.7 & 0.97 & 3.527 \\
\hline HOPS 33 & 83.6884 & -5.6658 & flat & 0.120 & 777.6 & -0.397 & 0.400 & 5 & $1.78 \times 10^{-19}$ & 0.0071 & 35 & 70 & 5.3 & 1.34 & 3.797 \\
\hline HOPS 36 & 83.6101 & -5.6279 & flat & 1.024 & 374.6 & 0.005 & 2.2 & 5 & $5.94 \times 10^{-20}$ & 0.0031 & 15 & 18 & 16.4 & 0.71 & 3.552 \\
\hline HOPS 38 & 83.7697 & -5.6201 & 0 & 0.246 & 58.5 & 0.935 & 2.0 & 5 & $1.78 \times 10^{-16}$ & 5.48 & 45 & 18 & 80.0 & 1.96 & 7.198 \\
\hline HOPS 40 & 83.7855 & -5.5998 & 0 & 2.694 & 38.1 & 1.247 & 6.1 & 100 & $2.38 \times 10^{-17}$ & 0.974 & 35 & 41 & 82.6 & 2.00 & 5.459 \\
\hline
\end{tabular}

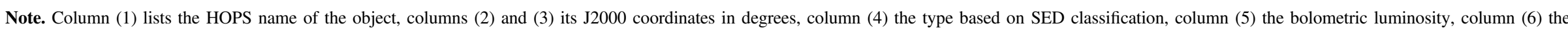

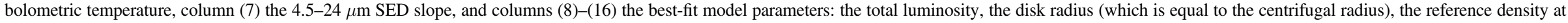

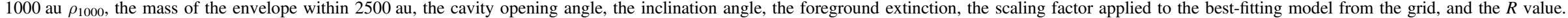

(This table is available in its entirety in machine-readable form.) 

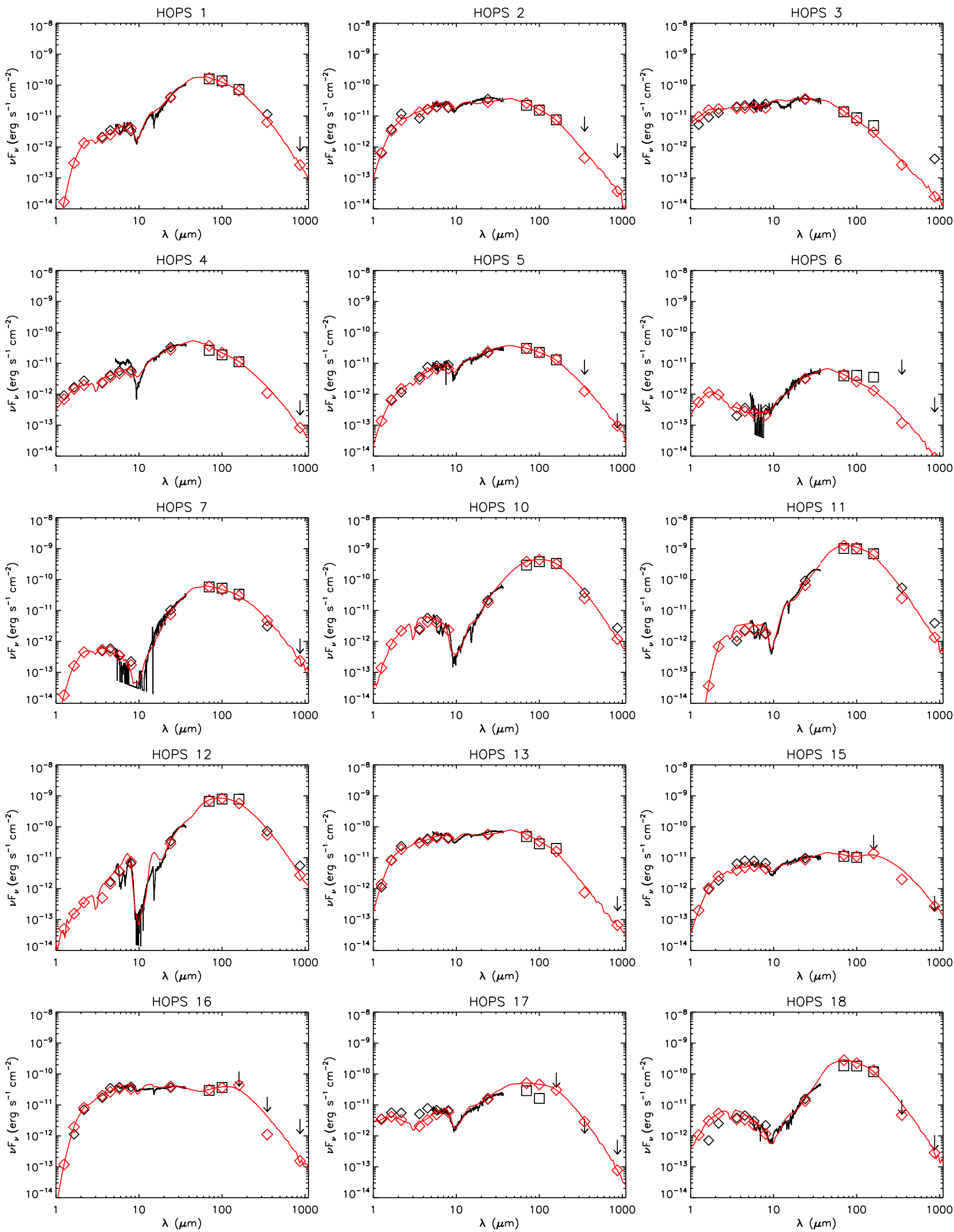

Figure 1. SEDs of the HOPS targets modeled in this work (black; open symbols: photometry, arrows: upper limits, line: IRS spectrum). The best-fit model for each object is shown as a red line, with fluxes taken from a $4^{\prime \prime}$ aperture for $\lambda<8 \mu \mathrm{m}$, a $5^{\prime \prime}$ aperture for $\lambda=8-37 \mu \mathrm{m}$, and a $10^{\prime \prime}$ aperture for $\lambda>37 \mu \mathrm{m}$. The red symbols are the model photometry measured in the same apertures and bandpasses as the data (see Section 4.2 for details). Only the first 15 SEDs are shown here.

(The complete figure set (22 images) is available online.) 
Table 2

SED Data for the HOPS Targets

\begin{tabular}{|c|c|c|c|c|c|c|c|c|c|c|c|}
\hline Object & $J$ Flux & $J$ Unc. & $J$ Flag & $\cdots$ & [70] Flux & [70] Unc. & [70] Flag & $\ldots$ & [870] Flux & [870] Unc. & [870] Flag \\
\hline HOPS 1 & $0.000 \mathrm{E}+00$ & $0.000 \mathrm{E}+00$ & 3 & $\ldots$ & $3.697 \mathrm{E}+00$ & $1.850 \mathrm{E}-01$ & 1 & $\ldots$ & $6.354 \mathrm{E}-01$ & $1.271 \mathrm{E}-01$ & 2 \\
\hline HOPS 2 & $2.770 \mathrm{E}-04$ & $5.000 \mathrm{E}-05$ & 1 & $\ldots$ & $5.188 \mathrm{E}-01$ & $2.617 \mathrm{E}-02$ & 1 & $\ldots$ & $3.865 \mathrm{E}-01$ & $7.730 \mathrm{E}-02$ & 2 \\
\hline HOPS 3 & $2.198 \mathrm{E}-03$ & $8.900 \mathrm{E}-05$ & 1 & $\ldots$ & $3.187 \mathrm{E}-01$ & $1.622 \mathrm{E}-02$ & 1 & $\ldots$ & $1.201 \mathrm{E}-01$ & $2.402 \mathrm{E}-02$ & 1 \\
\hline HOPS 4 & $3.820 \mathrm{E}-04$ & $5.300 \mathrm{E}-05$ & 1 & $\ldots$ & 6.116E-01 & $3.083 \mathrm{E}-02$ & 1 & $\ldots$ & $1.840 \mathrm{E}-01$ & $3.680 \mathrm{E}-02$ & 2 \\
\hline HOPS 5 & $0.000 \mathrm{E}+00$ & $0.000 \mathrm{E}+00$ & 3 & $\ldots$ & 7.103E-01 & $3.573 \mathrm{E}-02$ & 1 & $\ldots$ & $6.973 \mathrm{E}-02$ & $1.395 \mathrm{E}-02$ & 2 \\
\hline HOPS 6 & $0.000 \mathrm{E}+00$ & $0.000 \mathrm{E}+00$ & 3 & $\ldots$ & $9.110 \mathrm{E}-02$ & $5.523 \mathrm{E}-03$ & 1 & $\ldots$ & $2.311 \mathrm{E}-01$ & $4.622 \mathrm{E}-02$ & 2 \\
\hline HOPS 7 & $0.000 \mathrm{E}+00$ & $0.000 \mathrm{E}+00$ & 3 & $\cdots$ & $1.342 \mathrm{E}+00$ & $6.728 \mathrm{E}-02$ & 1 & $\cdots$ & $3.577 \mathrm{E}-01$ & $7.154 \mathrm{E}-02$ & 2 \\
\hline Object & & [5.4] Flux & & & & $\cdots$ & [35] Flux & & [35] Unc. & & IRS Scaling \\
\hline HOPS 1 & & $8.631 \mathrm{E}-03$ & & & & $\ldots$ & $1.185 \mathrm{E}+00$ & & $6.460 \mathrm{E}-02$ & & 1.17 \\
\hline HOPS 2 & & $4.360 \mathrm{E}-02$ & & & & $\ldots$ & $3.704 \mathrm{E}-01$ & & 3.035E-02 & & 1.00 \\
\hline HOPS 3 & & $4.460 \mathrm{E}-02$ & & & & $\ldots$ & $4.050 \mathrm{E}-01$ & & $2.443 \mathrm{E}-02$ & & 1.66 \\
\hline HOPS 4 & & $2.055 \mathrm{E}-02$ & & & & $\ldots$ & 4.943E-01 & & $3.484 \mathrm{E}-02$ & & 1.00 \\
\hline HOPS 5 & & $1.475 \mathrm{E}-02$ & & & & $\ldots$ & 3.077E-01 & & $4.484 \mathrm{E}-03$ & & 1.00 \\
\hline HOPS 6 & & $1.271 \mathrm{E}-03$ & & & & $\ldots$ & $5.350 \mathrm{E}-02$ & & $5.244 \mathrm{E}-03$ & & 1.00 \\
\hline HOPS 7 & & $6.459 \mathrm{E}-04$ & & & & $\ldots$ & $3.258 \mathrm{E}-01$ & & $2.114 \mathrm{E}-02$ & & 1.00 \\
\hline
\end{tabular}

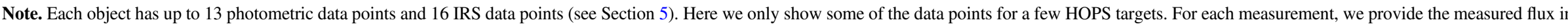

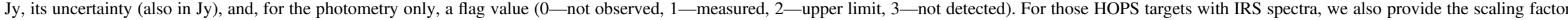

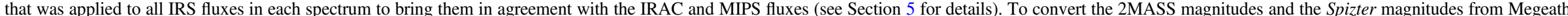

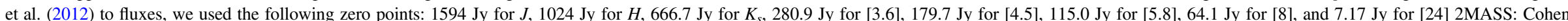
et al. 2003; IRAC: Reach et al. 2005; MIPS: Engelbracht et al. 2007.

(This table is available in its entirety in machine-readable form.) 
$(d=9 ! \cdot 2)$, HOPS 239 and $241(d=12 ! 4)$, HOPS 262 and 263 $(d=6 " .3)$, HOPS 316 and $358(d=6$ ". 9$)$, HOPS 332 and 390 $(d=11 ! 2)$, HOPS 340 and $341(d=4 ! \cdot 7)$, and HOPS 386 and $387(d=9$ !"9). HOPS 105 lies 8 "' 7 to the north of an infraredbright source, identified by Megeath et al. (2012) as a young star with a protoplanetary disk. This source is brighter than HOPS 105 in all Spitzer bands and at $70 \mu \mathrm{m}$, but it is well separated at all wavelengths. A similar situation applies to HOPS 128, which has a disk-dominated source 6." 3 to the southeast. HOPS 108 is 6". 6 from HOPS 64, which is brighter than HOPS 108 out to $8 \mu \mathrm{m}$, but not detected in the far-IR and submillimeter. HOPS 108 also lies 16". 6 from HOPS 369 and 28 ". 2 from HOPS 370. HOPS 140 has two neighboring sources, at 9!" 6 and $13 . " 9$, that are likely surrounded by protoplanetary disks; they are both brighter than HOPS 140 out to $8 \mu \mathrm{m}$, but at $70 \mu \mathrm{m}$ and beyond HOPS 140 dominates. HOPS 144 lies 7!"9 from HOPS 377; there is also a somewhat fainter, red source $11^{\prime \prime} 7$ to the northeast, which is not detected beyond $24 \mu \mathrm{m}$. This source also lies $9 ! 7$ to the southwest of HOPS 143. HOPS 173 forms a small cluster with HOPS 174 (at 7!"1) and HOPS 380 (at 11."4); HOPS 174 is the brightest source out to $24 \mu \mathrm{m}$, but at $70 \mu \mathrm{m}$ HOPS 173 takes on this role. Also HOPS 322, 323, and 389 form a group of protostars; HOPS 322 lies 13!" 4 from HOPS 389 and 20". 1 from HOPS 323, while HOPS 323 and 389 are 10". 2 apart. HOPS 323 is the brightest source.

Thus, there are 45 targets in our sample that have an object within $15^{\prime \prime}$ that is bright in the mid- or far-IR and that is resolved with IRAC and MIPS. Given that Megeath et al. (2012) used PSF photometry for the MIPS $24 \mu \mathrm{m}$ observations, they obtained reliable fluxes even for companions separated by less than 6", the typical PSF FWHM. For fluxes at 70 and $160 \mu \mathrm{m}$, we also used PSF photometry for objects that were point sources, but too close for aperture photometry. In cases where the fluxes could not be determined even with PSF photometry, we had to adopt upper limits instead. Similarly, we performed PSF photometry on protostars without companions, but contaminated by extended or filamentary emission; if the PSF photometry did not return a good fit, we used the flux value from aperture photometry as an upper limit.

Since most of our targets have an IRS spectrum, in addition to data points from IRAC at 5.8 and $8 \mu \mathrm{m}$ and from MIPS at $24 \mu \mathrm{m}$, we can detect discrepancies if flux values at similar wavelengths, but from different instruments, do not agree. They might be due to calibration or extraction problems in the IRS spectrum (for example, some extended emission around the target or a close companion), but also to variability. We assumed the former scenario if the flux deviations between IRS and IRAC and between IRS and MIPS were similar (and more than $10 \%$, a conservative estimate for the typical calibration uncertainty), and in such cases scaled the IRS spectrum to the MIPS $24 \mu \mathrm{m}$ flux. Even though this scaling could mask actual variability, it creates a representative SED for the YSO and yields an estimate of the protostellar parameters from model fits of the SED.

In Appendix A we identify potentially variable HOPS targets based on their mid-IR fluxes and find that about $5 \%$ of the protostars with IRS, IRAC, and MIPS data could be variable. The Young Stellar Object Variability (YSOVAR) program, which monitored large samples of protostars and pre-mainsequence stars in nearby star-forming regions with Spitzer at 3.6 and $4.5 \mu \mathrm{m}$ (Morales-Calderón et al. 2011; Cody et al. 2014; Günther et al. 2014; Rebull et al. 2014, 2015; Poppenhaeger et al. 2015; Wolk et al. 2015), found that up to $\sim 90 \%$ of flat-spectrum and Class I YSOs are variable on a timescale of days, with typical changes in brightness of $10 \%-$ $20 \%$. On longer timescales (years as opposed to days), 20\%$40 \%$ of members of young clusters show long-term variability, with the highest fraction for those clusters with more Class I protostars (Rebull et al. 2014). In Orion, the fraction of variable Class I protostars is $\sim 85 \%$ (Morales-Calderón et al. 2011). Using a larger sample of protostars in Orion and IRAC data at 3.6, 4.5, 5.8, and $8.0 \mu \mathrm{m}$, Megeath et al. (2012) found that, on a timescale of about 6 months, $60 \%-70 \%$ of Orion protostars show brightness variabilities of $\sim 20 \%$, with some as high as a factor of four. Thus, given that our SEDs consist of noncontemporaneous data sets, small flux discrepancies should be common, but we also expect some protostars with large mismatches.

One protostar with a large discrepancy between various data sets is HOPS 223. It is an outbursting protostar (also known as V2775 Ori; Caratti o Garatti et al. 2011), and for its SED we had 2MASS, IRAC, and MIPS data from the pre-outburst phase available, while the IRS spectrum, PACS, and APEX data are from the post-outburst period. Thus, its SED does not represent an actual state of the object, and the derived $T_{\text {bol }}$ and $L_{\text {bol }}$ values are unreliable. Pre- and post-outburst SEDs and model fits for this protostar can be found in Fischer et al. (2012). HOPS 223 is the only protostar with an SED affected by extreme variability. A few more protostars, HOPS 71, 132, $143,228,274$, and 299, show notable discrepancy between the IRAC and IRS fluxes, and to a minor extent between MIPS $24 \mu \mathrm{m}$ and IRS, and thus have somewhat unreliable SEDs and SED-derived parameters. HOPS 383, which was identified as an outbursting Class 0 protostar by Safron et al. (2015), does not appear variable in the SED presented here, since we adopted post-outburst IRAC 3.6 and $4.5 \mu$ m fluxes obtained by the YSOVAR program (Morales-Calderón et al. 2011; Rebull et al. 2014) to construct a representative post-outburst SED for this object.

\subsection{Determination of $L_{b o l}, T_{b o l}$, Spectral Index, and SED Classification}

The SEDs provide the means to determine $L_{\mathrm{bol}}, T_{\mathrm{bol}}$, and the $4.5-24 \mu \mathrm{m}$ spectral indices for our sample of protostars. For measuring the near- to mid-IR SED slope $\left(n=d \log \left(\lambda F_{\lambda}\right) / d \log (\lambda)\right)$, we chose a spectral index between 4.5 and $24 \mu \mathrm{m}$ to minimize the effect of extinction on the shortwavelength data point; also, the IRAC $4.5 \mu \mathrm{m}$ fluxes for our HOPS targets are more complete than the IRAC $3.6 \mu$ m fluxes owing to the lower extinction at this wavelength. For calculating $L_{\mathrm{bol}}$ and $T_{\mathrm{bol}}$, we used all available fluxes for each object, including the IRS spectrum, assumed a distance of $420 \mathrm{pc}$, and used trapezoidal summation; for $T_{\text {bol }}$, we applied the equation from Myers \& Ladd (1993). Figure 2 shows the distribution of $n_{4.5-24}, T_{\mathrm{bol}}$, and $L_{\mathrm{bol}}$ values for our targets. There is a peak in the distribution of spectral indices around 0 , while the distribution of $T_{\text {bol }}$ values is relatively uniform from about 30 to $800 \mathrm{~K}$. The bolometric luminosities cover a wide range, with a broad peak around $1 L_{\odot}$. The median $L_{\text {bol }}, T_{\text {bol }}$, and $n_{4.5-24}$ values are $1.1 L_{\odot}, 146 \mathrm{~K}$, and 0.68 , respectively.

Our distribution of $L_{\mathrm{bol}}$ values is very similar to the observed luminosity function of Orion protostars presented in Kryukova et al. (2012); both distributions peak around $1 L_{\odot}$ and include 

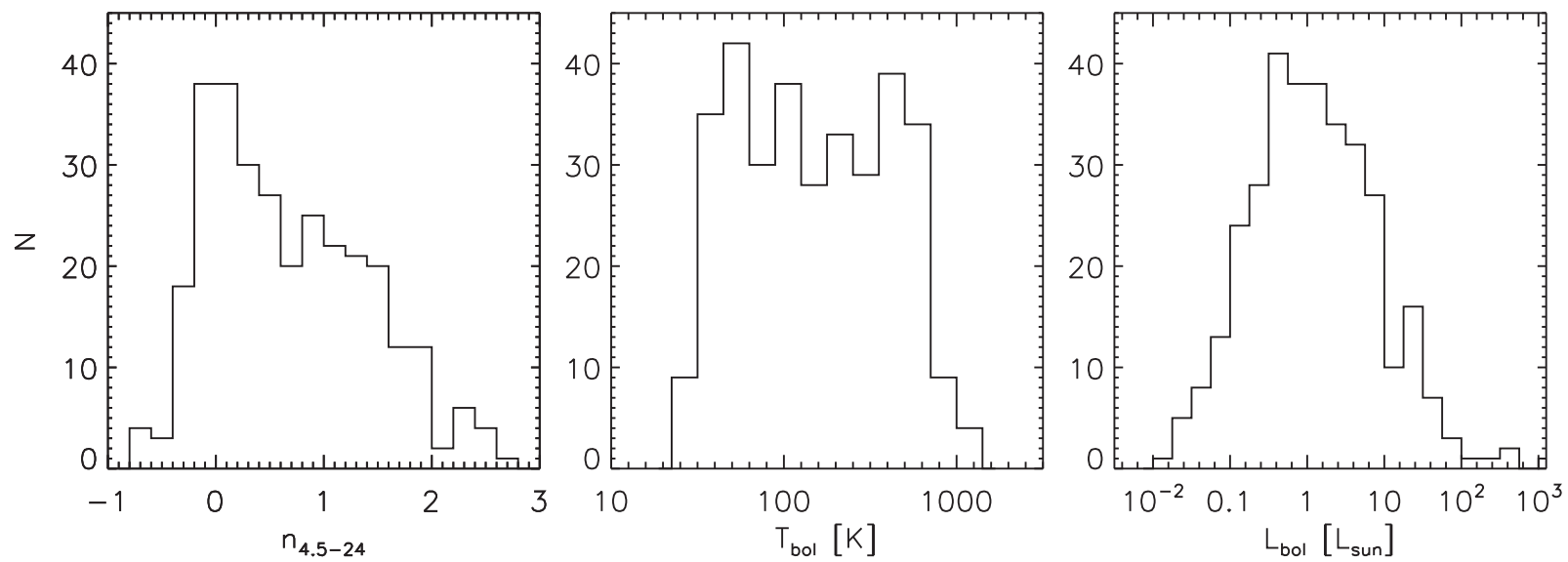

Figure 2. Histograms of the 4.5-24 $\mu \mathrm{m}$ spectral indices (left), bolometric temperatures (middle), and bolometric luminosities (right) for the $330 \mathrm{YSOs}$ in our sample.

values from $\sim 0.02 L_{\odot}$ up to several hundred $L_{\odot}$. Some differences between the two distributions are expected, given that Kryukova et al. (2012) only had Spitzer 3.6-24 $\mu \mathrm{m}$ data available and thus had to extrapolate $L_{\mathrm{bol}}$ from the measured near- to mid-infrared luminosity. The main difference is a somewhat larger number of protostars with $L_{\mathrm{bol}} \lesssim 0.5 L_{\odot}$ for the Kryukova et al. (2012) Orion sample; our median $L_{\text {bol }}$ value amounts to $1.1 L_{\odot}$, while their value is $0.8 L_{\odot}$. However, with the contaminating sources removed from their sample (which tend to have lower luminosities; see Kryukova et al. 2012 for details), their median bolometric luminosity and our value match. Overall, given that Orion is considered a region of highmass star formation, its luminosity function is similar to that of other regions where massive star forms (Kryukova et al. 2012, 2014), and it is different from that of low-mass star-forming regions such as Taurus and Ophiuchus (Kryukova et al. 2012; Dunham et al. 2013). Compared to the sample of 230 protostars in 18 different molecular clouds studied by Dunham et al. (2013), the observed (i.e., not extinctioncorrected) $L_{\text {bol }}$ values of those protostars span from 0.01 to $69 L_{\odot}$, with a median value of $0.9 L_{\odot}$. However, given that almost half the protostars in the Dunham et al. (2013) sample lack far-IR and submillimeter data, the true luminosities are likely higher, which would bring the median closer to the Orion value. Finally, we note that the distribution of observed $T_{\text {bol }}$ values from Dunham et al. (2013) is similar to our distribution for Orion protostars; the median $T_{\mathrm{bol}}$ of their and our sample is 160 and $146 \mathrm{~K}$, respectively, and the bulk of their protostars also has $T_{\text {bol }}$ values between 30 and $1000 \mathrm{~K}$, with a tail down to temperatures of $\sim 10 \mathrm{~K}$ and another tail up to $T_{\mathrm{bol}}=2700 \mathrm{~K}$.

To separate our targets into Class 0, Class I, Class II, and flat-spectrum sources, we used the $4.5-24 \mu \mathrm{m}$ spectral index $\left(n_{4.5-24}\right)$ and/or bolometric temperature $\left(T_{\text {bol }}\right)$ : Class 0 protostars have $n_{4.5-24}>0.3$ and $T_{\text {bol }}<70 \mathrm{~K}$, Class I protostars have $n_{4.5-24}>0.3$ and $T_{\text {bol }}>70 \mathrm{~K}$, flat-spectrum sources have $-0.3<n_{4.5-24}<0.3$, and Class II pre-main-sequence stars have $n_{4.5-24}<-0.3$. Based on this, we identify 92 targets as Class 0 protostars, 125 as Class I protostars, 102 as flatspectrum sources, and 11 as Class II pre-main-sequence stars (see Table 1 and Figure 3). There are nine protostars with $T_{\text {bol }}$ values between 66.5 and $73.5 \mathrm{~K}$ (which corresponds to a $\pm 5 \%$ range around the Class 0-I boundary of $70 \mathrm{~K}$ ); six of them have $T_{\mathrm{bol}}>70 \mathrm{~K}$ (HOPS $1,18,186,256,322,370$ ), and the other three have $T_{\text {bol }}$ values just below $70 \mathrm{~K}$ (HOPS 75, 250, 361). These protostars' classification is less firm than for the

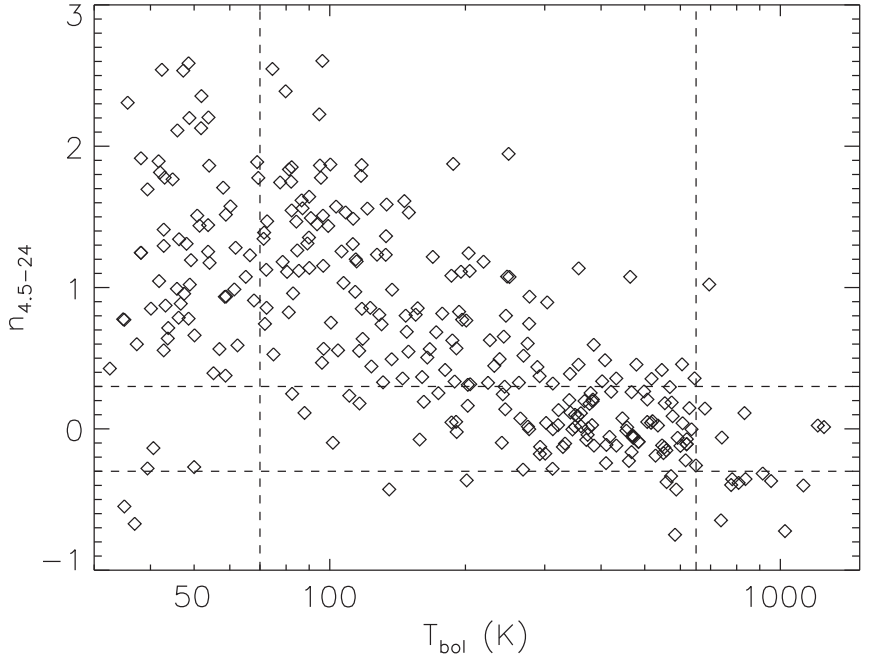

Figure 3. The 4.5-24 $\mu \mathrm{m}$ spectral index vs. the bolometric temperature for the 330 YSOs in our sample. The dashed lines delineate the regions that define the various SED classes (see text for details).

other HOPS targets. There are also a few flat-spectrum sources whose classification is more uncertain: HOPS $45,183,192$, 194, 210, 264, and 281 should be Class I protostars based on their 4.5-24 $\mu \mathrm{m}$ spectral index, but when considering the IRS spectrum (specifically, the 5-25 $\mu \mathrm{m}$ spectral index), they fall into the flat-spectrum regime $\left(n_{5-25}<0.3\right)$. Also, for HOPS 45 and 194 the $T_{\text {bol }}$ values are relatively high $(>500 \mathrm{~K})$. Similarly, HOPS 33, 134, 242, 255, and 284 should be Class II pre-mainsequence stars based on their 4.5-24 $\mu \mathrm{m}$ spectral index, but the spectral slope over the IRS wavelength range suggests that they are flat-spectrum sources. In these cases where the $n_{4.5-24}$ and $n_{5-25}$ spectral indices were somewhat discrepant, we adopted the latter, and thus these objects were classified as flat-spectrum sources.

There are five objects with $T_{\text {bol }}<70 \mathrm{~K}$ and $n_{4.5-24}<0$ (HOPS 164, 340, 341, 373, 405); despite their negative 4.5-24 $\mu \mathrm{m}$ SED slopes, their SEDs either show or imply a deep silicate absorption feature at $10 \mu \mathrm{m}$, rise steeply in the mid- to far-IR, and their long-wavelength emission is strong. Thus, their $T_{\text {bol }}$ values are low, and we identify them as Class 0 protostars, even though they have $4.5-24 \mu \mathrm{m}$ spectral indices not typical of embedded protostars. In particular, HOPS 341, 373 , and 405 are likely young protostars with dense envelopes (Stutz et al. 2013; see also Section 7.2.1). In the case of HOPS 
373 , the $4.5 \mu \mathrm{m}$ flux may be contaminated by bright $\mathrm{H}_{2}$ emission from an outflow shock, rendering the $n_{4.5-24}$ value more unreliable. This might also explain the negative 4.5-24 $\mu \mathrm{m}$ spectral index for the other four protostars.

Finally, the few Class II objects in our sample were thought to be potential protostars prior to their observations with Herschel. Their 4.5-24 $\mu \mathrm{m}$ SED slopes are usually just slightly more negative than the cutoff for a flat-spectrum source $(-0.3)$; three Class II pre-main-sequence stars (HOPS 22, 184, 201) have SEDs that are typical of disks with inner holes, displaying a $10 \mu \mathrm{m}$ silicate emission feature and a rising SED from 12 to about $20 \mu \mathrm{m}$ (e.g., Kim et al. 2013). The SEDs of the other Class II objects are similar to those of flat-spectrum sources; thus, they could have (remnant) envelopes that contribute to their long-wavelength emission.

Our HOPS sample is mostly complete in the number of Class 0 , Class I, and flat-spectrum sources in the areas of Orion surveyed by Spitzer, excluding the Orion Nebula (see Megeath et al. 2012; Stutz et al. 2013). Of the 357 unique YSOs originally identified in Spitzer data that were included in the HOPS sample and observed with PACS, 322 were detected at least at $70 \mu \mathrm{m}$, which amounts to a fraction of $90 \%$. We removed likely contaminants and added 16 new protostars discovered in PACS data to get to our sample of 330 YSOs, most of which are protostars. Our lowest $L_{\text {bol }}$ source is HOPS 208 , with $L_{\mathrm{bol}}=0.017 L_{\odot}$. This protostar also has the lowest PACS $70 \mu \mathrm{m}$ flux in our sample $(8.2 \mathrm{mJy})$. Overall, our sample has 27 protostars with $L_{\mathrm{bol}}<0.1 L_{\odot}$, which places them in the luminosity range of very low luminosity objects (VeLLOs; di Francesco et al. 2007; Dunham et al. 2008). The number of VeLLOs in our sample is likely larger, given that VeLLOs are defined as having internal luminosities less than $0.1 L_{\odot}$, and the bolometric luminosity has contributions from both the internal luminosity and that due to external heating (see Dunham et al. 2008). In addition, our sample could miss fainter flat-spectrum sources and Class 0 and Class I protostars. In fact, there are several faint YSOs without PACS data that were excluded from our sample, but do have Spitzer detections (see Appendix C.2.1).

\section{MODEL GRID}

To characterize the SEDs of our HOPS sample in a uniform manner, we fit the data to simple but physically plausible models. In this way we can assess how well such simple models can fit the data, and how the quality of the fits changes with evolutionary class. We can also determine the full range of physical parameters implied by the fits and the range of parameters for each protostellar class. There are degeneracies and biases in the fits, and the uncertainties in model parameters will vary from object to object, but our results represent a first step in estimating physical parameters that describe the protostars in our sample.

We use a large model grid calculated using the 2008 version of the Whitney et al. (2003b, 2003a) Monte Carlo radiative transfer code (see Stutz et al. 2013); an early version of the grid was presented in Ali et al. (2010). Each model consists of a central protostar, a circumstellar disk, and an envelope; the radiation released by the star and the accretion is reprocessed by the disk and envelope. The density in the disk is described by power laws in the radial and vertical directions, while the density distribution in the envelope corresponds to that of a rotating, collapsing cloud core with constant infall rate (the so-

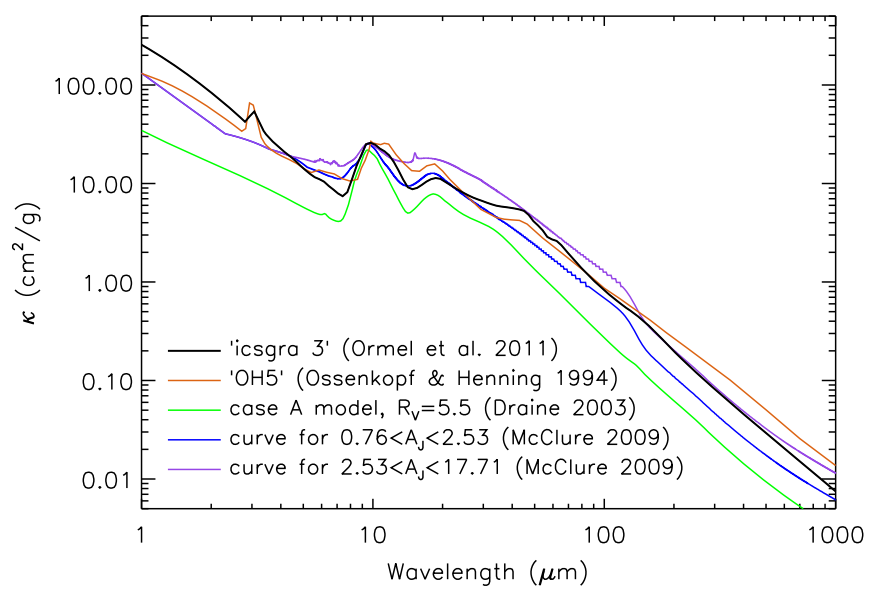

Figure 4. Extinction opacities of the Ormel et al. (2011) dust model "icsgra3" (black) compared to other dust opacities from the literature: grains with thin ice mantles after $10^{5} \mathrm{yr}$ of coagulation with a gas density of $10^{6} \mathrm{~cm}^{-3}$ from Ossenkopf \& Henning (1994) (orange); case A model of carbon and silicate dust for $R_{V}=5.5$ from Draine (2003) (green); and two extinction curves derived for star-forming regions by McClure (2009), one for $0.76<A_{J}<2.53$ (blue), and one for $2.53<A_{J}<17.71$ (purple).

called TSC model, after Terebey et al. 1984; see also Ulrich 1976; Cassen \& Moosman 1981). The envelope also contains an outflow cavity, whose walls are assumed to follow a polynomial shape. At favorable inclination angles, this evacuated cavity allows radiation from the inner envelope and disk regions to reach the observer directly. Also, radiation is scattered off the cavity walls and can increase the near-IR emission from a protostellar system.

We used dust opacities from Ormel et al. (2011) to account for larger, icy grains (as opposed to the small grains made of amorphous silicates typically found in the interstellar medium). We adopted their dust model that includes graphite grains without ice coating and ice-coated silicates, with a size distribution that assumes growth of aggregates for $3 \times 10^{5}$ yr, when grains have grown up to $3 \mu \mathrm{m}$ in size ("icsgra3"). Particle sizes range from 0.1 to $3 \mu \mathrm{m}$, with a number density that is roughly proportional to $a^{-2.3}$ (where $a$ is the particle radius). Figure 4 shows our adopted opacities compared to different ones found in the literature. The opacities from Draine (2003) assume a mixture of small carbonaceous and amorphous silicate grains. Including larger and icy grains broadens the $10 \mu \mathrm{m}$ silicate feature (which is mostly due to the libration mode of water ice) and causes additional absorption at $3 \mu \mathrm{m}$ and in the 40-60 $\mu \mathrm{m}$ range (all mostly due to the presence of water ice). The mid-IR opacities of the "icsgra3" dust model are similar to the ones determined by McClure (2009) for starforming regions and also to those used by Tobin et al. (2008) to model an edge-on Class 0 protostar; in the mid- to far-IR, they resemble the opacities of Ossenkopf \& Henning (1994), which are often used to model embedded sources. In Figure 4, we show model "OH5" from Ossenkopf \& Henning (1994), which is listed as the fifth model in their Table 1 and corresponds to grains with thin ice mantles after $10^{5} \mathrm{yr}$ of coagulation and a gas density of $10^{6} \mathrm{~cm}^{-3}$. We could not use the "OH5" opacities for our model grid, since that opacity law does not include scattering properties (which are required by the Whitney Monte Carlo radiative transfer code). Other authors have modified the "OH5" dust to include the scattering cross section and extend the opacities to shorter and longer wavelengths (Young \& Evans 2005; Dunham et al. 2010). 
Table 3

Model Parameters

\begin{tabular}{|c|c|c|c|}
\hline Parameter & Description & Values & Units \\
\hline$M_{*}$ & Stellar mass & 0.5 & $M_{\odot}$ \\
\hline$T_{*}^{*}$ & Stellar effective temperature & 4000 & $\mathrm{~K}$ \\
\hline \multicolumn{4}{|c|}{ Disk Properties } \\
\hline$M_{\text {disk }}$ & Disk mass & 0.05 & $M_{\odot}$ \\
\hline$B$ & Vertical exponent in disk density law & 1.25 & $\ldots$ \\
\hline$\dot{M}_{\text {disk, } 1}$ & Disk-to-star accretion rate for $R_{\text {star }}=0.67 R_{\odot}$ & $0,1.14 \times 10^{-8}, 5.17 \times 10^{-8}$ & $M_{\odot} \mathrm{yr}^{-1}$ \\
\hline$\dot{M}_{\text {disk }, 2}$ & Disk-to-star accretion rate for $R_{\text {star }}=2.09 R_{\odot}$ & $3.67 \times 10^{-7}, 1.63 \times 10^{-6}$ & $M_{\odot} \mathrm{yr}^{-1}$ \\
\hline$\dot{M}_{\text {disk }, 3}$ & Disk-to-star accretion rate for $R_{\text {star }}=6.61 R_{\odot}$ & $1.14 \times 10^{-5}, 5.15 \times 10^{-5}, 1.66 \times 10^{-4}$ & $M_{\odot} \mathrm{yr}^{-1}$ \\
\hline$R_{\text {trunc }}$ & Magnetospheric truncation radius ${ }^{\mathrm{a}}$ & 3 & $R_{*}$ \\
\hline$f_{\text {spot }}$ & Fractional area of the hot spots on the star ${ }^{\mathrm{b}}$ & 0.01 & $\cdots$ \\
\hline & & $5.95 \times 10^{-20}, 1.19 \times 10^{-19}, 1.78 \times 10^{-19}$ & \\
\hline & & $2.38 \times 10^{-19}, 5.95 \times 10^{-19}, 1.19 \times 10^{-18}$ & \\
\hline & & $1.78 \times 10^{-18}, 2.38 \times 10^{-18}, 5.95 \times 10^{-18}$ & \\
\hline & & $1.19 \times 10^{-17}, 1.78 \times 10^{-17}, 2.38 \times 10^{-17}$ & \\
\hline & & $5.95 \times 10^{-17}, 1.19 \times 10^{-16}, 1.78 \times 10^{-16}$ & \\
\hline$R_{c}$ & Centrifugal radius of TSC envelope & $=R_{\mathrm{disk}}$ & $\mathrm{au}$ \\
\hline$\theta$ & Cavity opening angle & $5,15,25,35,45$ & degrees \\
\hline$b_{\text {cav }}$ & Exponent for cavity shape ${ }^{\mathrm{e}}$ (polynomial) & 1.5 & $\ldots$ \\
\hline$z_{\text {cav }}$ & Vertical offset of cavity wall & 0 & au \\
\hline \multicolumn{4}{|c|}{ Derived Parameters } \\
\hline$L_{*}$ & Stellar luminosity ${ }^{\mathrm{f}}$ & $0.1,1,10$ & $L_{\odot}$ \\
\hline$L_{\mathrm{tot}}$ & Total luminosity $(\text { star }+ \text { accretion })^{\mathrm{g}}$ & $0.1,0.3,1.0,3.1,10.1,30.2,101,303$ & $L_{\odot}$ \\
\hline
\end{tabular}

Notes. The dust opacities used for these models are those called "icsgra3" from Ormel et al. (2011).

a This radius applies to the gas. The inner disk radius for the dust is equal to the dust destruction radius. The scale height of the disk at the dust sublimation radius is set to the hydrostatic equilibrium solution.

${ }^{b}$ The hot spots are caused by the accretion columns that reach from the magnetospheric truncation radius to the star.

${ }^{c}$ The inner envelope radius is set to the dust destruction radius.

$\mathrm{d}$ The actual input parameter for the Whitney code is the envelope infall rate, which can be derived from $\rho_{1000}$ using Equation (2). The first six $\rho_{1000}$ values correspond to envelope infall rates of $0,5.0 \times 10^{-8}, 7.5 \times 10^{-8}, 1.0 \times 10^{-7}, 2.5 \times 10^{-7}$, and $5.0 \times 10^{-7} M_{\odot} \mathrm{yr}^{-1}$; the other values can be similarly deduced.

e The cavity walls are assumed to have a polynomial shape; no material is assumed to lie inside the cavity. Also, the ambient density (outside the envelope) is 0 .

${ }^{\mathrm{f}}$ The three values of $L_{*}$ correspond to the three different stellar radii.

g The total luminosities combine the stellar luminosities and the accretion luminosities (which depend on $\dot{M}_{\text {disk }}$ ).

${ }^{\mathrm{h}}$ For each model, the emitted fluxes are calculated for 24 apertures ranging from 420 to 10,080 au, in steps of 420 au.

\subsection{Model Parameters}

There are 3040 models in the grid; they cover eight values for the total (i.e., intrinsic) luminosity, four disk radii, 19 envelope infall rates (which correspond to envelope densities), and five cavity opening angles. Each model is calculated for 10 different inclination angles, from $18^{\circ} .2$ to $87^{\circ} .2$, in equal steps in $\cos (i)$ (starting at 0.95 and ending at 0.05 ), resulting in 30,400 different model SEDs. The values for the various model parameters are listed in Table 3. Since there are a large number of parameters that can be set in the Whitney radiative transfer models, we focused on varying those parameters that affect the
SED the most, leaving the other parameters at some typical values. For example, we assumed a stellar mass of $0.5 M_{\odot}$, a disk mass of $0.05 M_{\odot}$, and an envelope outer radius of $10,000 \mathrm{au}$. The stellar mass enters the code in two ways. First, it is needed to relate the density of the envelope to the infall rate (see Equation (1) below). Since we fit the density of the envelope, the infall rate plays no role in the best-fit envelope parameters; any stellar mass can be chosen to determine the infall rate for a given best-fit envelope density. Second, the stellar mass is combined with the stellar radius and disk accretion rate to set the disk accretion luminosity. Given that the accretion luminosity is the actual parameter that influences 
the SED, it does not matter which of the three factors is varied. For simplicity and reasons described below, we varied the disk accretion rate and the stellar radius, but left the stellar mass constant, to achieve different values for this component of the luminosity.

The total luminosity for each system consists of the stellar luminosity (derived from a $4000 \mathrm{~K}$ stellar atmosphere model), the accretion luminosity resulting from material accreting through the disk down to the disk truncation radius, and the accretion luminosity from the hot spots on the stellar surface, where the accretion columns, which start at the magnetospheric truncation radius, land (these columns are not included in the modeled density distribution, since they do not contain dust and do not have a source of opacity in the radiative transfer models). Typically, the accretion luminosity from the hot spots is much larger than the disk accretion luminosity; in our models, the former is about a factor of 9 larger than the latter. We chose three different stellar radii, $0.67,2.09$, and $6.61 R_{\odot}$ (with the same stellar temperature), resulting in three different stellar luminosities. Since both components of the accretion luminosity depend on the disk accretion rate, choosing a total of eight different disk accretion rates (three for the $0.67 R_{\odot}$ star, two for the $2.09 R_{\odot}$ star, and three for the $6.61 R_{\odot}$ star) results in eight values for the total luminosity used in the grid (see Table 3). The input spectrum produced by the central protostar depends on the relative contributions from the intrinsic stellar luminosity (which peaks at $0.7 \mu \mathrm{m}$ ) and the accretion luminosity (which is radiated primarily in the UV). In the models, it can be altered to some degree by choosing different combinations of the disk accretion rate and stellar radius (the former affects only the accretion luminosity, while the latter affects both the stellar and accretion luminosity). However, the effect of the input spectrum on the output SED is negligible. Consequently, we cannot reliably measure the relative contributions of stellar and accretion luminosity through our SED fits. Instead, we adjusted the particular values for the stellar radius and disk accretion rate to set the values of the total luminosity.

For our model grid, we chose four values for the disk outer radius, which we set equal to the centrifugal radius $\left(R_{c}\right)$. In a TSC model, the centrifugal radius is the position in the disk where material falling in from the envelope accumulates; owing to envelope rotation, material from the envelope's equatorial plane lands at $R_{c}$, while material from higher latitudes falls closer to the star. The disk could extend beyond $R_{c}$, but in our models it ends at $R_{c}$. In this work, we use the terms "disk (outer) radius" and "centrifugal radius" interchangeably. The primary effect of $R_{c}$ is to set the rotation rate of the infalling gas and thereby determine the density structure of the envelope (Kenyon et al. 1993).

The largest number of parameter values in our grid is for the envelope infall rate. The envelope infall rate used as an input in the Whitney code sets the density of the envelope for a given mass of the protostar. Since the SED depends on the density of the envelope (and not directly on the infall rate, which is only inferred from the density and the acceleration due to gravity from the central protostar), in this work we report a reference envelope density instead of the envelope infall rate as one of our model parameters. For the TSC model, the envelope infall rate $\dot{M}_{\text {env }}$ and the reference density at 1 au in the limit of no rotation $\left(R_{c}=0\right)$ are related as follows (see Kenyon et al.
1993):

$$
\rho_{1}=5.318 \times 10^{-14}\left(\frac{\dot{M}_{\mathrm{env}}}{10^{-5} M_{\odot} \mathrm{yr}^{-1}}\right)\left(\frac{M_{*}}{1 M_{\odot}}\right)^{-1 / 2} \mathrm{~g} \mathrm{~cm}^{-3},
$$

where $M_{*}$ is the mass of the central protostar, which is assumed to be $0.5 M_{\odot}$ in our model grid. The density distribution in the envelope follows a power law, $\rho \propto r^{-3 / 2}$, at radii larger than the centrifugal radius, $R_{c}$, but then flattens as a result of the rotation of the envelope. The density reported by $\rho_{1}$ assumes a spherically symmetric envelope with a $-3 / 2$ power-law exponent valid down to the smallest radii, and it is higher than the angle-averaged density of a rotating envelope at $1 \mathrm{au}$. To quote densities that are closer to actual values found in the modeled rotating envelopes (which have $R_{c}$ values ranging from 5 to $500 \mathrm{au}$ ), we report $\rho_{1000}$, the density at $1000 \mathrm{au}$ for a $\rho \propto r^{-3 / 2}$ envelope with a $0.5 M_{\odot}$ protostar:

$$
\begin{aligned}
\rho_{1000} & =\rho_{1}\left(\frac{1}{1000}\right)^{3 / 2} \\
& =2.378 \times 10^{-18}\left(\frac{\dot{M}_{\mathrm{env}}}{10^{-5} M_{\odot} \mathrm{yr}^{-1}}\right) \mathrm{g} \mathrm{cm}^{-3} .
\end{aligned}
$$

Thus, the range of reference densities probed in our model grid, from $1.2 \times 10^{-20}$ to $1.8 \times 10^{-16} \mathrm{~g} \mathrm{~cm}^{-3}$ (see Table 3 ), would correspond to envelope infall rates from $5.0 \times 10^{-8}$ to $7.5 \times 10^{-4} M_{\odot} \mathrm{yr}^{-1}$, assuming $M_{*}=0.5 M_{\odot}$ (this does not account for a reduction of the infalling mass due to clearing by outflow cavities). In Figure 5, we show the radial density profiles for two TSC models with 5 and 500 au centrifugal radii. The density profiles are azimuthally symmetric and show the flattening of the density distribution inside $R_{c}$ due to envelope rotation. These plots demonstrate that the density $\rho_{1}$ is much higher than the angle-averaged density at $1 \mathrm{au} ; \rho_{1000}$ seems to yield more physical values for the density in the envelope at $1000 \mathrm{au}$, even for $R_{c}$ values of $500 \mathrm{au}$.

As can be seen from the values of the envelope density in Table 3, there is one set of models with an envelope density of 0 . These are models that do not contain an envelope component; the entire excess emission is caused by the circumstellar disk. If an object is best fit by such a model, it would indicate that it is more evolved, having already dispersed its envelope.

The cavities in our models range from $5^{\circ}$ to $45^{\circ}$ and are defined such that $z \propto \tilde{r}^{1.5}$, where $\tilde{r}$ and $z$ are the cylindrical coordinates for the radial and vertical direction, respectively, and $\tilde{r}_{\max }=z_{\max } \tan \theta$, with $\theta$ defined as the cavity opening angle that is specified in the parameter file of the Whitney radiative transfer code. In this code, $z_{\max }$ is set to the envelope outer radius. Thus, a polynomial-shaped cavity, which is wider at smaller $\tilde{r}$ values and then converges toward the specified opening angle, is somewhat larger than this opening angle at the outer envelope radius (see Figure 6). This effect is most noticeable at larger cavity opening angles, but negligible for small cavities. A different definition of the cavity size, where $\tilde{r}_{\mathrm{max}}=R_{\mathrm{env}} \sin \theta$ and $z_{\max }=R_{\mathrm{env}} \cos \theta$ (with $R_{\mathrm{env}}$ as the envelope outer radius), results in $z$ values that are a factor of $1 / \cos \theta$ larger, and thus the cavity reaches the specified opening angle at the outer envelope radius. For this work, the adopted definition of the cavity opening angle is 

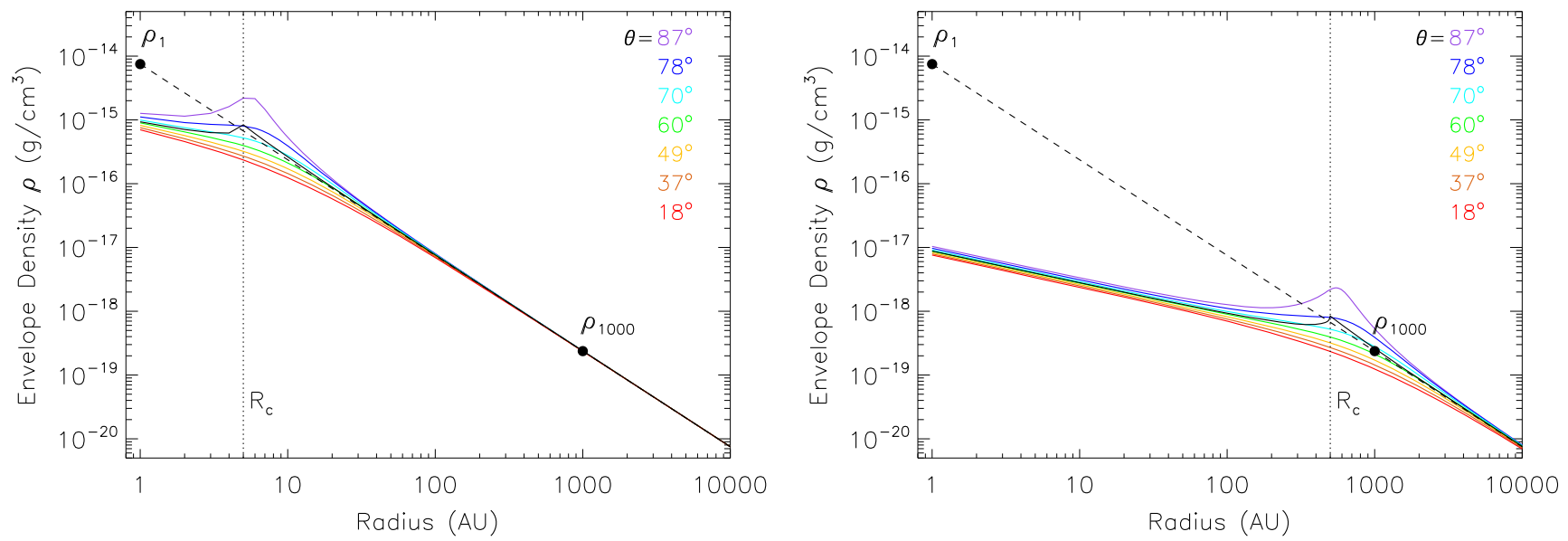

Figure 5. Envelope density vs. radius for a model protostar with $\dot{M}_{\text {env }}=1.0 \times 10^{-6} M_{\odot} \mathrm{yr}^{-1}, M_{*}=0.5 M_{\odot}$, and $R_{c}=5$ au (left) and 500 au (right) to show the difference between the reference densities $\rho_{1}$ and $\rho_{1000}$. The lines with different colors represent radial density profiles for different polar angles $\theta$; the black line represents the angle-averaged density profile (for equations see Adams \& Shu 1986; Whitney et al. 2003b). The dashed line represents an $r^{-3 / 2}$ power law. The vertical dotted line marks the location of the centrifugal radius.

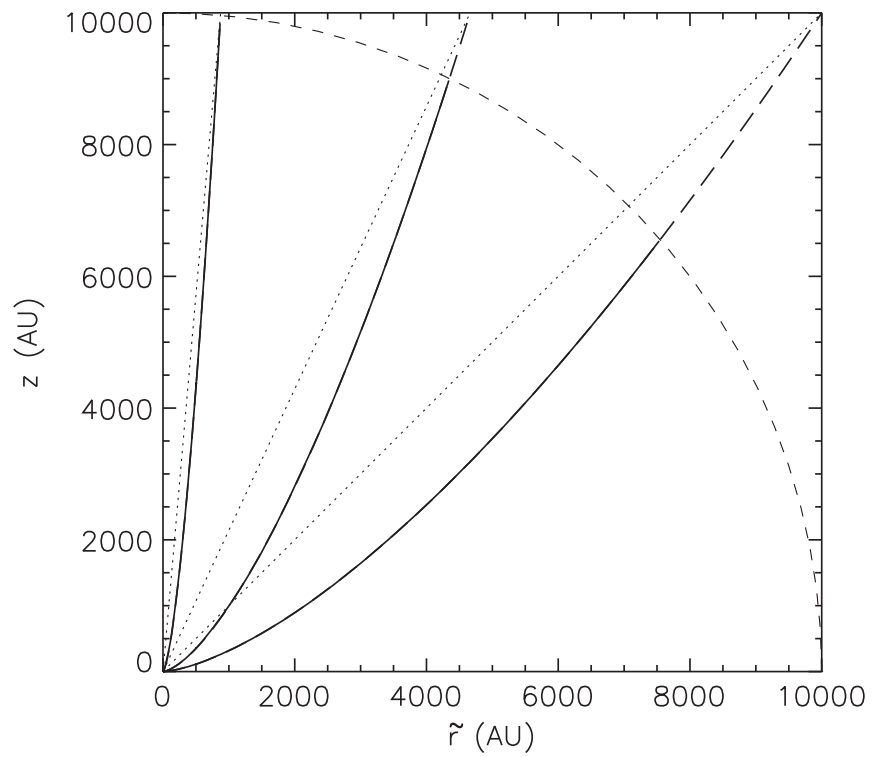

Figure 6. Schematic showing the shape of the cavity assumed in our models for three cavity opening angles $\theta: 5^{\circ}, 25^{\circ}$, and $45^{\circ}$ (from left to right). The cavity walls are defined as a polynomial with exponent $1.5\left(z \propto \tilde{r}^{1.5}\right)$, with $\tilde{r}_{\max }=z_{\max } \tan \theta$, and are shown as solid lines. The outer envelope radius $\left(R_{\text {env }}\right)$ at 10,000 au is shown with a short-dashed line. The dotted lines show a different definition of the cavity size, where $\tilde{r}_{\max }=R_{\text {env }} \sin \theta$ and $z_{\max }=R_{\mathrm{env}} \cos \theta$.

inconsequential, but it becomes relevant when comparing the results of SED modeling to scattered light images that reveal the actual cavity shape and size. We also note that in our models the cavities are evacuated of material, so there is no dust and gas inside the cavity; in reality, there might be some low-density material left that would add to the scattered light (see Fischer et al. 2014).

Figures 7-11 display a few examples of model SEDs from our grid to show the effect of changing those model parameters that influence the resulting SED the most. The inclination angle has a strong effect on the near- and mid-infrared SED (Figure 7). While a low inclination angle results in an overall flat SED in this wavelength region, increasing the inclination angle causes a deeper silicate absorption feature at $10 \mu \mathrm{m}$ and a steep slope beyond it. The far-infrared to millimeter SED is not

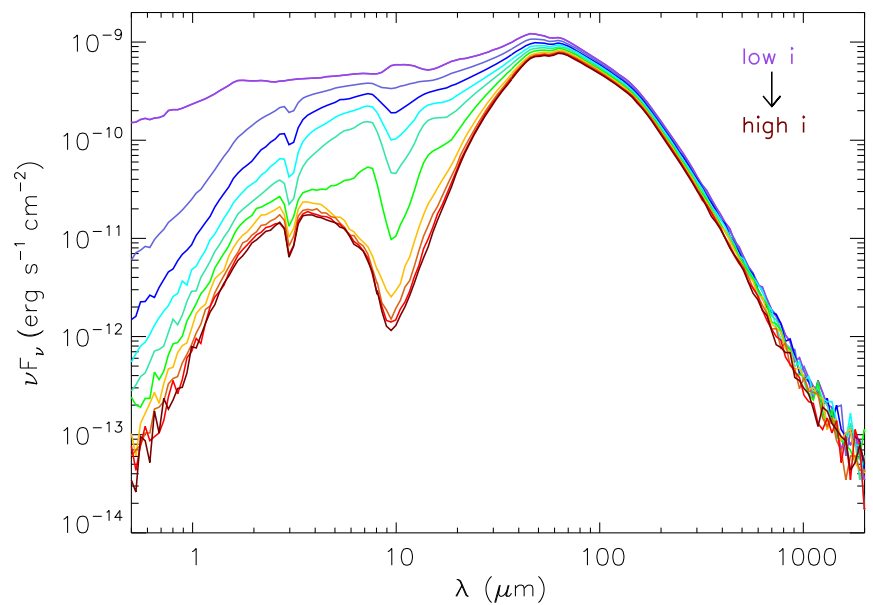

Figure 7. Model from the grid seen at 10 different inclination angles to illustrate the effect of viewing angle on the SED. The model has $L_{\mathrm{tot}}=10.1 L_{\odot}$, $R_{c}=50 \mathrm{au}, \rho_{1000}=1.2 \times 10^{-18} \mathrm{~g} \mathrm{~cm}^{-3}$, and $\theta=15^{\circ}$ and is seen at inclination angles $18^{\circ}, 32^{\circ}, 41^{\circ}, 49^{\circ}, 57^{\circ}, 63^{\circ}, 69^{\circ}, 76^{\circ}, 81^{\circ}$, and $87^{\circ}$ (from top to bottom).

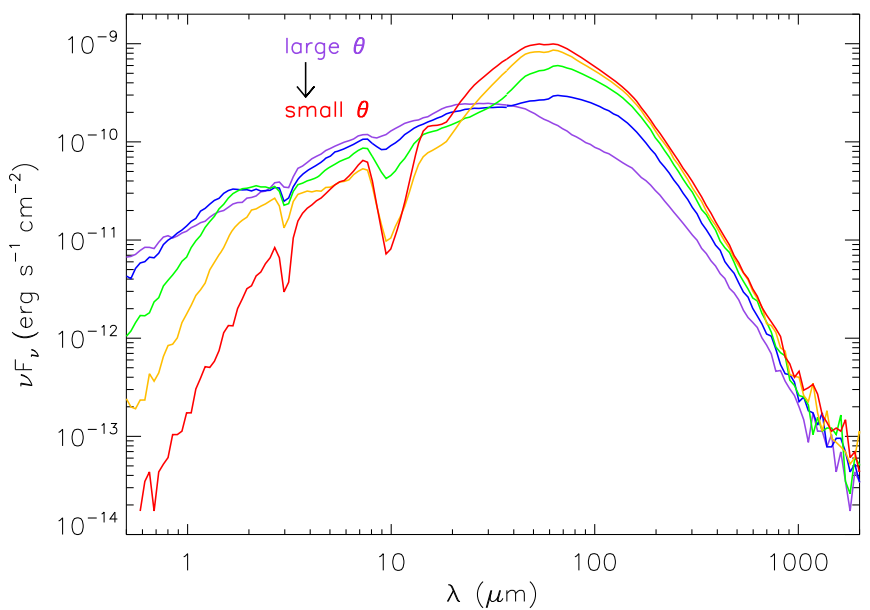

Figure 8. Models from the grid to illustrate the effect of cavity opening angle on the SED. The models have $L_{\mathrm{tot}}=10.1 L_{\odot}, \quad R_{c}=50 \mathrm{au}$, $\rho_{1000}=1.2 \times 10^{-18} \mathrm{~g} \mathrm{~cm}^{-3}, i=63^{\circ}$, but each has a different cavity opening angle: $5^{\circ}$ (red), $15^{\circ}$ (yellow), $25^{\circ}$ (green), $35^{\circ}$ (blue), $45^{\circ}$ (purple). 


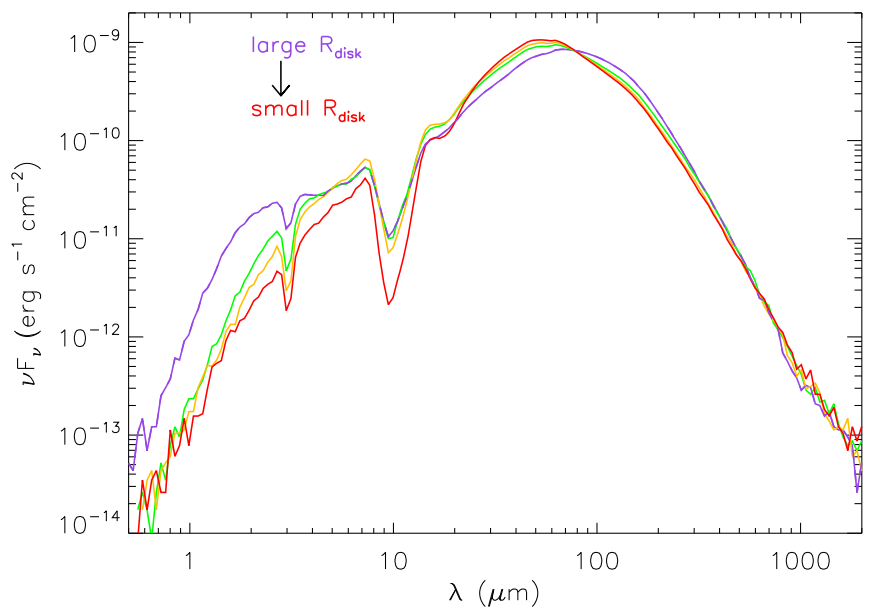

Figure 9. Models from the grid to illustrate the effect of the centrifugal radius $\left(=R_{\text {disk }}\right) \quad$ on the SED. The models have $L_{\text {tot }}=10.1 L_{\odot}$, $\rho_{1000}=1.2 \times 10^{-18} \mathrm{~g} \mathrm{~cm}^{-3}, \theta=5^{\circ}$, and $i=63^{\circ}$, but different disk radii: $5 \mathrm{au}$ (red), $50 \mathrm{au}$ (yellow), $100 \mathrm{au}$ (green), and $500 \mathrm{au}$ (purple).

affected by the inclination angle, since emission at these wavelengths does not suffer from extinction through the envelope.

The cavity opening angle affects the SED shape at all wavelengths (Figure 8). A small cavity only minimally alters the SED compared to a case without a cavity; there is still a deep silicate absorption at $10 \mu \mathrm{m}$ and steep SED slope, but the cavity allows some scattered light to escape in the near-IR. A larger cavity results in higher emission at near- and midinfrared wavelengths and reduced emission in the far-infrared. The effect of the cavity on the SED would change if a different shape for the cavity walls were adopted. For example, cavities where the outer wall follows the streamlines of the infalling gas and dust evacuate less inner envelope material than our polynomial-shaped cavities, resulting in deeper silicate absorption features and steeper mid-infrared SED slopes for the same cavity opening angle (see Furlan et al. 2014). Thus, our cavity opening angles are tied to our assumed cavity shape.

The effect of the centrifugal radius is somewhat similar to those of the cavity opening angle and inclination angle, but less pronounced (Figure 9). Small disk radii imply more slowly rotating, less flattened envelopes and depress the near- and mid-infrared fluxes more than larger disk radii, but even with large disk radii (and more flattened envelopes) there is still sufficient envelope material along the line of sight to cause a pronounced $10 \mu \mathrm{m}$ absorption feature. Overall, our models do not directly constrain the size of the disk; the opacity is dominated by the envelope. Furthermore, the flattening of the envelope that is determined by $R_{c}$ has a similar effect on the SED as changing the outflow cavity opening angle.

Changing the envelope density causes shifts in the SED in terms of both wavelength and flux level: the higher the envelope density, the less flux is emitted at shorter wavelengths, and the more the peak of the SED shifts to longer wavelengths (Figure 10). Deeply embedded protostars have SEDs that peak at $\lambda>100 \mu \mathrm{m}$, steep mid-IR SED slopes, and deep silicate absorption features. The effect of the envelope density on the SED is different from that of the inclination angle, especially in the far-IR: while the SED is not very sensitive to the inclination angle in this wavelength region, the ratio of, e.g., 70 and $160 \mu \mathrm{m}$ fluxes changes considerably depending on the envelope density.

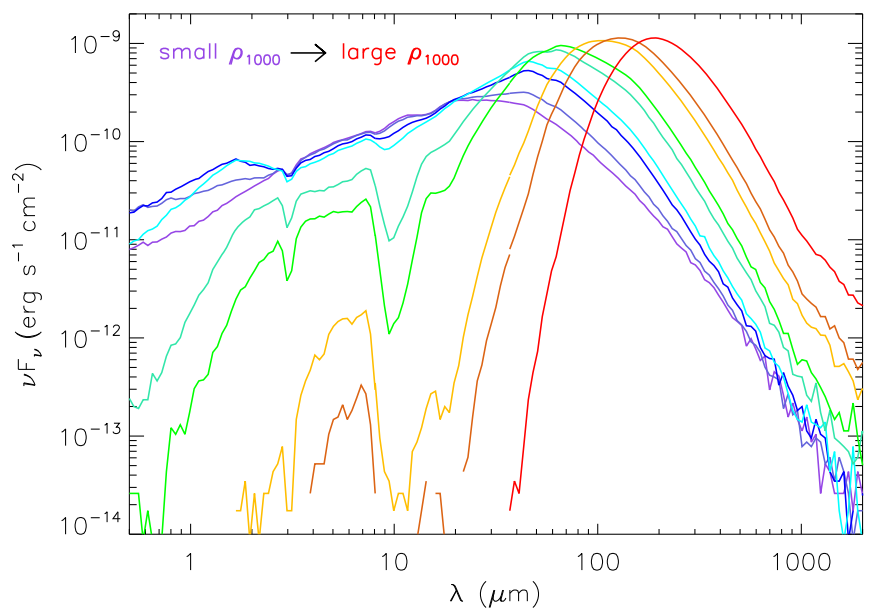

Figure 10. Models from the grid to illustrate the effect of envelope density on the SED. The models have $L_{\mathrm{tot}}=10.1 L_{\odot}, R_{c}=50 \mathrm{au}, \theta=15^{\circ}$, and $i=63^{\circ}$, but different reference densities $\rho_{1000}: 0,2.4 \times 10^{-20}, 1.2 \times 10^{-19}$, $2.4 \times 10^{-19}, 1.2 \times 10^{-18}, 2.4 \times 10^{-18}, 1.2 \times 10^{-17}, 2.4 \times 10^{-17}$, and $1.2 \times 10^{-16} \mathrm{~g} \mathrm{~cm}^{-3}$ (the peak of the SED moves to longer wavelengths as $\rho_{1000}$ increases).

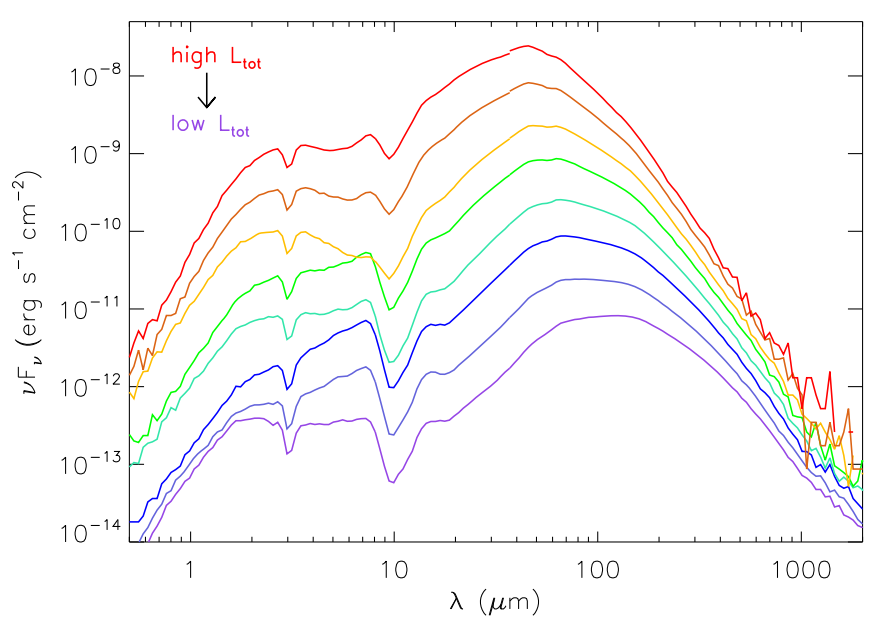

Figure 11. Models from the grid to illustrate the effect of the total luminosity on the SED. The models have $R_{c}=50 \mathrm{au}, \rho_{1000}=1.2 \times 10^{-18} \mathrm{~g} \mathrm{~cm}^{-3}$, $\theta=15^{\circ}$, and $i=63^{\circ}$, but different values for the total luminosity: $0.1,0.3,1.0$, $3.1,10.1,30.2,101$, and $303 L_{\odot}$ (from bottom to top).

The total luminosity of the source has an effect on the overall emission level of the protostar, but does not strongly affect the SED shape. The main effect is that the peak of the SED shifts to longer wavelengths as the luminosity decreases $\left(\lambda_{\text {peak }} \propto L^{-1 / 12}\right.$; Kenyon et al. 1993). Especially when comparing models with $L_{\text {tot }}$ values that differ by a factor of a few, the SED shapes are similar (Figure 11). Thus, one could scale a particular model by a factor between $\sim 0.5$ and 2 and get a good representation of a protostar that is somewhat fainter or brighter, without having to rerun the model calculation with the different input luminosity.

\subsection{Model Apertures}

The model fluxes are computed for 24 different apertures, ranging from 420 to $10,080 \mathrm{au}$ in steps of $420 \mathrm{au}$ (which corresponds to $1^{\prime \prime}$ at the assumed distance of $420 \mathrm{pc}$ to the Orion star-forming complex). For these SED fluxes, no convolution with a PSF is done, and therefore the spatial distribution of the flux is solely due to the extended nature of 


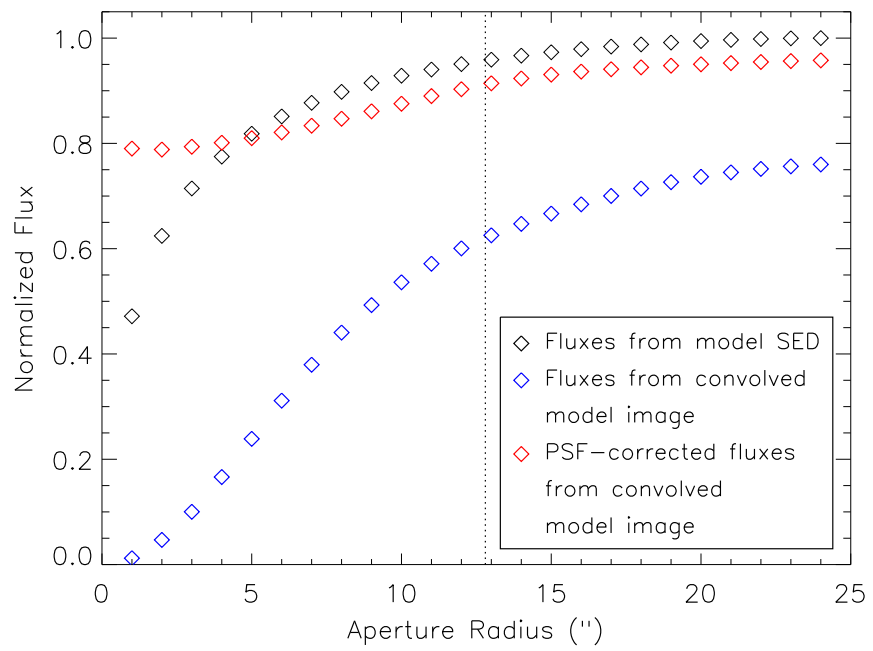

Figure 12. PACS $160 \mu \mathrm{m}$ fluxes vs. aperture radius derived for a model $\left(L_{\mathrm{tot}}=1.0 L_{\odot}, R_{c}=100 \mathrm{au}, \rho_{1000}=2.378 \times 10^{-18} \mathrm{~g} \mathrm{~cm}^{-3}, \quad \theta=15^{\circ}\right.$, $i=63^{\circ}$ ) using different methods. The black symbols represent fluxes from the model SED, the blue symbols fluxes derived using aperture photometry on the model image convolved with the PACS $160 \mu \mathrm{m}$ PSF, and the red symbols fluxes derived from the convolved model image and then corrected for PSF losses (see text for details). The maximum flux from the model SED was used to normalize all other fluxes. The dotted line indicates an aperture radius of 12 !" 8 .

protostars. Since the envelope outer radius is chosen to be $10,000 \mathrm{au}$, the largest aperture encompasses the entire flux emitted by each protostellar system. However, most of the near- and mid-infrared emission comes from smaller spatial scales, so an aperture of about 5000 au will already capture most of the flux emitted at these wavelengths.

For a more accurate comparison of observed and model fluxes, in each infrared photometric band where we have data available, we interpolate model fluxes from the two apertures that bracket the aperture used in measuring the observed fluxes (4" for 2MASS, 2!" 4 for IRAC, PSF photometry for MIPS $24 \mu \mathrm{m}$, with a typical FWHM of 6", 9".6 for PACS 70 and $100 \mu \mathrm{m}, 12$ ". 8 for PACS $160 \mu \mathrm{m})$. For the IRS data points, we use fluxes interpolated for a 5." 3 aperture, since the spectra are composed of two segments, SL (5.2-14 $\mu \mathrm{m}$; slit width of 3 ". 6$)$ and LL (14-38 $\mu \mathrm{m}$, slit width of $10 . " 5)$, and, if any flux mismatches were present, the SL segment was typically scaled to match the LL flux level at $14 \mu \mathrm{m}$ (see, e.g., Furlan et al. 2008). So, fluxes measured in an aperture with a radius of 5!" 3 roughly correspond to fluxes from a 10 ". 6 -wide slit.

Given that our targets are typically extended and that the near- to mid-infrared data have relatively high spatial resolution, measuring fluxes in small apertures (a few arcseconds in radius) will truncate some of the object's flux, so it is important to choose similar apertures for the model fluxes. From about 30 to $100 \mu \mathrm{m}$, the model fluxes calculated for smaller apertures are not very different from the total flux (i.e., the flux from the largest aperture), which is a result of the emission profile in the envelope and the lower spatial resolution at longer wavelengths. To check whether extended source emission in the far-infrared might affect the flux we measure in our models, we calculated a small set of model images at $160 \mu \mathrm{m}$, convolved them with the PACS $160 \mu \mathrm{m}$ PSF, and compared the fluxes from the model images to those written out for the model SEDs (which we refer to as "SED fluxes"; these are the fluxes from the models in the grid). Model images would be the most observationally consistent way to measure

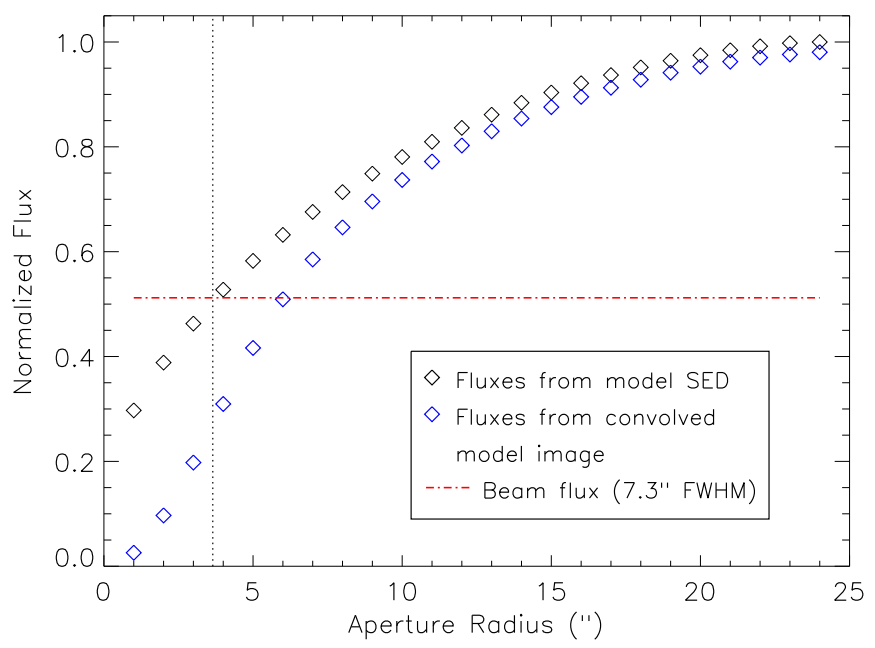

Figure 13. SABOCA $(350 \mu \mathrm{m})$ fluxes vs. aperture radius derived for the same model as in Figure 12 using different methods. The black symbols represent fluxes from the model SED, the blue symbols fluxes derived using aperture photometry on the model image convolved with a Gaussian PSF, and the red dot-dashed line the beam flux (assuming a beam with an FWHM of 7 !' 3 ). The maximum flux from the model SED was used to normalize all other fluxes. The dotted line indicates an aperture radius of 3 !' 65 .

the flux densities, but they are too computationally expensive and would not represent a significant gain.

In Figure 12 we show the fluxes derived for a particular model at $160 \mu \mathrm{m}$ using different methods. The fluxes measured in the convolved model image are lower than the SED fluxes; this is caused by the wide PACS $160 \mu \mathrm{m}$ PSF, which spreads flux to very large radii. Since the shape of the PSF is known, we can correct for these PSF losses (assuming a point source and using standard aperture corrections). The fluxes corrected for these PSF losses are very similar to the SED fluxes, typically within $\sim 5 \%-10 \%$ at apertures larger than $5^{\prime \prime}$. Since our observed fluxes correspond to these PSF-corrected fluxes (we apply aperture corrections to our fluxes measured in a 12!" 8 aperture to account for PSF losses), adopting the SED fluxes from the largest aperture would yield model fluxes that are somewhat too high. Thus, we chose to adopt the SED flux measured in a 12 ". 8 aperture as a good approximation for the model flux we would get if we had model images available for all models in the grid and measured aperture-corrected fluxes in these images. We note that in our PACS data, the $160 \mu \mathrm{m}$ sky annulus, which extends from 12 ". 8 to 25 ". 6 (see B. Ali et al. 2016 , in preparation), can include extended emission from surrounding material and also some envelope emission. In these cases, we often used PSF photometry to minimize contamination from nearby sources and nebulosity; however, PSF fitting was not used for more isolated sources since the envelopes can be marginally resolved at $160 \mu \mathrm{m}$ and thus deviate slightly from the adopted PSF shape.

For the SABOCA and LABOCA data, beam fluxes were adopted; the FWHM of the SABOCA beam is 7!'3, while for the LABOCA beam it is $19^{\prime \prime}$. In order to determine which aperture radius corresponds best to beam fluxes, we created a similar set of model images as above at 350 and $870 \mu \mathrm{m}$, convolved them with Gaussian PSFs, and measured fluxes in the model images using different apertures (see Figures 13 and 14, where we show the results for one model). Fluxes measured in the convolved model image are smaller than the SED fluxes, especially at aperture radii smaller than the FWHM of the beam. We find that the beam fluxes for SABOCA and 


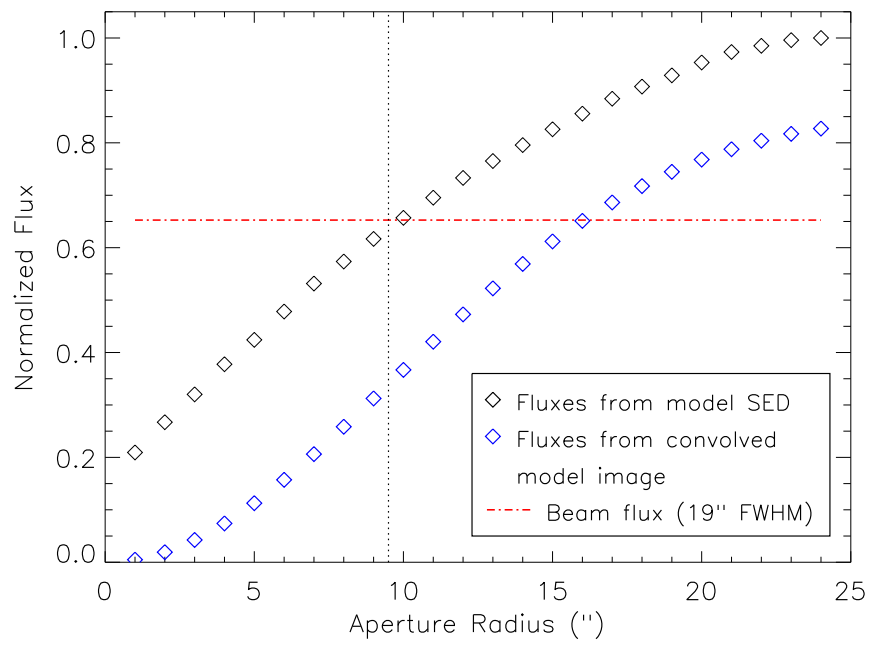

Figure 14. Similar to Figure 13 , but for the LABOCA $(870 \mu \mathrm{m})$ fluxes. The dotted line indicates an aperture radius of 9 ". 5 .

LABOCA are best matched by SED fluxes from apertures with radii half the size of the FWHM of the beam, i.e., 3!" 65 for SABOCA and 9".5 for LABOCA (thus, the aperture sizes are the same as the beam FWHM). This is again an idealized situation, since the measured SABOCA and LABOCA beam fluxes also include extended emission (if the source lies on top of background emission), and thus they could be higher than those from the model.

\subsection{Effect of External Heating}

In our models, the luminosity is determined by the central protostar and the accretion; no external heating is included. The interstellar radiation field (ISRF) could increase the temperature in the outer envelope regions, thus causing an increase in the longer-wavelength fluxes (e.g., Evans et al. 2001; Shirley et al. 2002; Young et al. 2003). It is expected that external heating has a noticeable effect only on low-luminosity sources $\left(\lesssim 1 L_{\odot}\right)$, while objects with strong internal heating are not affected by the ISRF. Moreover, the strength of the ISRF varies spatially (Mathis et al. 1983), and thus its effect on each individual protostar is uncertain. Nonetheless, in the following we estimate the effect of external heating on model fluxes by using a different set of models.

For this model calculation, we used the 2012 version of the Whitney radiative transfer code (Whitney et al. 2013), which allows for the inclusion of external illumination by using the ISRF value in the solar neighborhood from Mathis et al. (1983); to vary the ISRF strength, the adopted value can be scaled by a multiplicative factor and extinguished by a certain amount of foreground extinction. We calculated a small number of models with and without external heating and then compared their far-infrared and submillimeter fluxes. One set of models has $L_{\mathrm{tot}}=0.1 L_{\odot}, R_{c}=100 \mathrm{au}, \theta=15^{\circ}$, and four different reference densities $\rho_{1000}$, ranging from $2.4 \times 10^{-17}$ to $2.4 \times 10^{-20} \mathrm{~g} \mathrm{~cm}^{-3}$. The other set has the same parameters except for $L_{\mathrm{tot}}$, which is $1.0 L_{\odot}$. We calculated models without external heating, with heating from an ISRF equal to that in the solar neighborhood, and with ISRF heating 10 times the solar neighborhood value. For these models, we did not include any foreground extinction for the ISRF; thus, the ISRF heating in these models can be considered an upper limit-especially the 10-fold increase over the ISRF in the solar neighborhood represents an extreme value. Figure 15 shows a few examples of model SEDs with and without external heating. External heating results in flux increases in the far-IR and submillimeter; as expected, it affects low-luminosity sources more, and its effects are also more noticeable for higher-density envelopes.

For a more quantitative comparison of model fluxes in the far-IR and submillimeter, we computed the fluxes for each model in six different bands, those of MIPS $24 \mu \mathrm{m}$, PACS 70, 100 , and $160 \mu \mathrm{m}$, and SABOCA $(350 \mu \mathrm{m})$ and LABOCA $(870 \mu \mathrm{m})$, using apertures as described in Section 4.2. The model fluxes are affected by poorer signal-to-noise ratios at the longest wavelengths, so the $870 \mu \mathrm{m}$ fluxes are less reliable. We subtracted the fluxes of the models without external heating $\left(F_{\text {no.ext.heating }}\right)$ from those with external heating $\left(F_{\text {ext.heating }}\right)$ to determine the flux excess due to external heating. The ratios of these excess fluxes and the model fluxes with external heating $\left(\left(F_{\text {ext.heating }}-F_{\text {no.ext.heating }}\right) / F_{\text {ext.heating }}\right)$ are shown in Figure 16. Given that these ratios depend on the inclination angle to the line of sight, we show them as average values for all 10 inclination angles, as well as the range subtended by all inclination angles. We note overall smaller flux ratios at $350 \mu \mathrm{m}$ owing to the smaller aperture size chosen in this wave band (see Section 4.2).

Our analysis shows that heating by the ISRF results in flux increases in the far-IR and submillimeter that are about a factor of 2-3 higher for envelopes of low-luminosity sources $\left(L_{\text {tot }}=0.1 L_{\odot}\right)$ than for those with higher luminosity. Also, the effect of external heating is more noticeable at longer wavelengths (where apertures/beams are also larger) than at shorter ones; given our chosen apertures, the largest effect occurs at 160 and $870 \mu \mathrm{m}$. We also note that the flux increases due to heating by the ISRF are smallest for the lowest $\rho_{1000}$ value probed, $2.4 \times 10^{-20} \mathrm{~g} \mathrm{~cm}^{-3}$; at $160 \mu \mathrm{m}$, the flux increase is largest for intermediate envelope densities. Finally, the flux increases in the far-IR and submillimeter are far larger for a solar-neighborhood ISRF scaled by factor of 10 than for an unscaled ISRF; for the $L_{\text {tot }}=0.1 L_{\odot}$ models, an unscaled ISRF increases the fluxes from a few percent (at $\lesssim 100 \mu \mathrm{m}$ ) to $50 \%$ (at $870 \mu \mathrm{m}$ ), while an ISRF scaled by a factor of 10 increases these fluxes by $30 \%-75 \%$. Thus, for low-luminosity protostars, up to $\sim 75 \%$ of a protostar's $870 \mu \mathrm{m}$ flux could be due to external heating, if the environment is dominated by an extremely strong ISRF.

To estimate how the contribution of external heating would modify derived model parameters, in Figures 17 and 18 we compare model SEDs that include external heating by an ISRF 10 times stronger than in the solar neighborhood and model SEDs without this additional heating. For the latter, we used models from our model grid and tried to reproduce the SEDs with external heating. For the models with $L_{\text {tot }}=0.1 L_{\odot}$, the effect of external heating can be reproduced by increasing the luminosity by factors of a few, increasing $\rho_{1000}$ by up to an order of magnitude, and increasing the cavity opening angle and inclination angle by a small amount. For the $L_{\text {tot }}=1.0 L_{\odot}$ models, just increasing the reference density by a factor of 2.5 results in a good match to the long-wavelength emission of our externally heated models; however, the shorter-wavelength flux is either under- or overestimated. A better match is achieved with models having the same reference density as the externally heated models, but with slightly larger cavity opening angles and inclination angles, and luminosities about a factor of 2 larger. Thus, if the far-IR and submillimeter fluxes were 

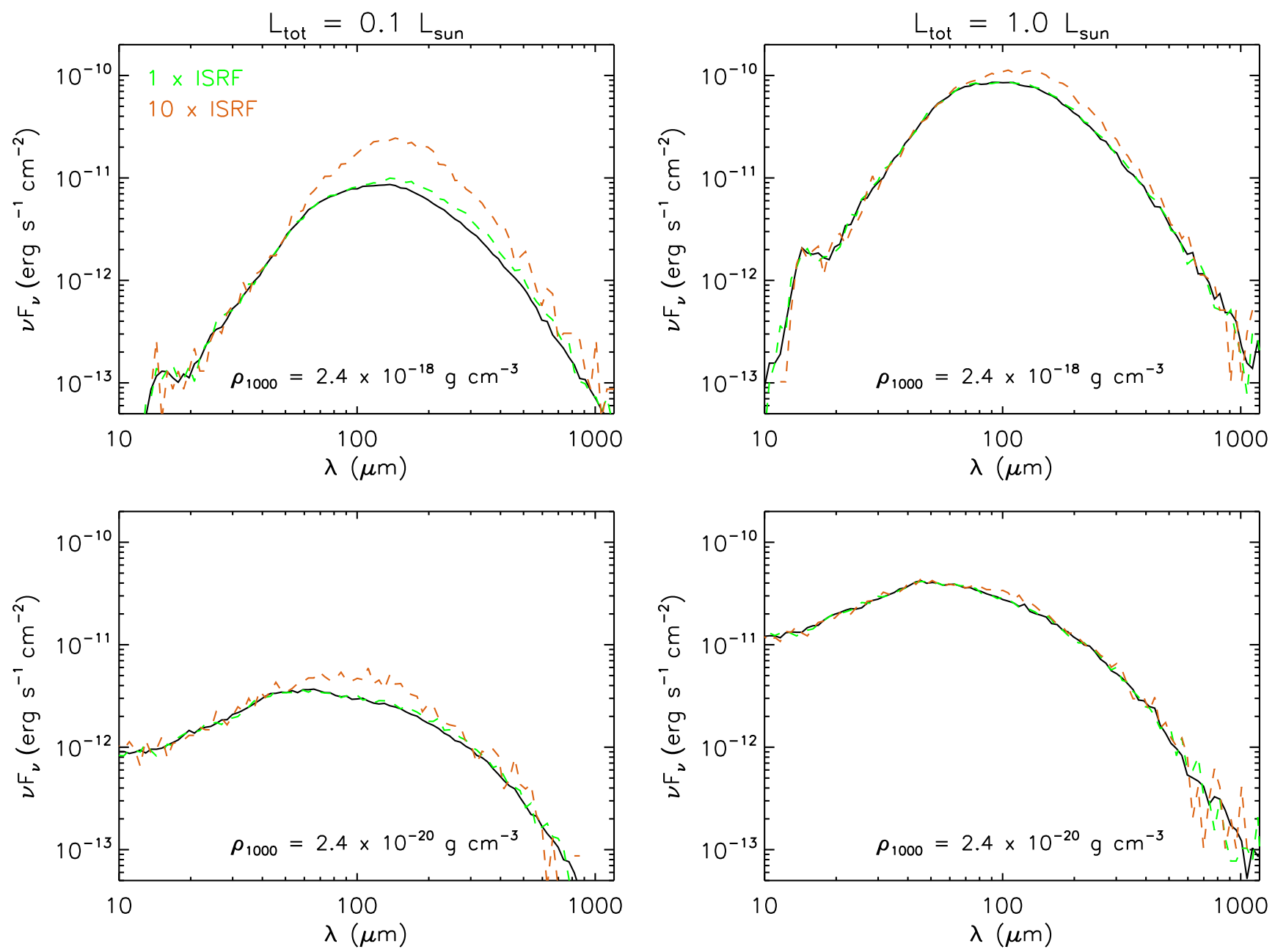

Figure 15. Left: comparison of models with $L_{\mathrm{tot}}=0.1 L_{\odot}, R_{c}=100 \mathrm{au}, \theta=15^{\circ}, \rho_{1000}=2.4 \times 10^{-18} \mathrm{~g} \mathrm{~cm}^{-3}$ (top) or $2.4 \times 10^{-20} \mathrm{~g} \mathrm{~cm}^{-3}$ (bottom), $i=63^{\circ}$, without external heating (black), with external heating by an ISRF equal to that in the solar neighborhood (green dashed line), and with heating by an ISRF 10 times stronger (orange dashed line). Right: similar to the models in the left panels, but these models have $L_{\text {tot }}=1.0 L_{\odot}$.

contaminated by emission resulting from extremely strong external heating, a model fit using models from our grid (which does not include external heating) could overestimate the envelope density by up to an order of magnitude and the luminosity by a factor of $2-5$. The cavity opening and inclination angles would also be more uncertain, but not by much. For a more realistic scenario with more modest external heating (which would also include the effect of local extinction), the effect on model parameters would be smaller.

For the latter point, we explored the effect of extinction on the ISRF by calculating a few more models with $L_{\text {tot }}=0.1 L_{\odot}$, $R_{c}=100 \mathrm{au}, \theta=15^{\circ}, \rho_{1000}=2.4 \times 10^{-18} \mathrm{~g} \mathrm{~cm}^{-3}$, an ISRF 10 times stronger than that in the solar neighborhood, and $A_{V}$ values for the ISRF of 2.5, 10, 20, and 50. The model SEDs are shown in Figure 19. Compared to ISRF heating without any foreground extinction, already $A_{V}=2.5$ causes a decrease by a factor of 1.5-2 in the overall emission at far-IR wavelengths. With $A_{V}$ of 10 and 20, the far-IR emission decreases by factors of up to $\sim 3.5$ and 4 , respectively, compared to a strong ISRF that is not extinguished. The fraction of excess emission due to external heating at $160 \mu \mathrm{m}$ decreases from an average of 0.8 for $A_{V}=0$ (see Figure 16) to 0.6, 0.3, and 0.2 for $A_{V}=2.5,10$, and 20 , respectively. Therefore, considering that typical $A_{V}$ values in Orion are $\sim 10-20 \mathrm{mag}$ (Stutz \& Kainulainen 2015), it is likely that the effect of external heating on model parameters of low-luminosity sources does not exceed a factor of $\sim 2$ in luminosity and $\sim 5$ in envelope density.

\section{FITTING METHOD}

A customized fitting routine determines the best-fit model from the grid for each object in our sample of 330 YSOs (see Sections 2 and 3) using both photometry and, where available, IRS spectroscopy. Ideally, an object has 2MASS, IRAC, IRS, MIPS, PACS, and SABOCA and LABOCA data; in many cases, no submillimeter data are available, and in a few cases the object is too faint to be detected by 2MASS. Of the 330 modeled objects, 40 do not have IRS spectra. As a minimum, objects have some Spitzer photometry and a measured flux value in the PACS $70 \mu \mathrm{m}$ band. No additional data from the literature were included in the fits to keep them homogeneous.

In order to reduce the number of data points contained in the IRS spectral wavelength range (such that the spectrum does not dominate over the photometry) and to exclude ice absorption features in the $5-8 \mu \mathrm{m}$ region and at $15.2 \mu \mathrm{m}$ that are usually observed, but not included in the model opacities, we rebin each IRS spectrum to fluxes at 16 wavelengths. These data points trace the continuum emission and the 10 and $20 \mu \mathrm{m}$ silicate features. Also, when rebinning the spectrum, we smooth over its noisy regions, and we scale the whole spectrum to match the MIPS 

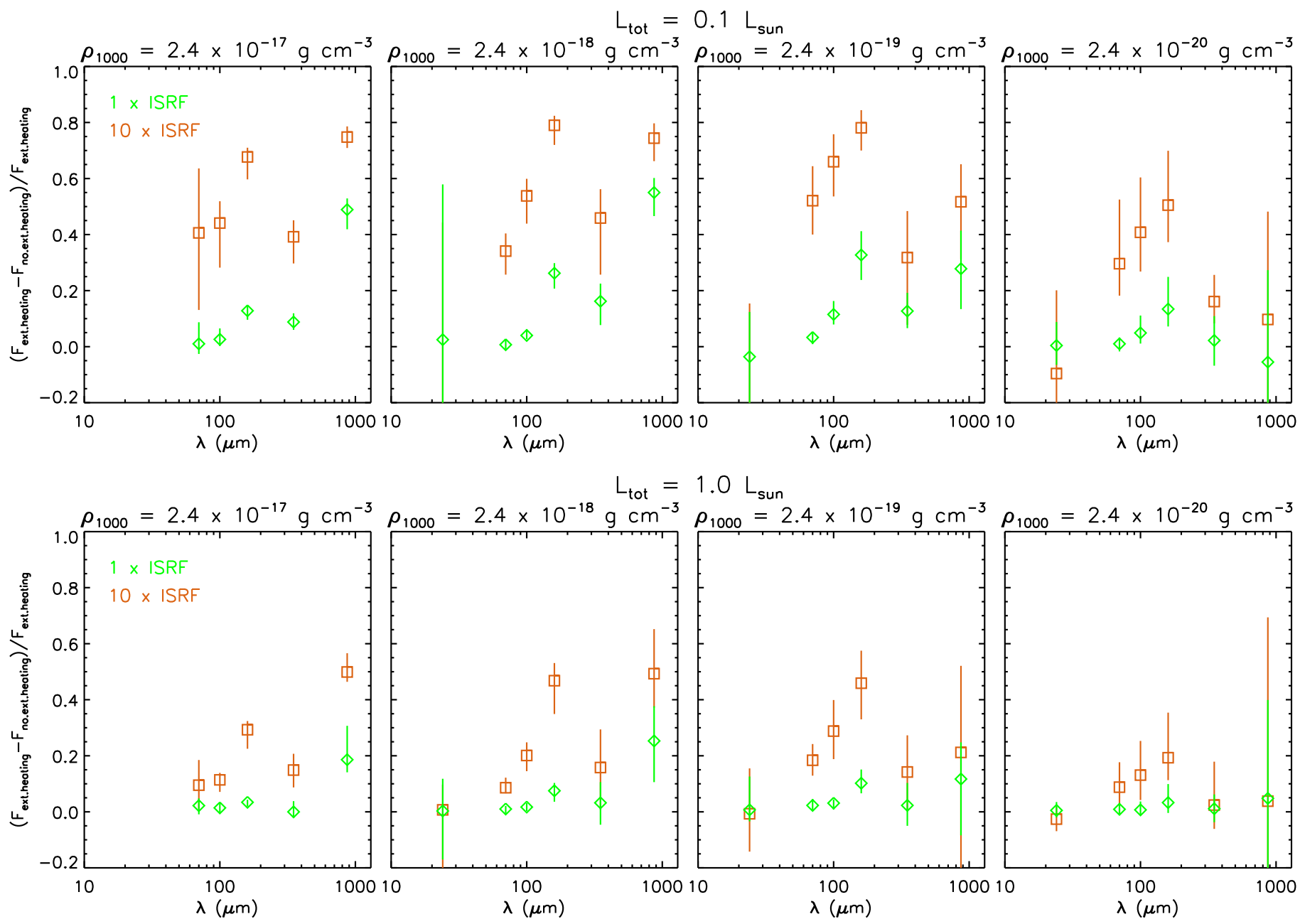

Figure 16. Ratio of the excess emission due to external heating and the emission of the protostar with external heating in different bands, for heating by an ISRF equal to that in the solar neighborhood (green diamonds) and by an ISRF 10 times stronger (orange squares). The vertical lines show the range of flux excess ratios resulting from different viewing angles (inclination angles range from $18^{\circ}$ to $87^{\circ}$ ), while the symbols represent mean values. The top (bottom) panels are for models with $L_{\mathrm{tot}}=0.1(1.0) L_{\odot}$. The four columns correspond to the four reference densities probed.
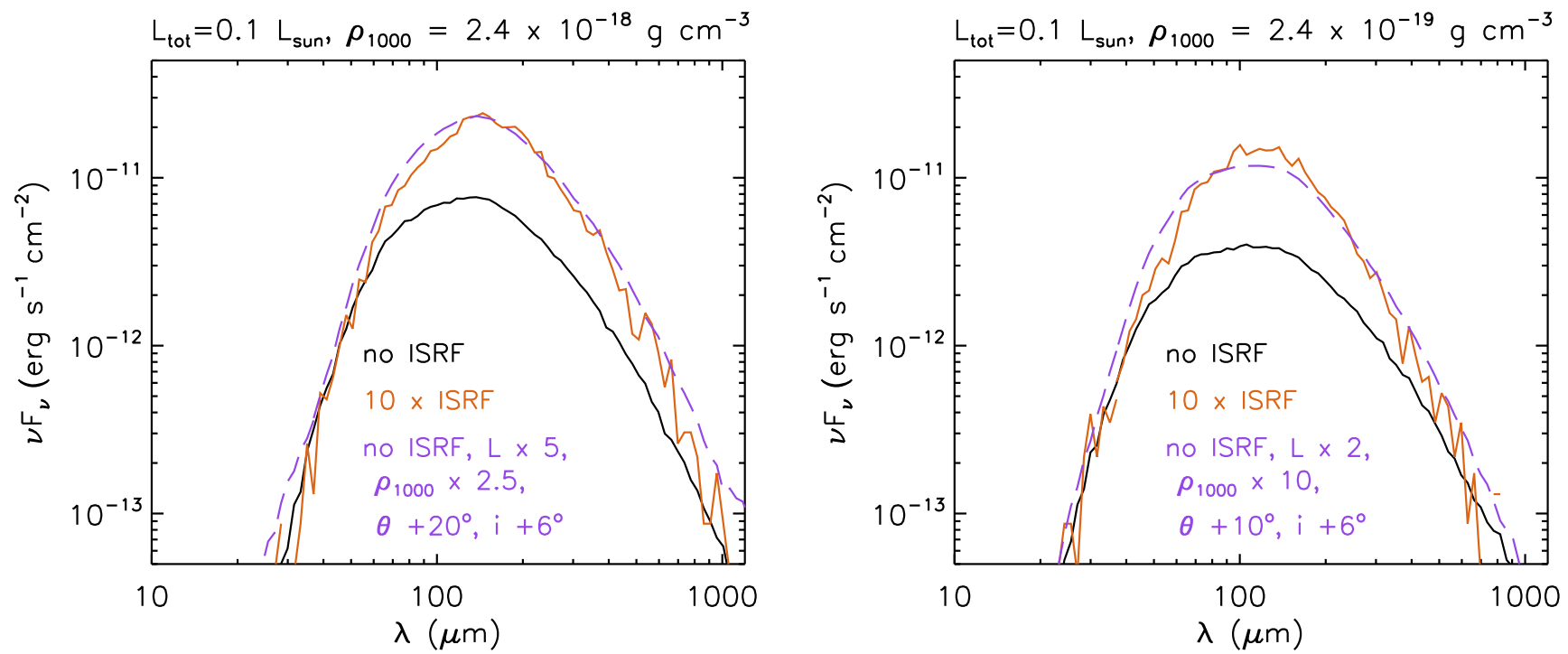

Figure 17. Black and orange lines: SEDs for models with $L_{\mathrm{tot}}=0.1 L_{\odot}, R_{c}=100 \mathrm{au}, \theta=15^{\circ}, i=75^{\circ}$, reference densities $\rho_{1000}=2.4 \times 10^{-18} \mathrm{~g} \mathrm{~cm}{ }^{-3}($ left) and $2.4 \times 10^{-19} \mathrm{~g} \mathrm{~cm}^{-3}$ (right), without external heating (black) and with heating by an ISRF scaled by a factor of 10 (orange). The purple dashed lines show SEDs from our model grid (which does not include external heating) with model parameters changed as indicated in the figure label; these models were chosen to closely match the model SEDs with external heating. 

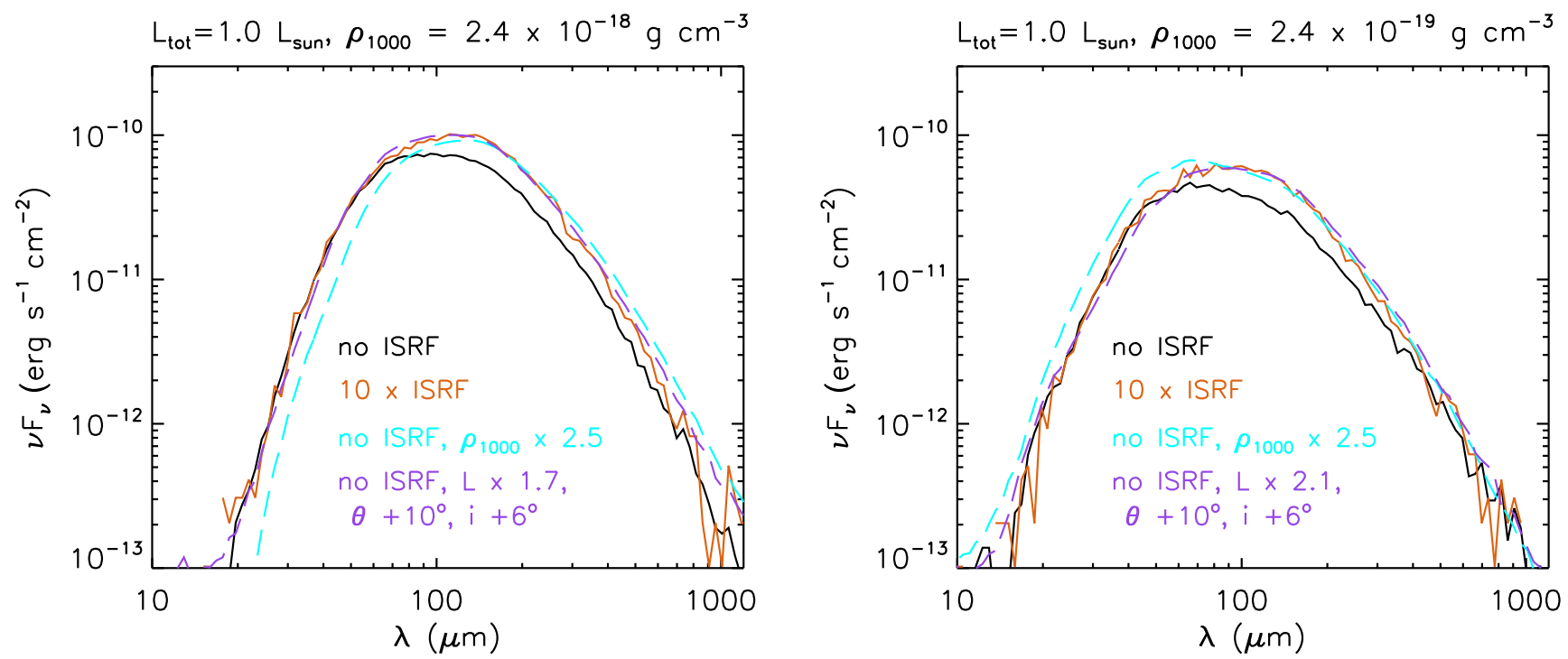

Figure 18. Similar to Figure 17, but for model SEDs with $L_{\text {tot }}=1.0 L_{\odot}$ (black and orange lines). The light blue and purple dashed lines show SEDs from our model grid (no external heating) with the same model parameters as shown except for a reference density 2.5 times higher (light blue) and $\theta=25^{\circ}, i=81^{\circ}$, and a higher luminosity (purple).

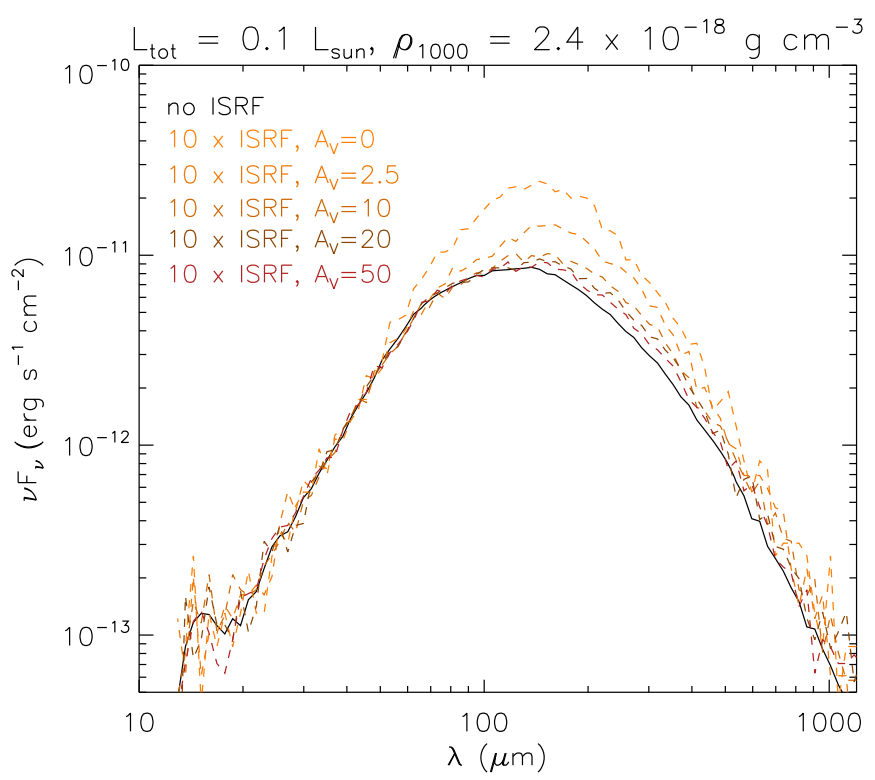

Figure 19. Models with $L_{\mathrm{tot}}=0.1 L_{\odot}, R_{c}=100 \mathrm{au}, \theta=15^{\circ}$, $\rho_{1000}=2.4 \times 10^{-18} \mathrm{~g} \mathrm{~cm}^{-3}, i=63^{\circ}$, without external heating (black), with external heating by an ISRF 10 times stronger than in the solar neighborhood (orange to brown, dashed lines), and different amounts of extinction applied to the ISRF (from $A_{V}=2.5$ to $A_{V}=50$, top to bottom).

$24 \mu \mathrm{m}$ flux if a similar deviation is also seen at the IRAC 5.8 and $8 \mu \mathrm{m}$ bands and is larger than $10 \%$. Figure 20 shows three examples of our IRS spectra with the rebinned fluxes overplotted. Our selection of 16 IRS data points in addition to at most 13 photometric points spread from 1.1 to $870 \mu \mathrm{m}$ puts more emphasis on the mid-IR spectral region in the fits. This wavelength region is better sampled by observations; most of the emission is thermal radiation from the protostellar envelope and disk (as opposed to some possible inclusion of scattered light or thermal emission from surrounding material at shorter and longer wavelengths, respectively) and contains the $10 \mu \mathrm{m}$ silicate feature, which crucially constrains the SED fits. As a result, most models are expected to reproduce the mid-IR fluxes well and might fit more poorly in the near-IR and submillimeter.
To directly compare observed and model fluxes, we create model SEDs with data points that correspond to those obtained from observations, from both photometry and IRS spectroscopy. For the former, the model fluxes are not only derived from the same apertures as the data (see section 4.2), but also integrated over the various filter bandpasses, thus yielding model photometry. For the latter, the model fluxes are interpolated at the same 16 wavelength values as the IRS spectra.

Since the model grid contains a limited number of values for the total luminosity (eight), but the objects we intend to fit have luminosities that likely do not correspond precisely to these values, we include scaling factors for the luminosity when determining the best-fit model. As long as these scaling factors are not far from unity, they are expected to yield SEDs that are very similar to those obtained from models using the scaled luminosity value as one of the input parameters. The scaling factor can also be related to the distance of the source; for all model fluxes, a distance of $420 \mathrm{pc}$ is assumed, but in reality the protostars in our sample span a certain (presumably small) range of distances along the line of sight. For example, a 10\% change in distance would result in a $\sim 20 \%$ change in flux values (scaling factors of 0.83 or 1.23). Here we report luminosities assuming a distance of $420 \mathrm{pc}$.

In addition to scaling factors, each model SED can be extinguished to account for interstellar extinction along the line of sight. We use two foreground extinction laws from McClure (2009) that were derived for star-forming regions: one applies to $0.76 \leqslant A_{J}<2.53$ (or $0.3 \leqslant A_{K}<1$ ), and the other one to $A_{J} \geqslant 2.53$ (or $A_{K} \geqslant 1$ ). For $A_{J}<0.76$, we use a spline fit to the Mathis $R_{V}=5$ curve (Mathis 1990). Since the three laws apply to different extinction environments, we use a linear combination of them to achieve a smooth change in the extinction law from the diffuse interstellar medium to the dense regions within molecular clouds. Thus, to find a best-fit model for a certain observed SED, the model fluxes $F_{\text {mod }}(\lambda)$ are scaled and extinguished as follows:

$$
F_{\text {obs }}(\lambda)=s F_{\text {mod }}(\lambda) 10^{-0.4 A_{\lambda}},
$$

where $F_{\text {obs }}(\lambda)$ and $F_{\text {mod }}(\lambda)$ are the observed and model fluxes, respectively, $s$ is the luminosity scaling factor, and $A_{\lambda}$ is the extinction at wavelength $\lambda$. We use three reddening laws, $k_{\lambda}=A_{\lambda} / A_{J}$; by denoting them with the subscripts 1,2 , and 3 , 

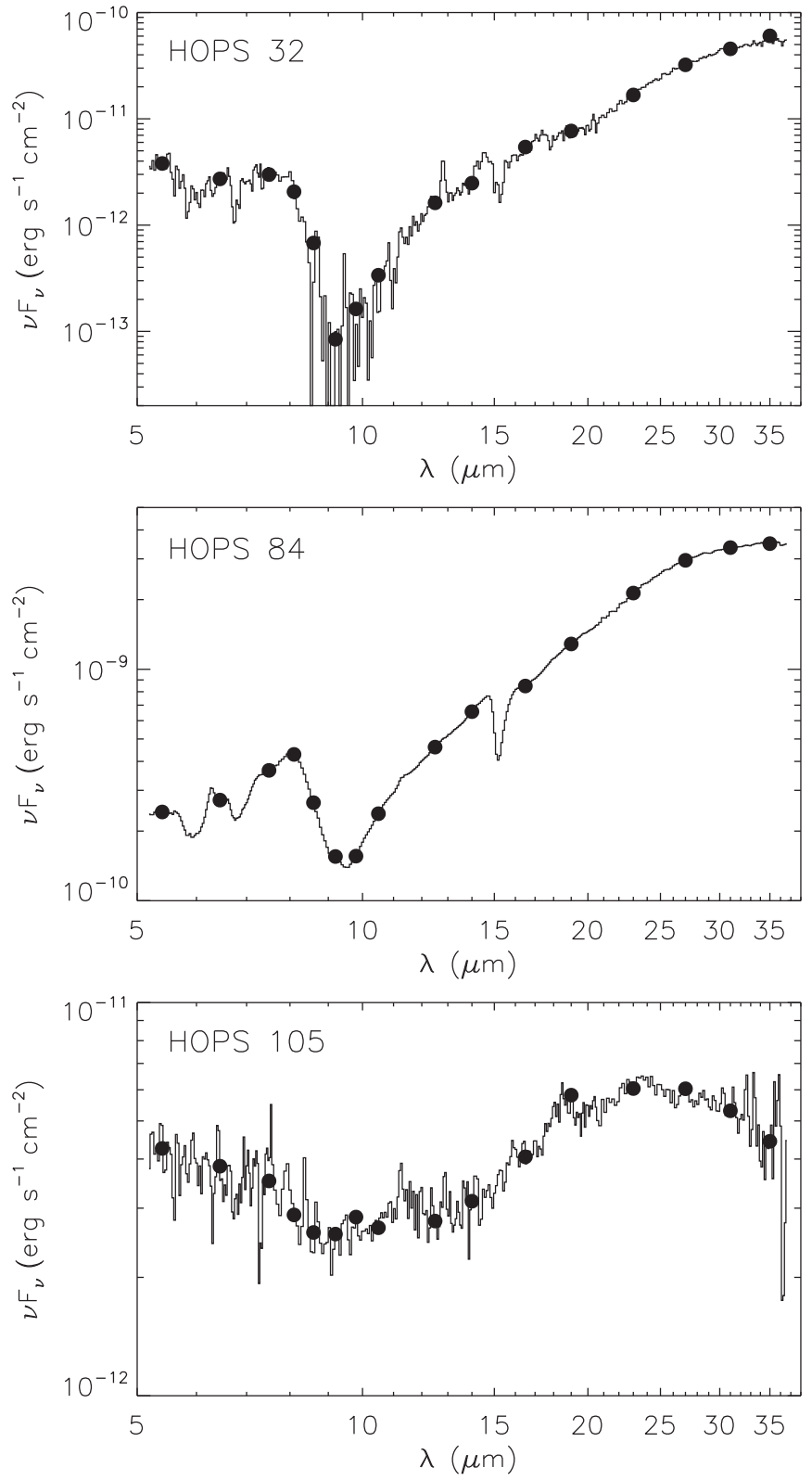

Figure 20. Three IRS spectra, one for HOPS 32 (Class 0 protostar; top), one for HOPS 84 (Class I protostar; middle), and one for HOPS 105 (flat-spectrum source; bottom), overlaid with the rebinned data points (filled circles) used by the fitting routine. Note the different flux ranges on the $y$-axis in the three panels and thus the big differences in slopes among the three spectra.

$A_{\lambda}$ in the above equation becomes

$$
\begin{gathered}
A_{\lambda}=A_{J} k_{1, \lambda} \quad \text { for } A_{J}<0.76 \\
A_{\lambda}=0.76 k_{1, \lambda}+\left(A_{J}-0.76\right) k_{2, \lambda} \\
\quad \text { for } 0.76<A_{J}<2.53 \\
A_{\lambda}=0.76 k_{1, \lambda}+2.53 k_{2, \lambda}+\left(A_{J}-2.53\right) k_{3, \lambda} \\
\quad \text { for } A_{J}>2.53 .
\end{gathered}
$$

Thus, Equation (3) can be written as

$$
\begin{aligned}
& 2.5 \log \left(F_{\text {mod }}(\lambda) / F_{\text {obs }}(\lambda)\right)=A_{J} k_{1, \lambda}-2.5 \log (s) \\
& \quad \text { for } A_{J}<0.76 \\
& 2.5 \log \left(F_{\text {mod }}(\lambda) / F_{\text {obs }}(\lambda)\right)-0.76\left(k_{1, \lambda}-k_{2, \lambda}\right) \\
& \quad=A_{J} k_{2, \lambda}-2.5 \log (s) \text { for } 0.76<A_{J}<2.53 \\
& 2.5 \log \left(F_{\text {mod }}(\lambda) / F_{\text {obs }}(\lambda)\right)-0.76 k_{1, \lambda}-2.53\left(k_{2, \lambda}-k_{3, \lambda}\right) \\
& =A_{J} k_{3, \lambda}-2.5 \log (s) \text { for } A_{J}>2.53 .
\end{aligned}
$$

These are linear equations in $A_{J}$, with the left-hand side of the equations as the dependent variables and $k_{\lambda}$ as the independent variable. For each regime of $A_{J}$ values, a best-fit line can be determined that yields $A_{J}$ and $-2.5 \log (s)$ from the slope and intercept, respectively, for each model that is compared to the observations.

For each set of model fluxes and observed fluxes, we calculate three linear fits (using linear combinations of the three different extinction laws, as explained above), thus yielding three values for scaling factors and three for the extinction value. If each extinction value is within the bounds of the extinction law that was used and smaller than a certain maximum $A_{J}$ value (which will be discussed below), and the scaling factor is in the range from 0.5 to 2.0 , then the result with the best linear fit will be used. However, if some of the values are not within their boundaries, then combinations of their limiting values are explored, and the set of scaling factor and extinction with the best fit is adopted. For example, if a model has fluxes that are much higher than all observed fluxes, the linear fit described above will likely yield very large extinction values and small scaling factors. In this case the fitter would only accept the smallest possible scaling factor $(0.5)$ and the maximum allowed $A_{J}$ value as a solution (which will still result in a poor fit).

For each object, we allowed the model fluxes to be extinguished up to a maximum $A_{J}$ value derived from column density maps of Orion (Stutz \& Kainulainen 2015; see also Stutz et al. 2010, 2013; Launhardt et al. 2013 for the methodology of deriving $N_{\mathrm{H}}$ from 160 to $500 \mu \mathrm{m}$ maps). We converted the total hydrogen column density from these maps to $A_{V}$ values $\left(A_{V}=3.55 A_{J}\right)$ by using a conversion factor of $1.0 \times 10^{21} \mathrm{~cm}^{-2} \mathrm{mag}^{-1}$ (Winston et al. 2010; Pillitteri et al. 2013). For objects for which no column density could be derived, we set the maximum $A_{J}$ value to 8.45 (which corresponds to $A_{V}=30$ ).

After returning a best-fit scaling factor and extinction value for each model, each data point is assigned a weight, and the goodness of the fit is estimated with

$$
R=\frac{\sum_{i=1}^{N} w_{i}\left|\ln \left(\frac{F_{\mathrm{obs}}\left(\lambda_{i}\right)}{F_{\mathrm{mod}}\left(\lambda_{i}\right)}\right)\right|}{N}
$$

where $w_{i}$ are the weights, $F_{\text {obs }}\left(\lambda_{i}\right)$ and $F_{\bmod }\left(\lambda_{i}\right)$ are the observed and the scaled and extinguished model fluxes, respectively, and $N$ is the number of data points (see Fischer et al. 2012). Thus, $R$ is a measure of the average, weighted, logarithmic deviation between the observed and model SED. It was introduced by Fischer et al. (2012) since the uncertainty of the fit is dominated by the availability of models in the grid (i.e., the spacing of the models in SED space) and not by the measurement uncertainty of the data, making the standard $\chi^{2}$ analysis less useful. Also, a statistic that measures deviations between models and data in log space more closely resembles the assessment done by eye when comparing models and observed SEDs in $\log \left(\lambda F_{\lambda}\right)$ versus $\lambda$ plots. We set the weights $w_{i}$ to the inverse of the estimated fractional uncertainty of each data point; so, for photometry at wavelengths below $3 \mu \mathrm{m}$ they are equal to $1 / 0.1$, between 3 and $60 \mu \mathrm{m}$ they are $1 / 0.05$, at 70 and $100 \mu \mathrm{m}$ they are $1 / 0.04$, at $160 \mu \mathrm{m}$ the weight is $1 / 0.07$, and for photometry at 350 and $870 \mu \mathrm{m}$ they are $1 / 0.4$ and 
$1 / 0.2$, respectively. For fluxes from IRS spectra the weights are $1 / 0.075$ for wavelength ranges $8-12 \mu \mathrm{m}$ and $18-38 \mu \mathrm{m}$, while they are $1 / 0.1$ for the $5-8 \mu \mathrm{m}$ and $12-18 \mu \mathrm{m}$ regions. These IRS weights are also multiplied by 1.5 for high signal-to-noise spectra and by 0.5 for noisy spectra. In this way those parts of the IRS spectrum that most constrain the SED, the $10 \mu \mathrm{m}$ silicate absorption feature and slope beyond $18 \mu \mathrm{m}$, are given more weight; for high-quality spectra, the weights in these wavelength regions are the same as for the 3-60 $\mu \mathrm{m}$ photometry.

For small values, $R$ measures the average distance between model and data in units of the fractional uncertainty. In general, the smaller the $R$ value, the better the model fit, but protostars with fewer data points can have small $R$ values, while protostars with some noisy data can have larger $R$ values (but still an overall good fit). We find a best-fit model for each object, but we also record all those models that lie within a certain range of $R$ values from the best-fit $R$. These models give us an estimate on how well the various model parameters are constrained (see Section 6.4).

Our model grid is used to characterize the parameters that best describe the observed SED of each object; the $R$ values rank the models for each object and thus can be used to derive best-fit parameters, as well as estimates of parameter ranges. In several instances, better fits could be achieved if the model parameters were further adjusted, for example, by testing more values of cavity opening angle or shape, or even changing the opacities (see, e.g., HOPS 68 [Poteet et al. 2011], HOPS 223 [Fischer et al. 2012], HOPS 59, 60, 66, 108, 368, 369, 370 [Adams et al. 2012], HOPS 136 [Fischer et al. 2014], and HOPS 108 [Furlan et al. 2014]). However, for protostars that are well fit with one of the models from the grid or for which the grid yields a narrow range of parameter values, it is unlikely that a more extended model grid would yield much different best-fit parameters. Overall, our model fits yield good estimates of envelope parameters for a majority of the sample, and thus we can analyze the protostellar properties of our HOPS targets in a statistical manner.

\section{RESULTS OF THE MODEL FITS}

The best-fit parameters resulting from our models can be found in Table 1, and Figure 1 shows the SEDs and best fits for our sample. In this section we give an overview of the quality of the fits, the distributions of the best-fit model parameters, both for the sample as a whole and separated by SED class, the parameter uncertainties, and the various degeneracies between model parameters.

\subsection{Quality of the Fits}

Figure 21 displays the histogram of $R$ values of the best model fits for the 330 objects in our HOPS sample that have Spitzer and Herschel data (more than two data points at different wavelengths) and are not contaminants (see Section 2). The median $R$ value is 3.10 , while the mean value is 3.29 . Fitting a Gaussian to the histogram at $R \leqslant 7$ yields 3.00 and 2.24 as the center and FWHM of the Gaussian, respectively. The distribution of $R$ values implies that, on average, the model deviates by about three times the average fractional uncertainty from the data. This is not unexpected, given that we fit models from a grid to observed SEDs that span almost three orders of magnitude in wavelength range, with up to 29 data points. The fewer the data points, the easier it is to achieve a good fit; in fact, the eight protostars with $R<1$, HOPS 371, 391, 398, $401,402,404,406$, and 409, have SEDs with measured flux values at only 4-5 points. Starting at $R$ values of about $1, R$ can be used as an indicator of the goodness of fit. However, in some cases a noisy IRS spectrum can increase the $R$ value of a fit that, judged by the photometry alone, does not deviate much from the observed data points. In other cases, mismatches between different data sets, like offsets between the IRAC fluxes and the IRS spectrum, can result in larger $R$ values.

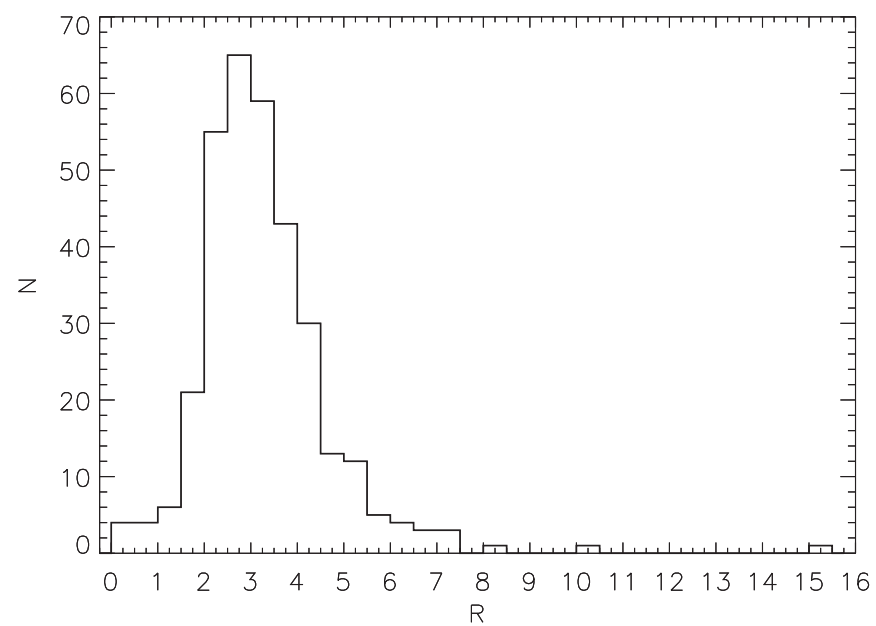

Figure 21. Histogram of the $R$ values of the best fits of the 330 YSOs in the HOPS sample that have Spitzer and Herschel detections.

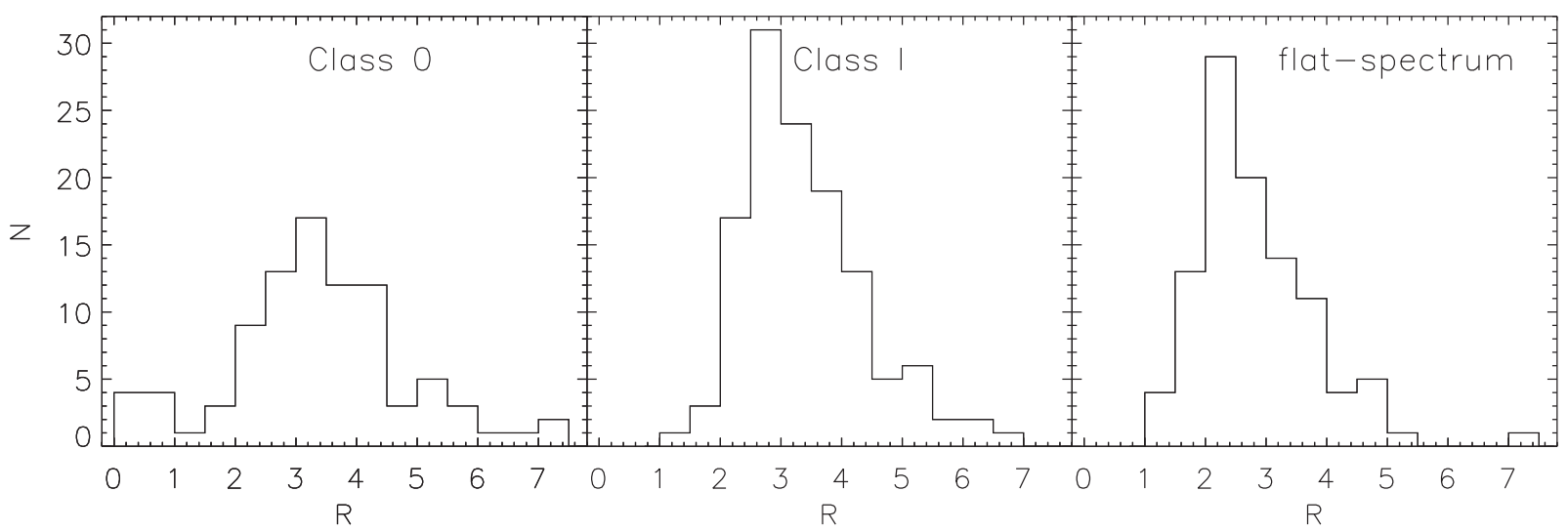

Figure 22. Histograms of the $R$ values of the best fits shown separately for the three classes of objects (Class 0 , I, and flat-spectrum). The three fits with $R>8$ (two Class 0 protostars, one Class I protostar) are not shown. 
These might be interesting protostars affected by variability and are thus ideal candidates for follow-up observations.

When looking at the SED fits in Figure 1 (and the corresponding $R$ values in Table 1), we estimate that an $R$ value of up to $\sim 4$ can identify a reliable fit (with some possible discrepancies between data and model in certain wavelength regions). When $R$ gets larger than about 5, the discrepancy between the fit and the observed data points usually becomes noticeable; the fit might still reproduce the overall SED shape but deviate substantially from most measured flux values.

In Figure 22, we show the histogram of $R$ values separately for the three main protostellar classes in our sample. The median $R$ value decreases from 3.27 for the Class 0 protostars to 3.18 for the Class I protostars to 2.58 for the flat-spectrum sources. There are 4 Class 0 protostars and 4 Class I protostars with $R$ values between 1.0 and 2.0, but 17 flat-spectrum sources in this $R$ range. These numbers translate to $17 \%$ of the flatspectrum sources in our sample, $4 \%$ of the Class 0 protostars, and $3 \%$ of the Class I protostars. When examining objects' $R$ values between 2.0 and 4.0, there are 51 Class 0 protostars (55\% of Class 0 protostars in the sample), 91 Class I protostars ( $73 \%$ of the Class I sample), and 74 flat-spectrum sources (73\% of the flat-spectrum sample).

Thus, close to $90 \%$ of flat-spectrum sources are fit reasonably well $(R$ values $<4)$, representing the largest fraction among the different classes of objects in our sample. This could be a result of their source properties being well represented in our model grid, but also lack of substantial wavelengthdependent variability (see, e.g., Günther et al. 2014), which, if present, would make their SEDs more difficult to fit. About three-quarters of Class I protostars also have best-fit models with $R<4$; this fraction drops to about two-thirds for the Class 0 protostars. The latter group of objects often suffers from more uncertain SEDs owing to weak emission at shorter wavelengths (which, e.g., results in a noisy IRS spectrum); they might also be more embedded in extended emission, such as filaments, which can contaminate the far-IR to submillimeter fluxes. Another factor that could contribute to poor fits is their presumably high envelope density, which places them closer to the limit in parameter space probed by the model grid. Overall, $75 \%$ of the best-fit models of the protostars in our sample have $R<4$.

When examining the SED fits of objects with $R$ values larger than 5.0, several have very noisy IRS spectra (HOPS 19, 38, $40,95,164,278,316,322,335,359)$. In a few cases the measured PACS 100 and $160 \mu \mathrm{m}$ fluxes seem too high compared to the best-fit model (e.g., HOPS 189), which could be an indication of contamination by extended emission surrounding the protostar.

Of particular interest are objects where variability likely plays a role in a poor fit. As mentioned in Section 3, variability among protostars is common; we found in Appendix A that about $5 \%$ of our targets display noticeable ( $\gtrsim 50 \%)$ mismatches between the IRS, IRAC, and MIPS fluxes that could be due to intrinsic variability. The SED fits of objects for which the flux mismatches between IRS and IRAC and between IRS and MIPS are different are particularly affected, since in that case we did not scale the IRS spectrum to match the MIPS $24 \mu \mathrm{m}$ flux. HOPS 228 exemplifies such a case: there is a clear discrepancy between the IRAC and IRS fluxes (a factor of 2.1-2.7) and also between MIPS $24 \mu \mathrm{m}$ and IRS (a factor of 0.8 ; even though the fit gives more weight to the IRS data,

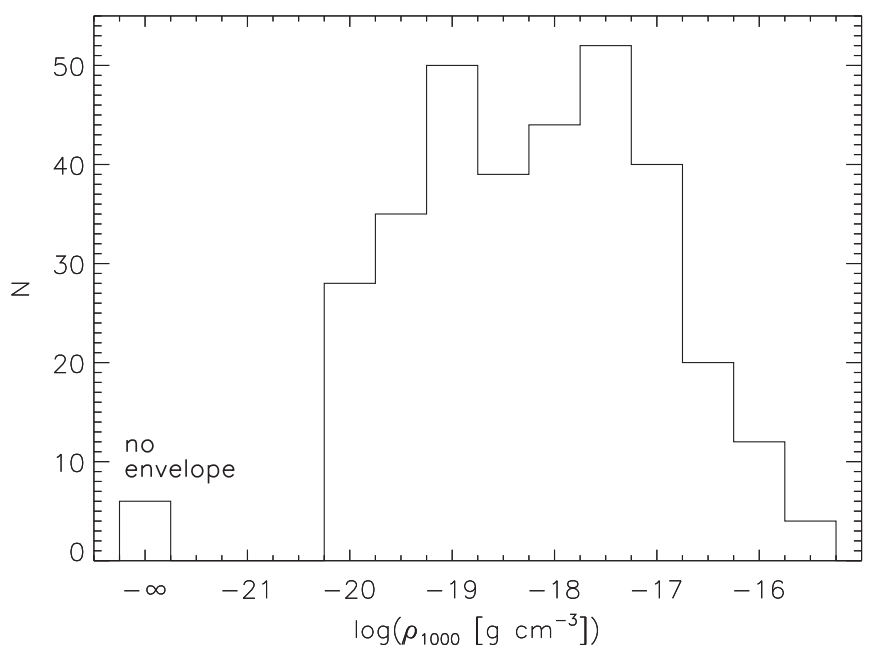

Figure 23. Histogram of the envelope reference density $\rho_{1000}$ of the best fits for the 330 targets in our sample.

they are not fit well, especially the silicate absorption feature. The $R$ value of 5.74 for the fit of HOPS 228 reflects the discrepant data sets and poor fit. HOPS 223 is another case where the IRS fluxes do not match the shorter-wavelength data (they are more than an order of magnitude larger); however, it is a known FU Ori source (see Fischer et al. 2012), and the SED presented here contains both pre- and post-outburst data. The model fit is very poor, which can also be gauged by the $R$ value of 8.41 .

There are also objects with overall good fits whose SEDs show discrepancies that may be signs of variability or contamination. For example, for the Class I protostar HOPS 71 the IRAC fluxes are a factor of 1.8-2.4 lower than the IRS fluxes in the 5-8 $\mu \mathrm{m}$ region, and also the MIPS flux is about $20 \%$ lower. The best-fit model $(R=3.63)$ fits the SED extremely well beyond about $6 \mu \mathrm{m}$, with some discrepancy at shorter wavelengths. There is a source just $11^{\prime \prime}$ from HOPS 71 that is detected in 2MASS and Spitzer data, but not by PACS; this object, HOPS 72, is likely an extragalactic object (see Appendix C.2.2) that could contaminate the IRS fluxes. Thus, in this case, wavelength-dependent contamination by a companion could explain the discrepancies observed in the SED.

Another example is HOPS 124, which is a deeply embedded Class 0 protostar. For this object, the mismatch between IRS and IRAC and MIPS fluxes decreases with increasing wavelength (from a factor of 2.5 to a factor of 1.4); for the SED fit, the IRS spectrum was scaled by 0.7 to match the MIPS $24 \mu \mathrm{m}$ flux. As with HOPS 71, there is a nearby source that could contaminate some of the fluxes, especially at shorter wavelengths: HOPS 125, a flat-spectrum source, lies 9". 8 from HOPS 124 and is brighter than HOPS 124 out to $\sim 20 \mu \mathrm{m}$, but then much fainter at longer wavelengths. The best-fit model of HOPS $124(R=2.43)$ matches the mid- to far-IR photometry and also most of the IRS spectrum well.

As an example of a probably variable flat-spectrum source, HOPS 132 has IRAC fluxes that lie a factor of 1.3-1.7 above those of IRS and a MIPS $24 \mu \mathrm{m}$ flux that is a factor of 0.6 lower. It does not have a close companion; the nearest HOPS source, HOPS 133, is 27" away. The IRS spectrum was not scaled, and since the SED fitter gave more weight to the spectrum, it is fit well, but the IRAC photometry is 
underestimated and the MIPS photometry overestimated. Nonetheless, the $R$ value of the best fit is 2.87 .

Overall, the SED fits of objects that are likely variable or suffer from some contamination are less reliable, but it is not always clear from the $R$ value of the best fit. The SED fitting procedures assume that the protostars are not variable, so when large mismatches between different data sets are present, the fit will appear discrepant with at least some of the observed data points, but the $R$ value would not end up particularly high if, e.g., the IRS spectrum was fit exceptionally well. However, given the data sets we have for these protostars, our SED fits will still yield the best possible estimate for the protostellar parameters describing these systems.

\subsection{Overview of Derived Parameters}

The histogram of best-fit $\rho_{1000}$ values (which is the density of the envelope at $1000 \mathrm{au}$; see Section 4.1) is shown in Figure 23. The median value of the distribution amounts to $5.9 \times 10^{-19} \mathrm{~g} \mathrm{~cm}^{-3}$; this corresponds to a $\rho_{1}$ value of $1.9 \times 10^{-14} \mathrm{~g} \mathrm{~cm}^{-3}$. There is a spread in values: 69 objects have densities $\rho_{1000}$ smaller than $5.0 \times 10^{-20} \mathrm{~g} \mathrm{~cm}^{-3}$ (6 of them have actually no envelope), 89 fall in the $5.0 \times 10^{-20}$ to $5.0 \times 10^{-19} \mathrm{~g} \mathrm{~cm}^{-3}$ range, 96 are between $5.0 \times 10^{-19}$ and $5.0 \times 10^{-18} \mathrm{~g} \mathrm{~cm}^{-3}, \quad 60$ between $5.0 \times 10^{-18}$ and $5.0 \times 10^{-17} \mathrm{~g} \mathrm{~cm}^{-3}$, and 16 have $\rho_{1000}$ values larger than $5.0 \times 10^{-17} \mathrm{~g} \mathrm{~cm}^{-3}$.

We also calculated the envelope mass $\left(M_{\text {env }}\right)$ within 2500 au for the best-fit models (see Figure 24 for their distribution). The 2500 au radius is close to half the FWHM of the PACS $160 \mu \mathrm{m}$ beam at the distance of Orion (i.e., $\left.\sim 6^{\prime \prime}\right)$ and thus roughly represents the spatial extent over which we measure the SEDs. This envelope mass is determined from the integrated envelope density of our best-fit models, with allowances made for outflow cavities, and thus only valid in the context of our models. The median envelope mass within 2500 au amounts to $0.029 M_{\odot}$. The majority of protostars have model-derived masses in the inner 2500 au of their envelopes around $0.1 M_{\odot}$; just 22 objects have $M_{\text {env }}(<2500 \mathrm{au})$ larger than $1.0 M_{\odot}$. Of the 330 modeled objects, 291 have $M_{\text {env }}$ $(<2500 \mathrm{au})$ smaller than $0.5 M_{\odot}$ ( 6 of these 291 objects have no envelope).

Figure 25 contains the histogram of the total luminosities derived from the best-fit models. These luminosities consist of the stellar, disk accretion, and accretion shock components. The median total luminosity amounts to $3.02 L_{\odot}$, while the values cover four orders of magnitude, from $0.06 L_{\odot}$ (for HOPS 336) to $607 L_{\odot}$ (for HOPS 288 and 361). Since the minimum and maximum values for the total luminosity in our grid amount to 0.1 and $303.5 L_{\odot}$, respectively, and our scaling factors range from 0.5 to 2.0 , our fitting procedure can return best-fit luminosities that range from 0.05 to $607 L_{\odot}$. Thus, two protostars are reaching the upper limit allowed for total luminosities in our grid; it is possible that even better fits could be achieved by increasing the luminosity further.

From the distribution of best-fit outer disk radii in Figure 26, it is apparent that most protostars are fit by small disks whose radius is only $5 \mathrm{au}$. Since the outer disk outer radius is the centrifugal radius in our models, infalling material from the envelope tends to accumulate close to the star for most sources. Thus, the disk radius is tied to the envelope structure; a small centrifugal radius implies higher envelope densities at smaller radii and a less flattened envelope structure. The median disk

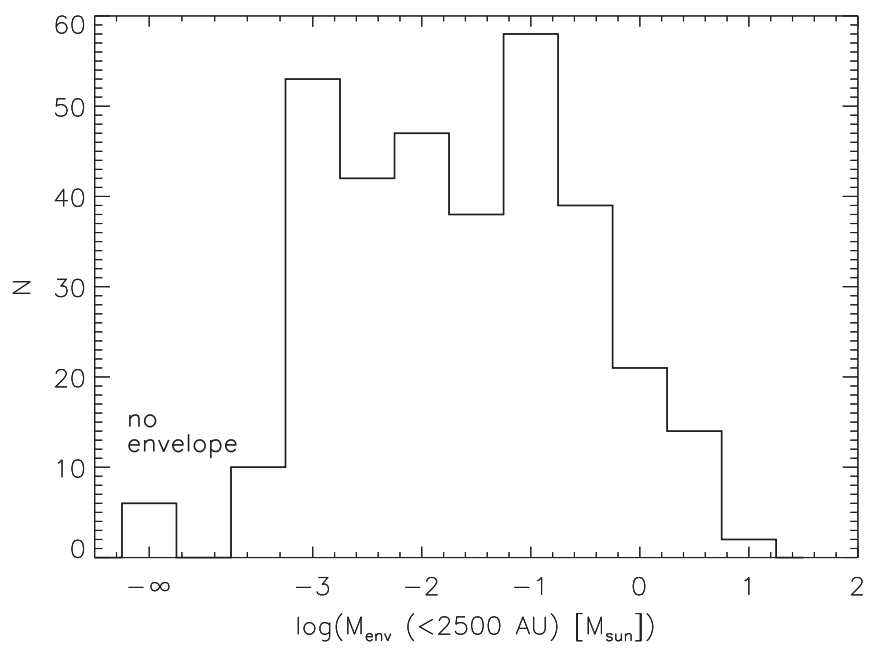

Figure 24. Histogram of the envelope mass within 2500 au derived for the best fits for the 330 targets in our sample.

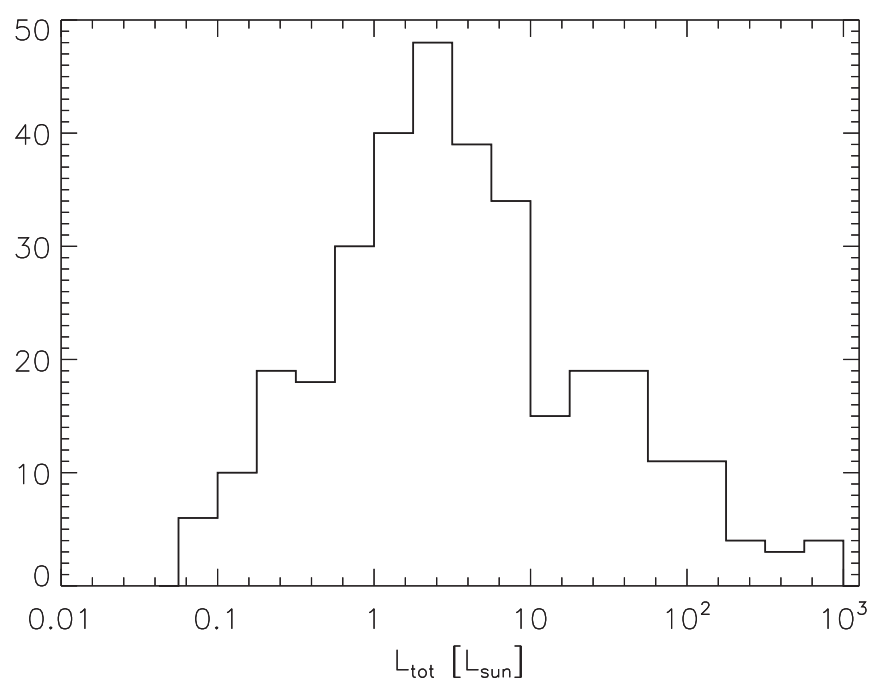

Figure 25. Histogram of the total luminosities of the best fits for the 330 targets in our sample.

radius is $50 \mathrm{au}$, but the number of objects with disk radii $\geqslant 50$ au is roughly evenly split among the values of 50, 100, and $500 \mathrm{au}$.

The distribution of best-fit cavity opening angles is displayed in Figure 27. Most protostars seem to have either very small $\left(5^{\circ}\right)$ or very large $\left(45^{\circ}\right)$ cavities; the median value is $25^{\circ}$. When dividing the envelope densities by cavity opening angle (see Figure 28, left column), differences emerge: the distributions of $\rho_{1000}$ values are significantly different when comparing objects with $\theta=5^{\circ}$ and $\theta \geqslant 35^{\circ}$, objects with $\theta=15^{\circ}$ and $\theta \geqslant 25^{\circ}$, and objects with $\theta=25^{\circ}$ and $\theta=45^{\circ}$. The KolmogorovSmirnov (K-S) tests yield significance levels that these subsamples are drawn from the same parent population of $\lesssim 0.015$. Thus, there seems to be a difference in the distribution of envelope densities among the best-fit models with smaller cavity opening angles and those with larger cavities. Protostars with larger cavities $\left(\geqslant 35^{\circ}\right)$ tend to have higher envelope densities (their median $\rho_{1000}$ values are about an order of magnitude larger compared to objects with cavities $\leqslant 15^{\circ}$ ).

Figure 28 (middle column) also shows the distribution of total luminosities for the different cavity opening angles. The only significant difference can be found for the $\theta=5^{\circ}$ 


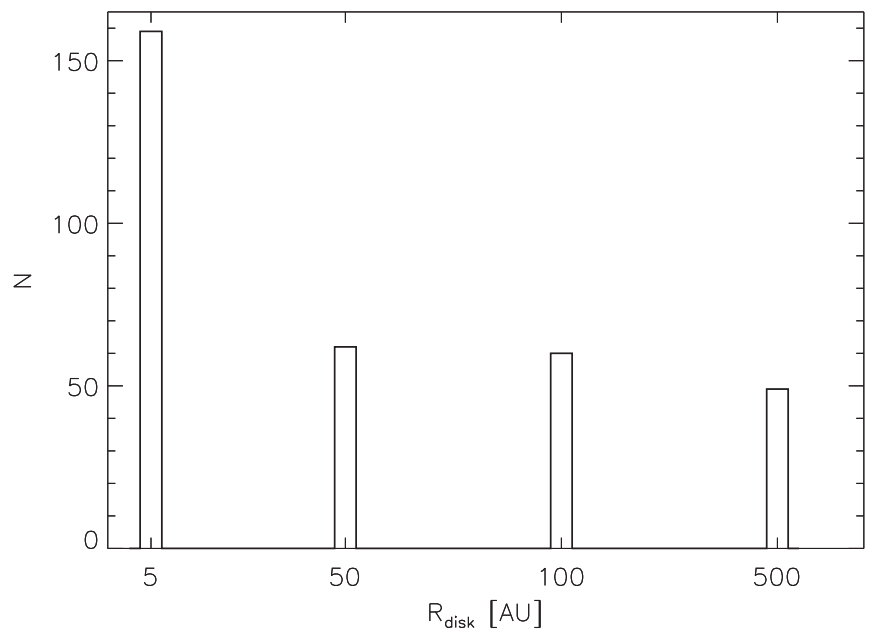

Figure 26. Histogram of the disk radii of the best fits for the 330 targets in our sample.

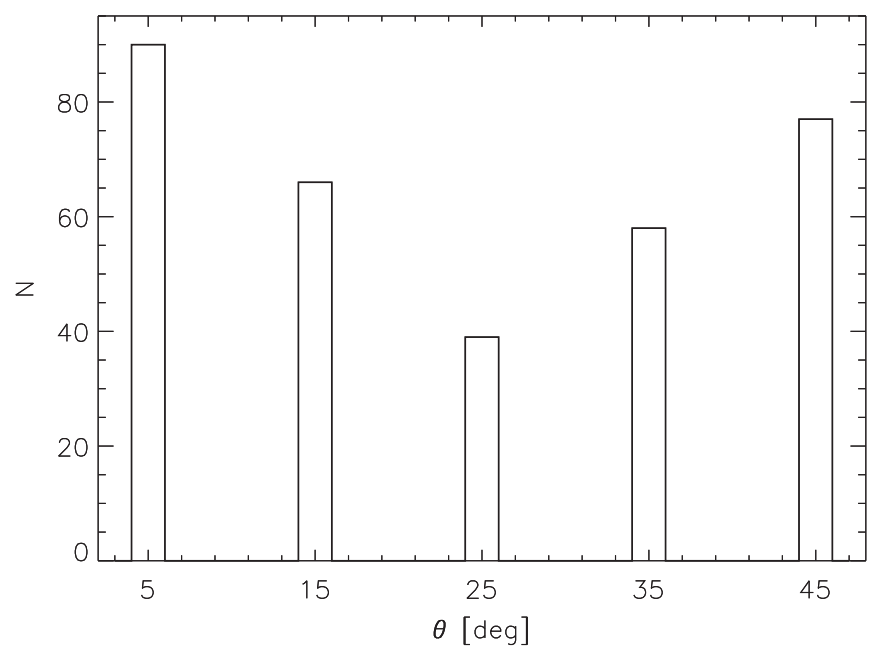

Figure 27. Histogram of the cavity opening angles of the best fits for the 330 targets in our sample.

histogram as compared to the histograms for larger $\theta$ values (K$\mathrm{S}$ test significance level $\lesssim 0.03$ ); the luminosities of models with $\theta=5^{\circ}$ have a different distribution, and also their median value is $1.45 L_{\odot}$, as compared to $\sim 3-5 L_{\odot}$ for the models with larger cavities. So, protostars with small cavities seem to have lower total luminosities.

The distribution of centrifugal radii for different cavity opening angles (right column in Figure 28) shows that, independent of cavity size, most objects have $R_{\text {disk }}=5$ au. However, the distribution among the four different disk radii becomes flatter for the largest cavity opening angles; the histograms for $\theta=35^{\circ}$ and $\theta=45^{\circ}$ are very similar (K-S test significance level of 0.98). There is also no significant difference (K-S test values $>0.075$ ) between the $\theta=15^{\circ}$ and $\theta=25^{\circ}$ histograms and between the $\theta=5^{\circ}$ and $\theta \geqslant 35^{\circ}$ histograms. The distributions of disk radii for the other cavity opening angles are all different from one another (K-S test significance levels $<0.015)$. Overall, Figure 28 shows that protostars best fit by models with large cavity opening angles are also fit by models with higher envelope densities and larger centrifugal radii.
In Figure 29, we show the distribution of the inclination angles for the best-fit models. There is a clear concentration of models in the $60^{\circ}-70^{\circ}$ range; the median inclination angle is $63^{\circ}$. This median value is close to $60^{\circ}$, which is where the probability for isotropically distributed inclination angles reaches $50 \%$ (i.e., the probability of observing an inclination angle less than $60^{\circ}$ is the same as the probability of observing $i>60^{\circ}$ ). However, the details of the distributions differ. The cumulative probability of finding an inclination angle less than a certain value, $i_{c}$, is $1-\cos \left(i_{c}\right)$, assuming a random distribution of inclination angles. For inclination angles $i_{1}$ and $i_{2}$, the probability for $i_{1}<i<i_{2}$ is $\cos \left(i_{1}\right)-\cos \left(i_{2}\right)$. Thus, since the inclination angles in our model grid were chosen to be equally spaced in $\cos (i)$ (there are five values $<60^{\circ}$ and five values $>60^{\circ}$ ), one would expect a flat distribution in Figure 29 if the best-fit inclination angles were randomly distributed (see the green dashed histogram). However, we find a distribution peaked at $63^{\circ}$ and $70^{\circ}$. This can also be seen in Figure 30, where we compare our observed cumulative distribution of inclination angles to that of randomly distributed ones. Our distribution shows a deficit at inclination angles below $60^{\circ}$ and is just slightly higher at large inclination angles. A K-S test of the two distributions yields a $5.6 \%$ chance that they are drawn from the same parent distribution.

To examine whether the distribution of envelope parameters changes with inclination angle (which could imply a degeneracy), Figure 31 shows the reference envelope density $\rho_{1000}$, the total luminosity, and the cavity opening angle binned by three ranges of inclination angles. None of the three model parameters show a significantly different distribution for any of the inclination bins (K-S test significance levels are $\gtrsim 0.1$, except for the cavity opening angles for the lowest and middle inclination range, for which the K-S test significance value is 0.02). The median $\rho_{1000}$ values for the $i=18^{\circ}-41^{\circ}, 49^{\circ}-63^{\circ}$, and $69^{\circ}-87^{\circ}$ inclination bins are all $5.9 \times 10^{-19} \mathrm{~g} \mathrm{~cm}^{-3}$. Even though not shown in Figure 31, the objects whose best-fit model does not include an envelope are only found at $i \geqslant 49^{\circ}$. It is noteworthy that protostars with the highest envelope densities do not have inclination angles in the $69^{\circ}-87^{\circ}$ range; it is not clear whether this is an observational bias, whether our observed sample does not contain high-density, edge-on protostars, or whether this is due to biases in the fitting procedure and/or model grid. The median values for the total luminosity do not differ by much for the different bins of inclination angle, increasing from 2.9 to $4.1 L_{\odot}$ from the lowest to the middle inclination range and then decreasing to $2.0 L_{\odot}$ for the highest inclination angles. The few protostars with very high $L_{\text {tot }}$ values have large inclination angles $\left(i \geqslant 49^{\circ}\right)$. Finally, the distribution of cavity opening angles is quite similar for different ranges in inclination, except for a somewhat larger number of $\theta=45^{\circ}$ values at intermediate inclination angles. Half the objects in the $i=18^{\circ}-41^{\circ}$ and $69^{\circ}-87^{\circ}$ inclination bins have $\theta \leqslant 15^{\circ}$ (with the most common value $5^{\circ}$ ), while almost half the objects at intermediate inclination angles have $\theta \geqslant 35^{\circ}$ (the most common value is $45^{\circ}$ ).

In Figure 32, we show ratios of the total and bolometric luminosities as a function of inclination angle and foreground extinction ( $i$ and $A_{V}$ are adopted from the best model fits). The total luminosity is the intrinsic luminosity from the best-fit model of each object, while the bolometric luminosity is derived by integrating the fluxes of the observed SED. It is expected that $L_{\mathrm{tot}}$ is higher than $L_{\mathrm{bol}}$ for objects seen at higher 

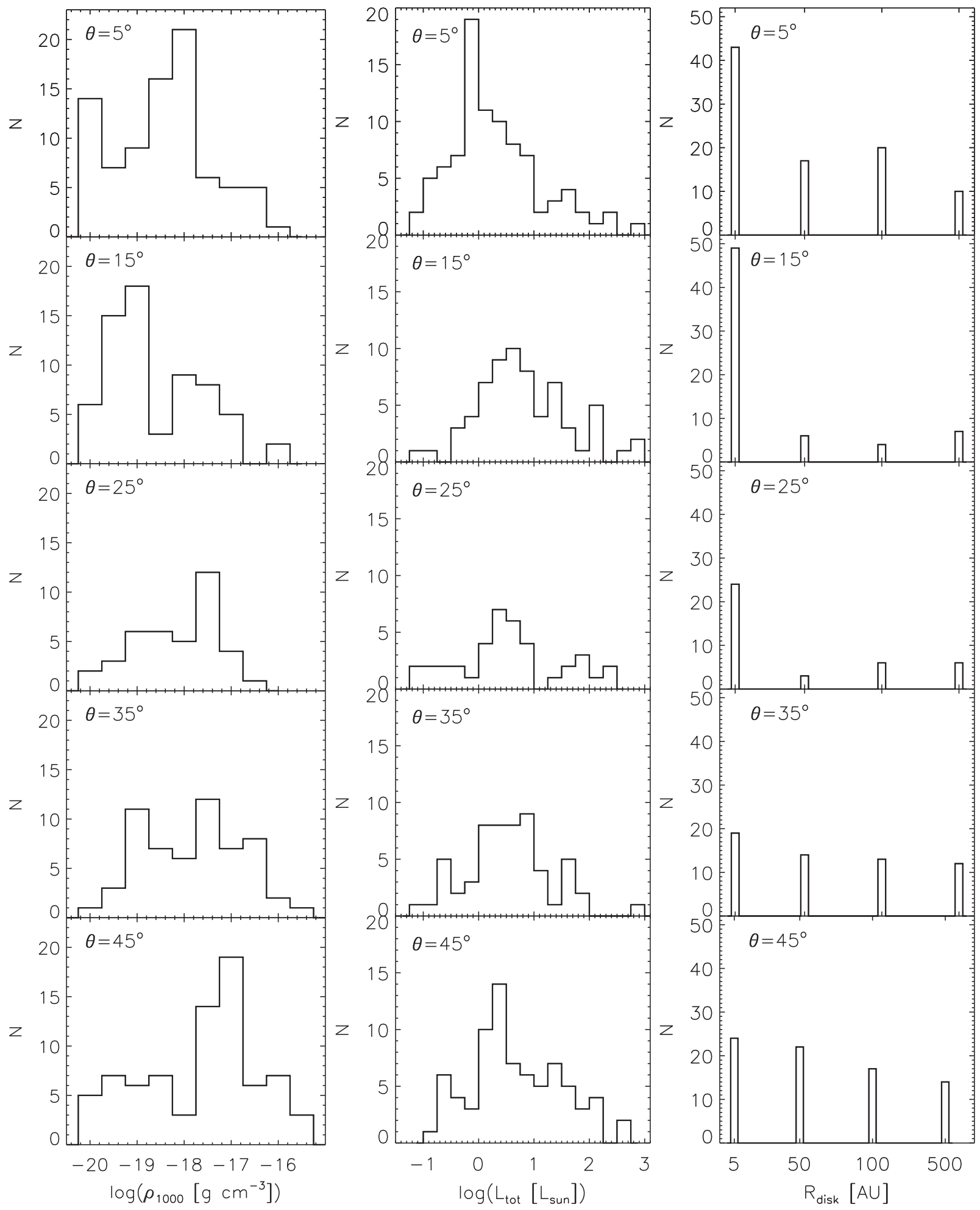

Figure 28. Histograms of the envelope reference density $\rho_{1000}$ (left), the total luminosity (middle), and the disk radius (right) of the best fits grouped by cavity opening angles.

inclination angles, since for these objects a large fraction of the emitted flux is not directed toward the observer (and thus deriving bolometric luminosities from observed fluxes will underestimate the intrinsic source luminosity). Conversely, objects seen more face-on should have lower $L_{\text {tot }}$ values compared to $L_{\mathrm{bol}}$. Our data and model fits yield $L_{\mathrm{tot}}$ values that are usually higher than the $L_{\mathrm{bol}}$ values measured from the SED; the discrepancy is larger for the more highly inclined sources.
The median $L_{\mathrm{tot}} / L_{\mathrm{bol}}$ ratio is 1.5 for protostars with inclination angles in the $18^{\circ}-41^{\circ}$ range, 2.5 for the $i=49^{\circ}-63^{\circ}$ range, and 3.5 for inclination angles $\geqslant 69^{\circ}$. The fact that $L_{\mathrm{tot}}>L_{\mathrm{bol}}$ even for $i=18^{\circ}-41^{\circ}$ could be related to the typically smaller cavity opening angles for this range of inclination angles (see Figure 31); less flux, especially at shorter wavelengths, is detected since the opacity along the line of sight is still high owing to the small cavities. 


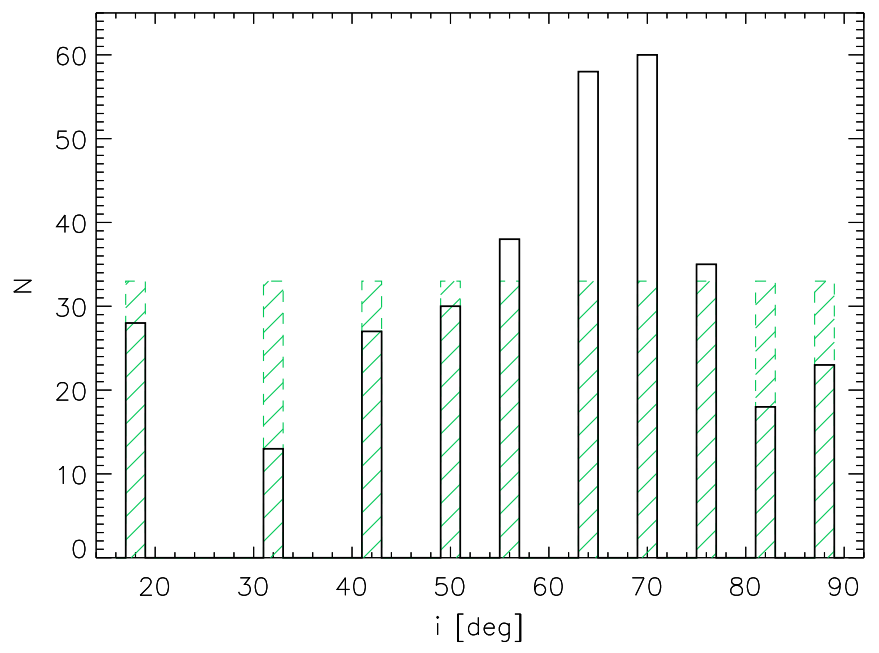

Figure 29. Histogram of the inclination angles of the best fits for the 330 targets in our sample. The green dashed histogram represents the distribution of uniformly (randomly) distributed inclination angles.

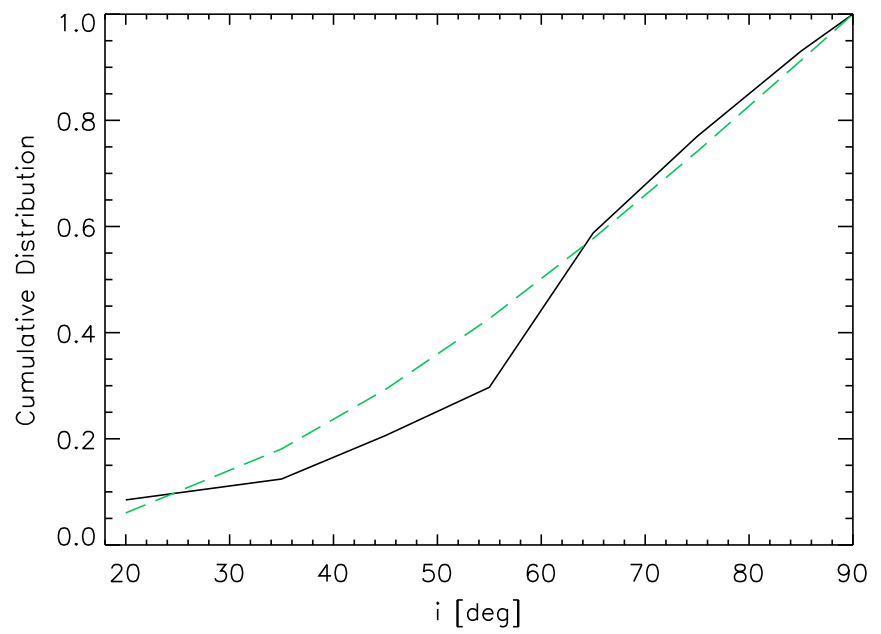

Figure 30. Cumulative distribution of the inclination angles of the best fits, normalized by the total number of fits (solid line), compared to the cumulative probability of finding an inclination angle below a given value for randomly distributed inclinations (green dashed line).
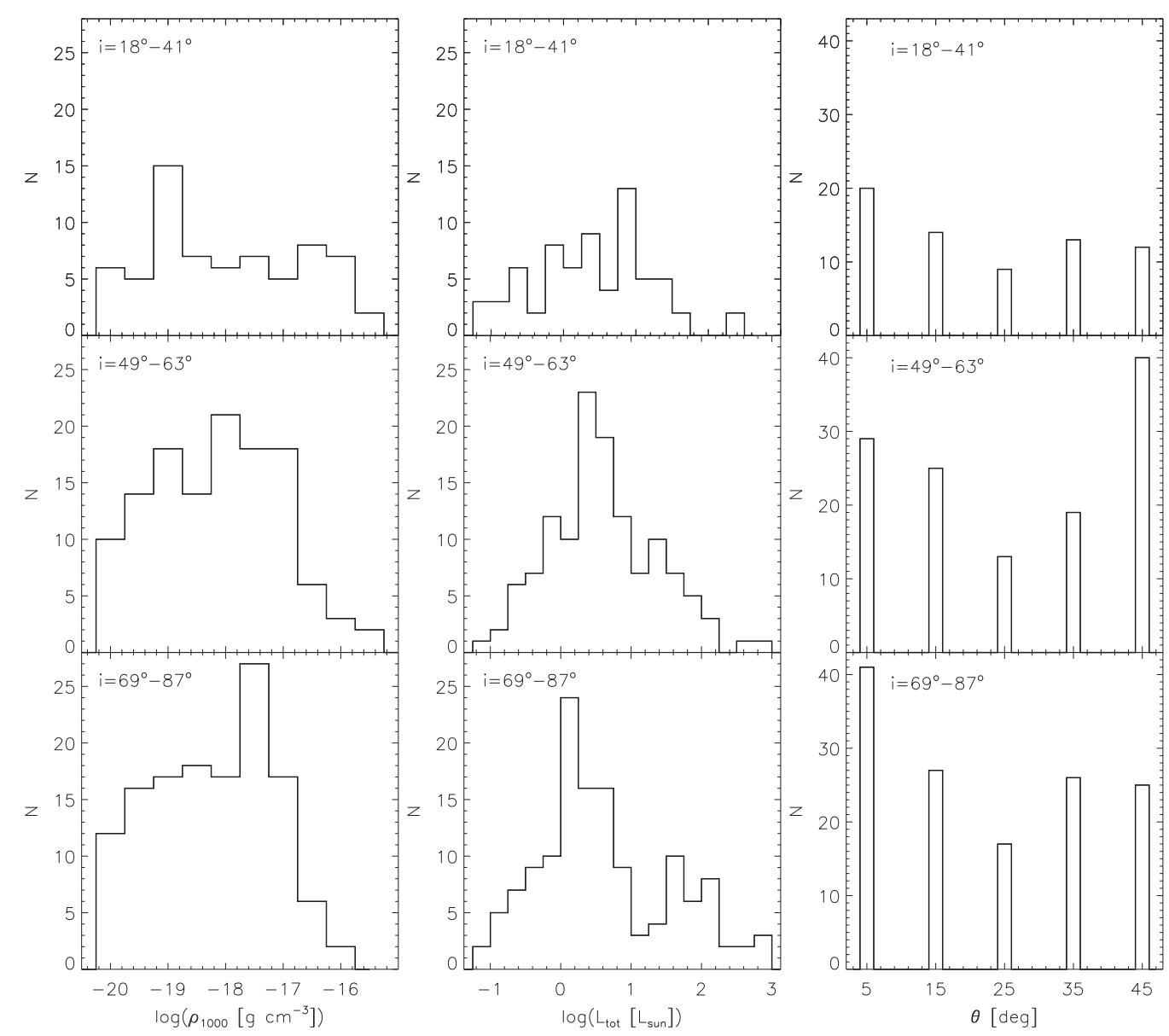

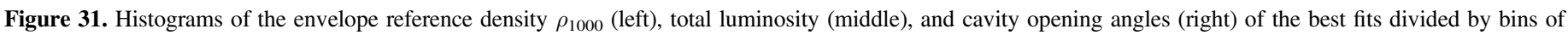
inclination angles.

Foreground extinction also plays a role in increasing the $L_{\mathrm{tot}} / L_{\mathrm{bol}}$ ratio. The median ratio of these luminosities increases from 1.8 for the $A_{V}=0-5 \mathrm{mag}$ range to 5.0 for $A_{V}=25-30$; it decreases somewhat for the next $A_{V}$ bin, but reaches 5.9 at $A_{V}=40-50$ (the 23 objects with $A_{V}>50$, not shown in
Figure 32, have a median $L_{\mathrm{tot}} / L_{\mathrm{bol}}$ ratio of 8.2). Among the 22 objects with best-fit $A_{V}$ values of $0-5 \mathrm{mag}$ and inclination angles $\leqslant 50^{\circ}$, only four have $L_{\mathrm{tot}} / L_{\mathrm{bol}}$ ratios that are larger than 1.5 (they are HOPS 57, 147, 199, and 201; in most cases the model overestimates the near-IR emission). 


\subsection{Envelope Parameters for Different SED Classes}

Figures 33-38 divide the histograms of the best-fit reference density $\rho_{1000}$, inclination angle, cavity opening angle, total luminosity, disk radius, and foreground extinction, respectively, by protostar class. As explained in Section 3, we divided our targets into Class 0, Class I, flat-spectrum, and Class II objects based on their mid-infrared $(4.5-24 \mu \mathrm{m})$ spectral index and bolometric temperature (see also Table 1). Thus, Class 0 and I protostars have a spectral index $>0.3$, and Class 0 protostars have $T_{\text {bol }}$ values $<70 \mathrm{~K}$, but, as mentioned in Section 3 , there are a few protostars whose spectral index or $T_{\text {bol }}$ value places them very close to the transition region between Class 0 and I or between Class I and flat spectrum. Given that our sample contains just 11 Class II pre-mainsequence stars, we did not include them in the following histograms; they will be discussed in Section 7.2.3.

The distributions of reference densities (Figure 33) are different for all SED classes; none are consistent with being drawn from the same parent population $(\mathrm{K}-\mathrm{S}$ test significance level $<0.01)$. Overall, Class 0 protostars have higher envelope densities than Class I and flat-spectrum sources; the median $\rho_{1000}$ values decrease from $5.9 \times 10^{-18} \mathrm{~g} \mathrm{~cm}^{-3}$ to $2.4 \times 10^{-19} \mathrm{~g} \mathrm{~cm}^{-3}$ to $1.2 \times 10^{-19} \mathrm{~g} \mathrm{~cm}^{-3}$ for these three groups. The lower and upper quartiles for $\rho_{1000}$ are $1.8 \times 10^{-18}$ and $1.8 \times 10^{-17} \mathrm{~g} \mathrm{~cm}^{-3}$ for the Class 0 protostars and $2.4 \times 10^{-20}$ and $1.2 \times 10^{-18} \mathrm{~g} \mathrm{~cm}^{-3}$ for the Class I and flat-spectrum objects. We will discuss some implications of these differences in derived envelope densities in Section 7.2.

For the inclination angles (Figure 34), the distributions are significantly different for all protostellar classes, too (K-S test significance level $\ll 0.01)$. As was shown in Figure 29 , a random distribution of inclination angles would result in equal numbers of protostars at each value; there is a deficit of Class 0 and Class I protostars at $i \lesssim 60^{\circ}$, and there are also few Class I protostars and hardly any flat-spectrum sources at the highest inclination angles. The median inclination angle is highest for Class 0 protostars $\left(70^{\circ}\right)$ and then decreases somewhat for Class I protostars $\left(63^{\circ}\right)$ and even more for flat-spectrum sources $\left(57^{\circ}\right)$. Similar to the envelope density, the median inclination angle decreases as one progresses from Class 0 to flat-spectrum sources.

In the distributions of cavity opening angles (Figure 35), significant differences can be found between Class 0 and Class I protostars and between Class I protostars and flat-spectrum sources (K-S test significance level $\ll 0.01)$. The median cavity opening angle is $15^{\circ}$ for the Class I protostars, but $25^{\circ}$ for the other two classes. About $40 \%$ of Class I protostars have $\theta=5^{\circ}$, while the distribution among the different cavity opening angles is flatter for the other two object classes. The large fraction of Class I protostars with small cavities could be the result of degeneracy in model parameters (see Section 7.2) or our assumptions on envelope geometry (see Section 7.4). There are notably few flat-spectrum sources with a $5^{\circ}$ cavity opening angle; most of them have cavity opening angles of $15^{\circ}$ or $45^{\circ}$.

When comparing the total luminosities for the different SED classes (Figure 36), the distribution of $L_{\mathrm{tot}}$ values is different for the Class 0 protostars when compared to the other two classes (K-S test significance level $<0.015$ ), but similar for Class I protostars and flat-spectrum sources. The median total luminosity for Class 0 protostars is $5.5 L_{\odot}$, compared to $2.0 L_{\odot}$ for Class I protostars and $3.0 L_{\odot}$ for flat-spectrum sources. Both Class 0 and I protostars cover close to the whole range of $L_{\text {tot }}$ values in the model grid $\left(\sim 0.06-600 L_{\odot}\right)$, while flat-spectrum sources span a more limited range, from 0.1 to $316 L_{\odot}$.

The distribution of centrifugal radii for the whole sample showed a preference for 5 au (see Figure 26). When separating the best-fit disk radii by protostellar class (Figure 37), it is clear that the trend for small centrifugal radii is driven by the flatspectrum sources and also Class I protostars. The fraction of Class 0 protostars with $R_{\text {disk }}=5$ au is $17 \%$; it increases to $46 \%$ and $73 \%$ for Class I protostars and flat-spectrum sources, respectively. The median disk radius decreases from 100 au for Class 0 protostars to 50 au for Class I protostars to 5 au for flatspectrum sources. All three histograms are significantly different from one another (K-S test significance level $\ll 0.001)$. The unexpectedly small centrifugal radii for Class I protostars and flat-spectrum sources could point to parameter degeneracies (see Section 7.2) or the need to revise certain model assumptions (see Section 7.4).

Finally, the distribution of best-fit foreground extinction values (Figure 38) is similar for all three object classes (K-S test significance level $>0.03$ ). Even the median values are close: $A_{V}=9.2$ for Class 0 protostars, $A_{V}=8.9$ for Class I protostars, and $A_{V}=10.1$ for flat-spectrum sources. Most objects are fit with relatively low foreground extinction values. As can be seen from Figure 39, the majority of protostars have best-fit $A_{V}$ values well below the maximum $A_{V}$ values determined from column density maps, which were used as the largest allowed $A_{V}$ values for the SED fitter. The ratio of model-derived $A_{V}$ to observationally constrained maximum $A_{V}$ is lower than 0.5 for about $60 \%$ of the sample.

In Figure 40, we plot the reference densities $\rho_{1000}$ versus the foreground extinction for Class 0, Class I, and flat-spectrum sources. As was already seen in Figure 38, the extinction along the line of sight is similar for all three classes, with most objects in the $A_{V} \sim 0-30$ regime. Class 0 protostars, which have higher envelope densities, tend to have lower $A_{V}$ values from foreground extinction; the highest-density envelopes are spread among a wide range of $A_{V}$ values. The result is similar for Class I protostars. Flat-spectrum sources display a range in envelope densities at various foreground extinction values; the lowestdensity envelopes typically have $A_{V}<20$. Thus, foreground extinction does not seem to affect the classification of protostars. This result is also supported by the statistical analysis of Stutz \& Kainulainen (2015), who found that, for $A_{V}$ values up to 35 , the misclassification of a Class I protostar as a Class 0 protostar due to foreground extinction (which results in a lower $T_{\text {bol }}$ ) is low.

We found differences in the best-fit envelope densities and inclination angles for the various protostellar classes. The result that Class 0 protostars tend to have larger inclination angles and envelope densities compared to Class I and flat-spectrum objects can also be seen in Figure 41. There are very few Class 0 protostars with low inclination angles; most have relatively high density and $i>60^{\circ}$. Class I protostars are best fit by somewhat lower inclination angles than Class 0 protostars and also lower $\rho_{1000}$ values. The best-fit reference density for Class I protostars decreases as the inclination angle increases; thus, higher-density protostars are typically classified as Class I protostars only if they are not seen at close to edge-on orientations. Flat-spectrum sources are spread out in densityinclination space, but intermediate inclination angles and low envelope densities are common. There is a relatively large 

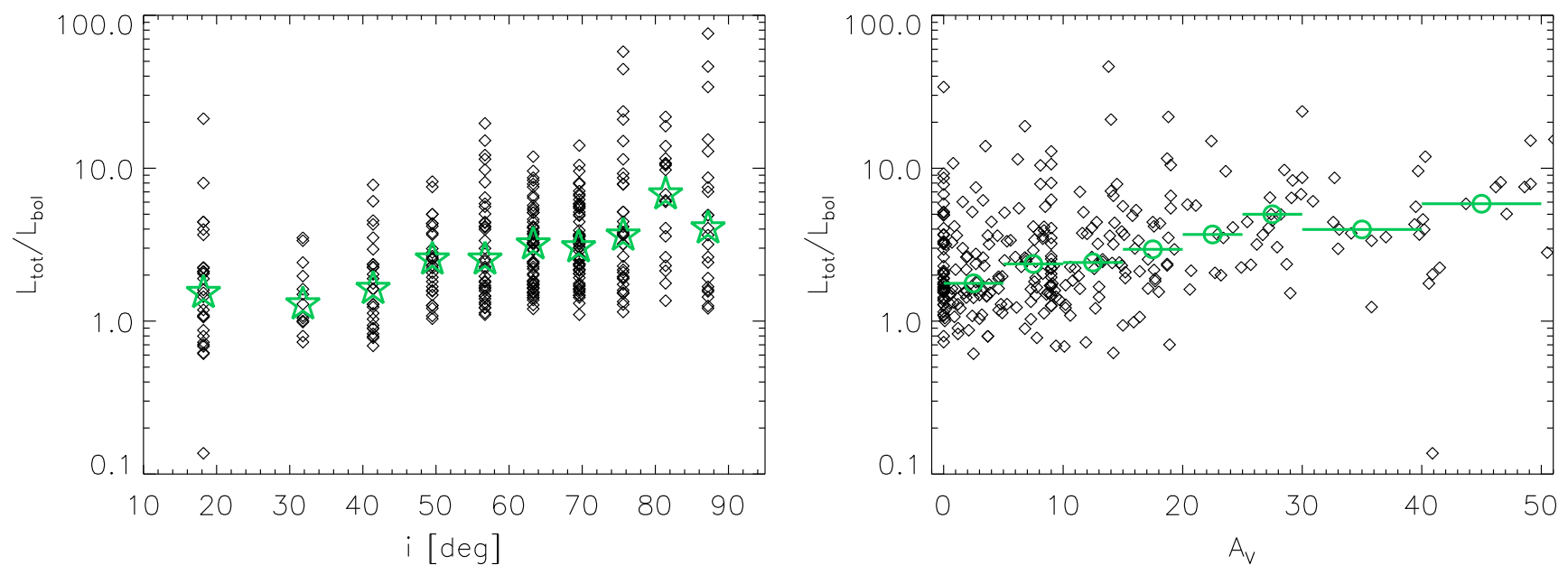

Figure 32. Ratio of the total luminosity from the best fits and the bolometric luminosity derived from the observed SEDs vs. the inclination angle (left) and foreground extinction (right) of the best fits. In the left panel, the open stars represent the median ratios at each inclination angle. In the right panel, the open circles represent the median ratios for eight bins in $A_{V}$ values, represented by the horizontal lines bisecting each circle.

number of objects at $i=18^{\circ}$ and a deficit of objects at high inclination angles. The highest-density flat-spectrum sources are seen at inclination angles $<50^{\circ}$, while the lower-density objects cover almost the full range of inclination angles.

The median parameter values we determined from the best fits for the Class 0, Class I, and flat-spectrum sources (see Table 4) can be used to show representative median SEDs for each protostellar class. In Figure 42, we show model SEDs whose parameter values are equal to the median values found for each of the three protostellar classes. It is apparent that the large envelope density and higher inclination angle for Class 0 protostars cause a deep absorption feature at $10 \mu \mathrm{m}$ and a steeply rising SED in the mid- and far-IR, with a peak close to $100 \mu \mathrm{m}$. In Class I protostars, the SED is less steep and peaks at a shorter wavelength than the median SED of Class 0 protostars. Flat-spectrum sources show the strongest near-IR emission of the three protostellar classes; their median SED is very flat out to $70 \mu \mathrm{m}$, but at longer wavelengths it is very similar in shape and flux level to that of Class I protostars.

\subsection{Estimating Parameter Uncertainties}

Given that the $R$ values are a measure of the goodness of fit in units of the fractional uncertainty, we can use models that lie within a certain range of the best-fit $R$ value to estimate uncertainties for the various model parameters. For each modeled HOPS target, we tabulated the model parameters for all those models that lie within a difference of $0.5,1.0,1.5$, and 2.0 of the best-fit $R$. We then computed the mode (i.e., the value with the highest frequency) for the inclination angle, total luminosity, $\rho_{1000}$, cavity opening angle, outer disk radius, and $A_{V}$ in each of the $\Delta R$ bins for each object. For any given protostar, the models in each $\Delta R$ bin span certain ranges in parameter values; while the modes do not capture the full extent of these ranges, they convey the most common value within each parameter range. The farther away a mode is from the best-fit value, the more poorly constrained the model parameter. Conversely, if a mode of a certain parameter is close to or matches the best-fit value, especially for $\Delta R=1.5$ or 2 , that particular model parameter is well-constrained. In Figures 43-48 we plot the mode versus the best-fit value for six model parameters and four $\Delta R$ bins for all 330 targets in our sample. The larger $\Delta R$, the larger the spread in modes is expected to be for each parameter value.

For example, Figure 43 shows that even when considering all models with an $R$ value of up to 2 larger than the best-fit $R$ $(\Delta R=2)$, the inclination angle for objects with a best-fit $i$ of $18^{\circ}$ is well-constrained; most modes lie at $i=18^{\circ}$, too, and only a few modes can be found at larger inclination angles. However, objects with best-fit $i$ values of $32^{\circ}$ or $41^{\circ}$ typically can also be fit by models with lower inclination angles (the majority of modes lies below the line where mode and best-fit value are equal). Inclination angles $\gtrsim 63^{\circ}$ are better constrained, since their modes mostly lie at high inclination angle values, but there are protostars with modes of $i=18^{\circ}$, too.

The modes for the total luminosity (Figure 44) show a small spread for models within $\Delta R=0.5$, but the spread increases as $R$ increases, with some objects displaying up to an order of magnitude in variation of $L_{\text {tot }}$. As illustrated in Figure 45, the reference density $\rho_{1000}$ is usually well-constrained; however, as $R$ increases, the modes of the $\rho_{1000}$ values are often lower than the best-fit values. For the cavity opening angle (Figure 46), many models up to $\Delta R=2$ have modes of $\theta=45^{\circ}$, independent of the best-fit value. Similarly for the centrifugal radius (Figure 47), $R_{\text {disk }}=500$ au is a common value. For all four disk radii, the modes tend to be larger than the best-fit values; in particular, objects with a best-fit $R_{\text {disk }}$ of 5 au have a very uncertain disk radius. In general, it looks like our models do not constrain the disk radius and cavity opening angle well. The foreground extinction (Figure 48) displays a certain range of modes for each best-fit value, but objects with $A_{V} \lesssim 20$ typically have more reliable $A_{V}$ values from their model fits.

Figures 43-48 allow us to gauge general trends between best-fit values and modes for different model parameters. For results on individual objects, we refer to Appendix B, where we show plots of the difference between the modes and the best-fit values of the major model parameters for all modeled HOPS targets. In this way it is possible to estimate which models are better constrained and thus which objects have more reliable SED fits. In addition, in Appendix B we also include contour plots of $R$ values for different pairs of model parameters for a few targets to illustrate typical parameter degeneracies, which also contribute to parameter uncertainties. 


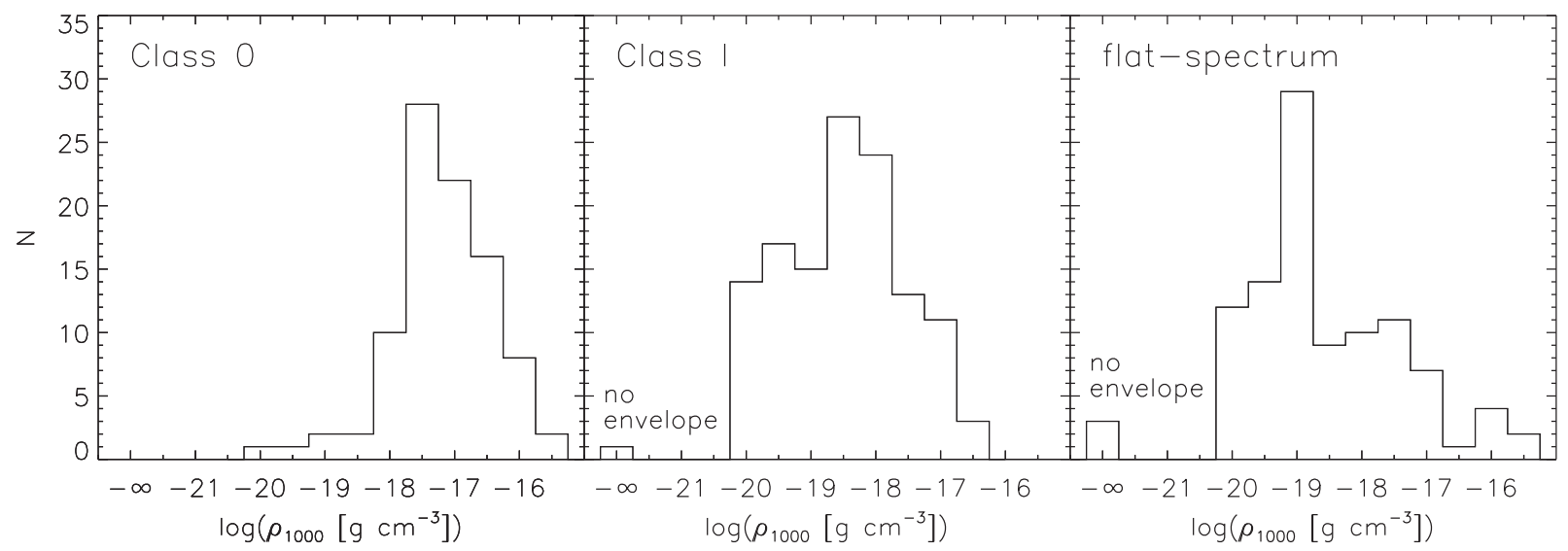

Figure 33. Histograms of the envelope reference density $\rho_{1000}$ of the best fits for the different SED classes.

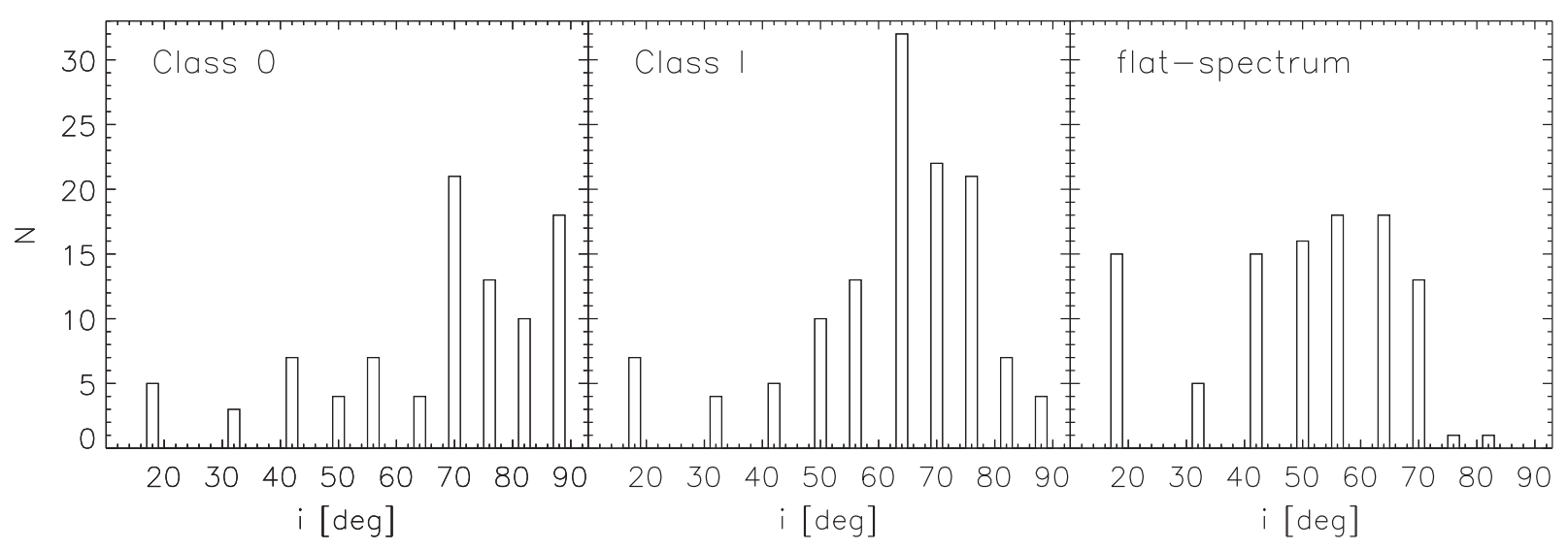

Figure 34. Histograms of the inclination angles of the best fits for the different SED classes.

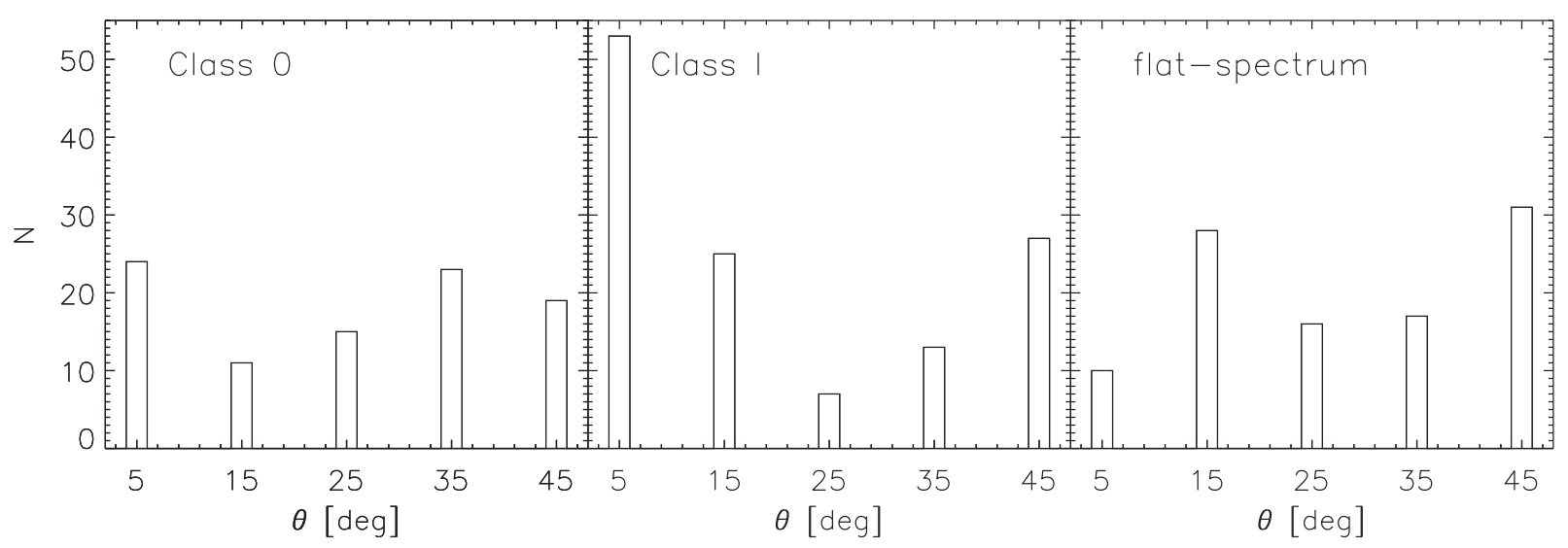

Figure 35. Histograms of the cavity opening angles of the best fits for the different SED classes.

\section{DISCUSSION}

\subsection{Deriving Envelope Parameters from a Model Grid}

We compared a grid of TSC models to each target by ranking the models using a statistic, $R$, which measures the deviation between observed and model fluxes in logarithmic space. We did not model each source by further adjusting the model parameters, but instead identified the best-fit SED from our model grid. Thus, we are bound by the range and sampling of parameters chosen for the grid, and while we constructed the grid with the aim of covering the typical parameter space for protostars, it is limited to discrete values. It is likely that many protostars have best-fit parameters that would fall between those sampled by the grid, and a few objects could have parameter values that lie beyond the limits set by the grid. In addition, TSC models are axisymmetric and have mostly smooth density and temperature profiles, and they do not include external heating. They assume a rotating, infalling envelope with constant infall rate and with the gravitational force dominated by the central protostar, but the true envelope structure is likely more complex. The models would not apply to the collapse of a cloud in an initial filamentary or sheet-like 


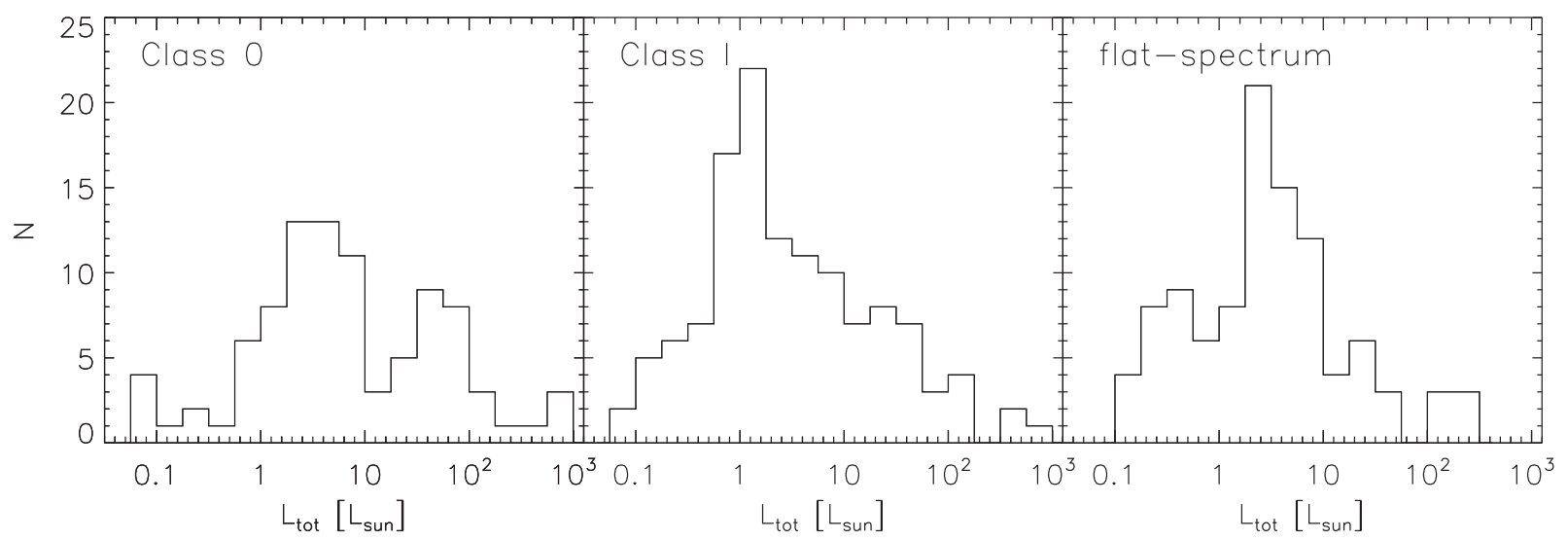

Figure 36. Histograms of the total luminosity of the best fits for the different SED classes.

geometry or to multiple systems with, e.g., more than one outflow cavity (e.g., Hartmann et al. 1996; Tobin et al. 2012).

Despite the relatively simple models that we use, many of the observed SEDs are fit remarkably well: $75 \%$ of the fits have $R<4$. In those cases, the continuum traced by the IRS spectrum, the silicate absorption feature at $10 \mu \mathrm{m}$, and the PACS fluxes are all accurately reproduced by the model. Even many flat-spectrum sources, which often do not display any spectral features in the mid-infrared and have an overall flat SED out to 30 or $70 \mu \mathrm{m}$, often have models that fit them very well. About $75 \%$ of Class I protostars and $\sim 70 \%$ of Class 0 protostars have $R<4$, while close to $90 \%$ of flat-spectrum sources have $R$ values in this range. This validates the choice of parameter values for our model grid. Additional constraints, like limits on foreground extinction or information on the inclination and cavity opening angles from scattered light images or mapping of outflows, would allow us to further test and refine the models. We have used limits on the extinction in our analysis. Although Hubble Space Telescope (HST) scattered light images have been used to constrain models for one HOPS protostar (Fischer et al. 2014), scattered light images are not available for many of our targets. We therefore chose to focus on fitting the SEDs of all of our targets in a uniform way to a well-defined set of models. Future studies will incorporate scattered light images and compare the results to those from the SED fits (J. Booker et al. 2016, in preparation).

The best-fit models from our grid for the HOPS targets both reproduce the SEDs and yield estimates for their protostellar parameters, mostly envelope properties. However, these are not necessarily unique fits to the data for three reasons. First, there are degeneracies in the model parameters; increasing the envelope density or inclination angle, or decreasing the cavity opening angle or disk radius, results in a steeper mid-IR SED slope and deeper silicate features. Each of these parameters affects the SED differently (just the general trends are the same), and the best fit for each object tries optimizing them. The next best fit, however, could be a different combination of these parameters, especially if the SED is not well-constrained by observations (see Section 6.4). Second, although the TSC models reproduce the observed SEDs, other models with different envelope geometries may also be able to fit the same SEDs. The modeling presented here is only valid in the context of the TSC models of single stars, and the resulting derived properties are only valid within that framework. Last, neglecting external heating could result in overestimated envelope densities and luminosities, with the most noticeable effects ( $\rho_{1000}$ and $L_{\text {tot }}$ too large by factors of a few) on lowluminosity sources exposed to strong radiation fields (see Section 4.3). From the distribution of best-fit $L_{\text {tot }}$ values, we estimate that $\sim 20 \%$ of HOPS targets in our sample could be affected by external heating. Even though we do not know the strength of external heating for each protostar, it is likely that external heating would only result in relatively small changes in the derived envelope parameters for these protostars.

\subsection{Envelope Properties and SED Classes}

When comparing envelope parameters sorted by SED classes, we found that envelope densities and inclination angles decrease from the sample of Class 0 protostars through that of Class I protostars to that of flat-spectrum objects. The former is likely an evolutionary effect, while the latter confirms the results of previous work (e.g., Evans et al. 2009) that the inclination angle has an important effect on the SED and that the evolutionary state of an object cannot be derived from SED slopes alone. Thus, there is a difference between the "stage" and "class" of an object (Robitaille et al. 2006); Stage 0 and I objects are characterized by substantial envelopes, Stage II objects are surrounded by optically thick disks, with possibly some remnant infalling envelopes, and Stage III objects have optically thin disks.

In general, the trends we see among model parameters are a consequence of the definition of a protostar based on its SED: in order to be classified as a Class 0 or I object, a protostar is required to have a near- to mid-infrared SED slope larger than 0.3 . A protostellar model with a small cavity opening angle, small centrifugal radius, and/or high inclination angle will generate such an SED, since it increases the optical depth along the line of sight. Models with a large cavity will only yield a rising SED in the $2-40 \mu \mathrm{m}$ spectral range if their envelope density is large or the inclination angle is relatively high.

We find that Class 0 protostars can be best fit not only by very high envelope densities but also moderately high envelope densities and large inclination angles. The bolometric temperature, $T_{\text {bol }}$, which is used to separate Class 0 from Class I protostars, is inclination dependent; some Class I protostars are shifted to Class 0 protostars if they are viewed more edge-on. The higher-density Class I protostars tend to have lower inclination angles (but still $>50^{\circ}$ ); thus, their evolutionary stage could be similar to more embedded protostars that are seen edge-on and classified as Class 0 protostars. Conversely, some Class 0 protostars with large inclination angles, but lower 


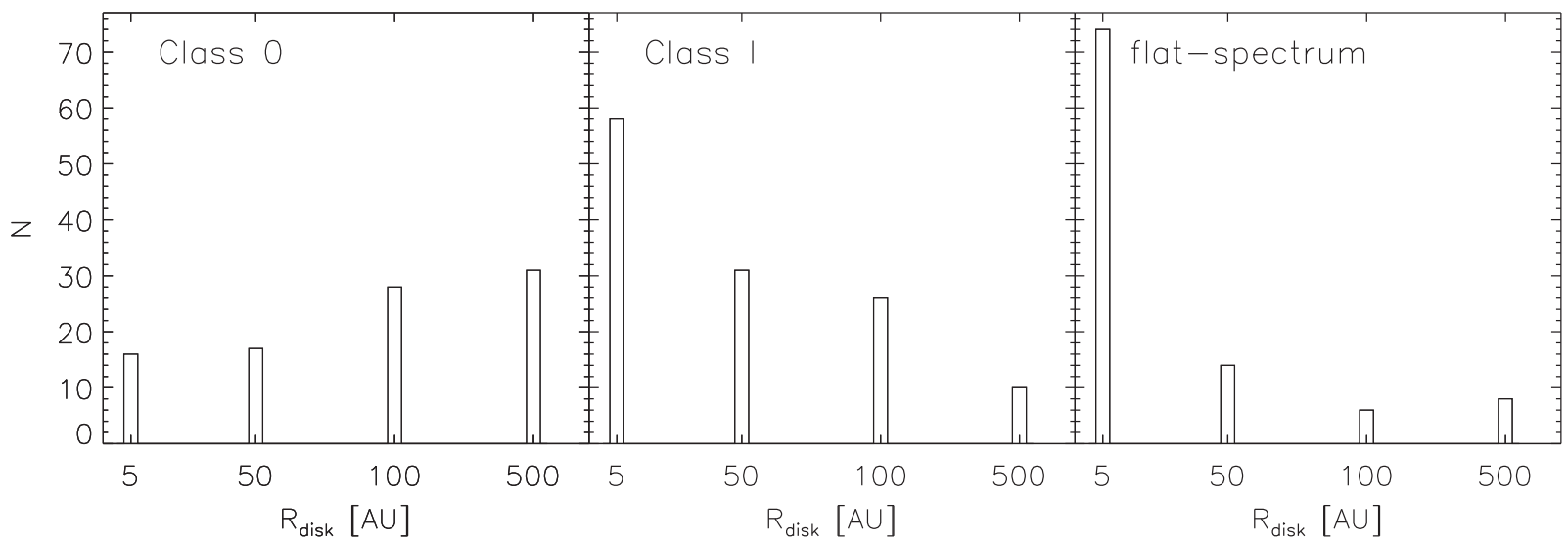

Figure 37. Histograms of the disk radii of the best fits for the different SED classes.

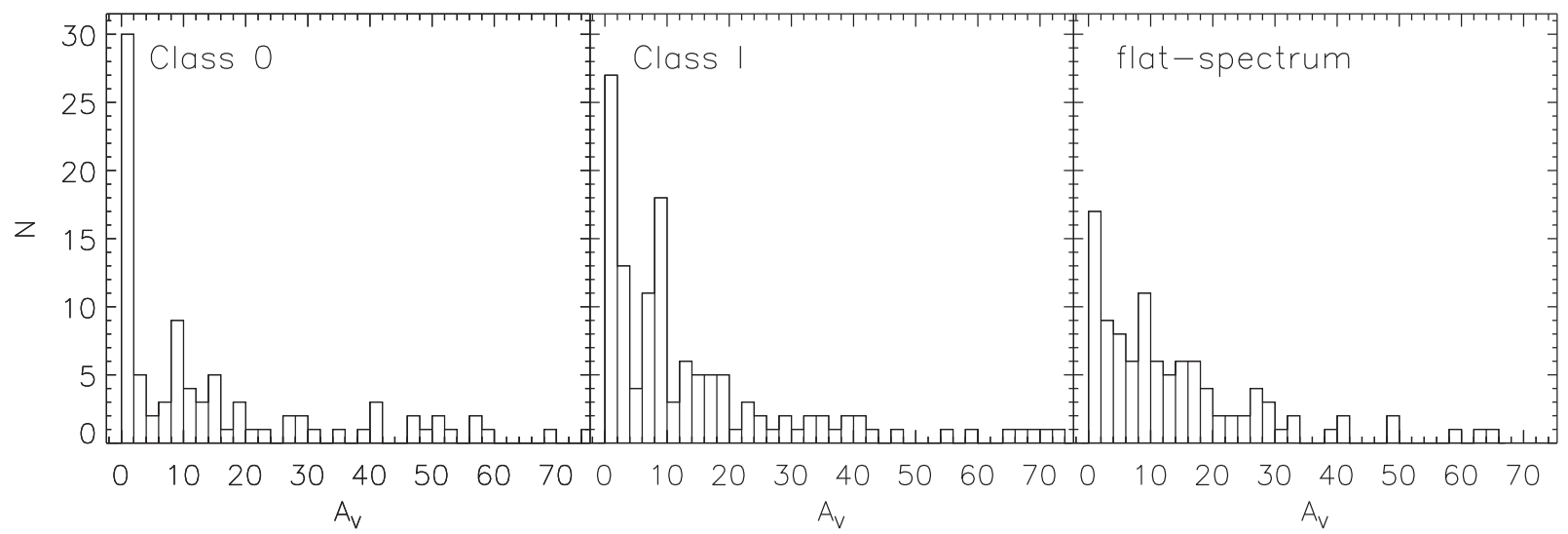

Figure 38. Histograms of the foreground extinction of the best fits for the different SED classes.

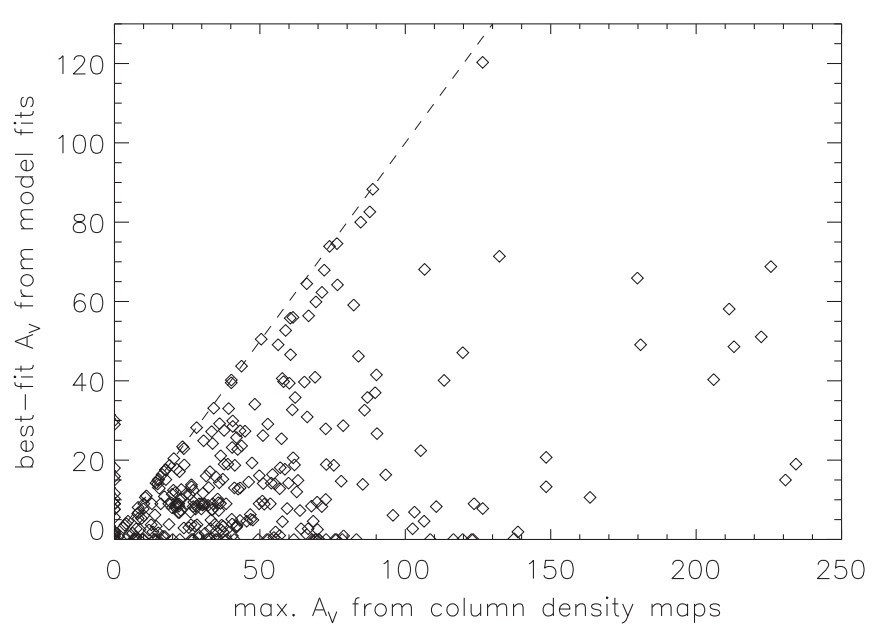

Figure 39. Foreground extinction values $A_{V}$ from the best-fit models vs. the maximum $A_{V}$ value determined from column density maps of Orion. The dashed line indicates where the two $A_{V}$ values are equal.

envelope densities, could be in a later evolutionary stage than typical Class 0 protostars. Similarly, Class I protostars with large $i$ and low $\rho_{1000}$ values could be edge-on Stage II objects (whose infrared emission is dominated by a disk). Finally, lowinclination Stage 0 and Stage I protostars can appear as flatspectrum sources (Calvet et al. 1994).

Nevertheless, the observed trend in envelope densities suggests that the variations in the observed SEDs track, in great part, an evolution toward lower envelope densities and lower infall rates. Assuming a certain mass for the central star, the reference density in our models can be used to infer an envelope infall rate $\left(\dot{M}_{\text {env }} \propto \rho_{1000} \sqrt{M_{*}}\right)$. As mentioned in Section 4.1 , this infall rate is model dependent and therefore tied to the assumptions of the models. With this in mind, the median $\rho_{1000}$ values for the Class 0 , Class I, and flat-spectrum protostars in our sample correspond to envelope infall rates of $2.5 \times 10^{-5}, 1.0 \times 10^{-6}$, and $5.0 \times 10^{-7} M_{\odot} \mathrm{yr}^{-1}$, respectively, for a $0.5 M_{\odot}$ star. Using a more realistic assumption of larger stellar mass for more evolved protostars, the infall rates for Class I and flat-spectrum protostars would be larger than these values by a factor of a few. However, just larger stellar masses cannot explain the large decrease of a factor of 50 in the median envelope density from Class 0 to flatspectrum protostars; to achieve such a decrease with a constant infall rate of $2.5 \times 10^{-5} M_{\odot} \mathrm{yr}^{-1}$, the stellar mass would have to increase by a factor of 2500 . Thus, within the context of our model fits, we can conclude that, as envelopes become more tenuous, the infall rates also decrease.

Other trends are also apparent. Class 0 protostars and flatspectrum sources show a relatively flat distribution of cavity opening angles. On the other hand, the best fit for a large fraction of Class I protostars $(40 \%)$ results in $\theta=5^{\circ}$. This could point to a degeneracy in the models, since protostars with small cavity opening angles tend to have lower envelope densities (and also lower total luminosities); thus, the smaller cavity partly compensates for the lower opacity resulting from the lower envelope density (see also Figure 49). 


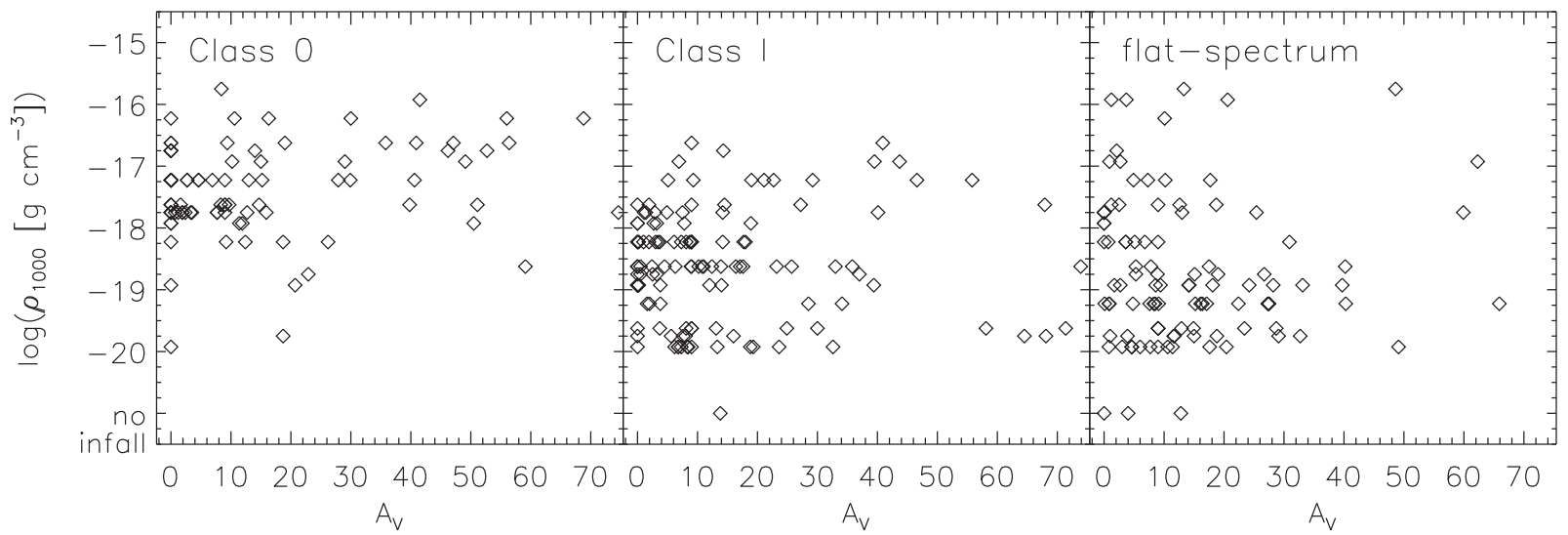

Figure 40. Best-fit $\rho_{1000}$ values vs. the foreground extinction for the different SED classes. Note that there are a few objects at $A_{V}>75$, but they are not shown for overall clarity of the figure.

Even though our models do not yield reliable disk properties, we can make a statement about the difference in the best-fit centrifugal radii (or $R_{\text {disk }}$ ), which are tied to the structure of the rotating envelope given by the model fits. It should be noted that the centrifugal radii set a lower limit to the disk radii, since disks may spread outward owing to viscous accretion. Most Class I protostars and flat-spectrum sources are fit with a centrifugal radius of just $5 \mathrm{au}$. Since the smallest centrifugal radius in our model grid is $5 \mathrm{au}$ and the next value is $50 \mathrm{au}$, we can state that, except for Class 0 protostars, most protostars in our sample have $R_{\text {disk }}<50$ au, and some might even have $R_{\text {disk }}<5$ au.

Small disks of those sizes have been observed; radio interferometry of the multiple protostellar system L1551 IRS 5 shows disks whose semimajor axes are $\lesssim 20$ au (Rodríguez et al. 1998; Lim \& Takakuwa 2006). However, there is a degeneracy between the centrifugal radius and the envelope density; for protostars with low envelope densities, the small centrifugal radius can compensate the decrease in opacity by concentrating more material closer to the star. As can be seen in Figure 50, most protostars with $R_{\text {disk }}=5$ au also have lower envelope densities. Inclination angle also plays a role; protostars seen at $i>80^{\circ}$ typically have larger centrifugal radii. In addition, our envelope models include outflow cavities, which allow some of the mid-IR radiation to escape. In order to generate model SEDs with silicate absorption features and steep mid-IR slopes at low to intermediate inclination angles, a lower $R_{\text {disk }}$ value is needed. We will discuss the potential implications of the small cavity sizes and $R_{c}$ values for Class I protostars and flat-spectrum sources in Section 7.4.

\subsubsection{The Most Embedded Protostars}

Among the Class 0 protostars, there are protostars in the earliest evolutionary stages, when the envelope is massive and the protostar still has to accrete most of its mass. Stutz et al. (2013) identified 18 protostars with very red mid- to farinfrared colors $\left(\log \left(\lambda F_{\lambda}(70) / \lambda F_{\lambda}(24)\right)>1.65\right)$, of which 11 were newly identified (see Table 6). Tobin et al. (2015) added an additional object. These protostars were named PACS Bright Red sources (PBRs) by Stutz et al. (2013); they are HOPS 169, 341, 354, 358, 359, 372, 373, 394, 397-405, 407, and 409. Based on their steep 24-70 $\mu \mathrm{m}$ SEDs and large submillimeter luminosities, they were interpreted as the youngest protostars in Orion with very dense envelopes.

From our best-fit models to the SEDs of the PBRs, we derive a median $\rho_{1000}$ value of $1.2 \times 10^{-17} \mathrm{~g} \mathrm{~cm}^{-3}$, which is twice as high as the median value of all the Class 0 protostars in our sample. These fits also result in a median envelope mass within 2500 au of $0.66 M_{\odot}$ for the PBRs, but the individual objects cover a large range, from 0.07 to $1.83 M_{\odot}$. The median total luminosity amounts to $5.6 L_{\odot}$ (with a range from 0.6 to $\left.71.0 L_{\odot}\right)$, which is very similar to the median $L_{\text {tot }}$ value for the Class 0 protostars in our sample. Most PBRs (14 out of 19 protostars) are fit by models with large inclination angles $\left(i \geqslant 70^{\circ}\right)$, but, as shown in Stutz et al. (2013), high inclination alone cannot explain the redness of the PBRs. Thus, our models confirm the results of Stutz et al. (2013) that the PBRs are deeply embedded and thus likely among the youngest protostars in Orion.

\subsubsection{Flat-spectrum Sources}

A particularly interesting group of protostars that are not easy to categorize are the flat-spectrum sources. They are thought to include objects in transition between Stages I and II, when the envelope is being dispersed (Greene et al. 1994). In particular those with low envelope densities could be more evolved protostars, or they could be protostars that started out with more tenuous envelopes. On the other hand, flat-spectrum sources could also be highly inclined disk sources (see Crapsi et al. 2008), or protostars surrounded by dense envelopes, but seen close to face-on (Calvet et al. 1994). This type of misclassification could have a large effect on the lifetimes of the earlier protostellar stages and thus on the timeline of envelope dispersal. Among the 330 HOPS targets in our sample, we identified 102 flat-spectrum sources based on their flat $(-0.3$ to +0.3$)$ spectral index from 4.5 to $24 \mu \mathrm{m}$ (or $5-25 \mu \mathrm{m}$ in a few cases). Thus, they compose a fairly large fraction of our protostellar sample. Of these 102 objects, 47 have a negative spectral index and 55 have one between 0 and $+0.3 ; 41$ have a spectral index between -0.1 and 0.1 , which results in a very flat mid-infrared SED.

Despite a flat SED slope between 4.5 and $24 \mu \mathrm{m}$, many flatspectrum sources display a weak silicate emission or absorption feature at $10 \mu \mathrm{m}$, which may indicate the presence of a very tenuous infalling envelope or may be the result of the viewing 


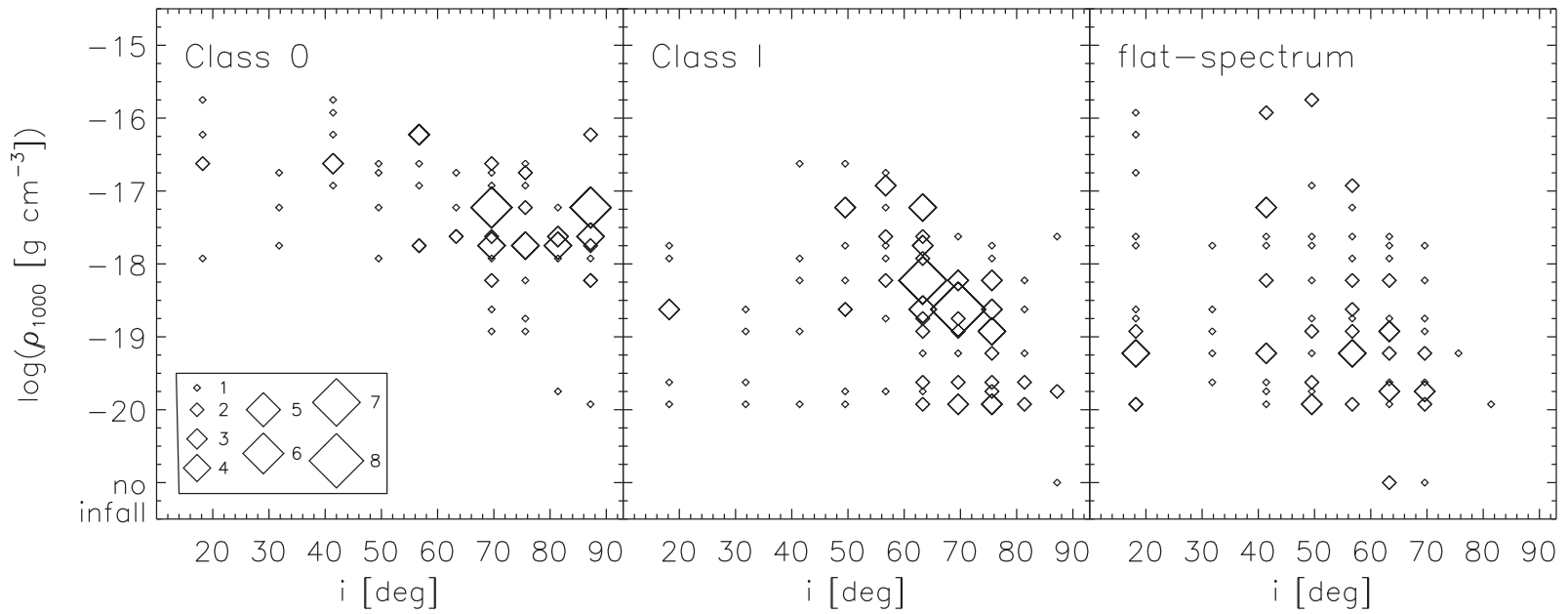

Figure 41. Best-fit $\rho_{1000}$ values vs. inclination angle for the different SED classes. The size of the plotting symbol increases with the number of objects having the same $\left(i, \rho_{1000}\right)$ combination; the legend in the leftmost panel shows which symbol size corresponds to which number of objects.

Table 4

Median Best-fit Parameter Values for the Three Protostellar Classes

\begin{tabular}{lccc}
\hline \hline Parameter & Class 0 & Class I & Flat-spectrum \\
\hline$L_{\text {tot }}\left(L_{\odot}\right)$ & 5.5 & 2.0 & 3.0 \\
$\rho_{1000}\left(\mathrm{~g} \mathrm{~cm}^{-3}\right)$ & $5.9 \times 10^{-18}$ & $2.4 \times 10^{-19}$ & $1.2 \times 10^{-19}$ \\
$\theta(\mathrm{deg})$ & 25 & 15 & 25 \\
$R_{\text {disk }}(\mathrm{au})$ & 100 & 50 & 5 \\
$i(\mathrm{deg})$ & 70 & 63 & 57 \\
$A_{V}$ & 9.2 & 8.9 & 10.1 \\
\hline
\end{tabular}

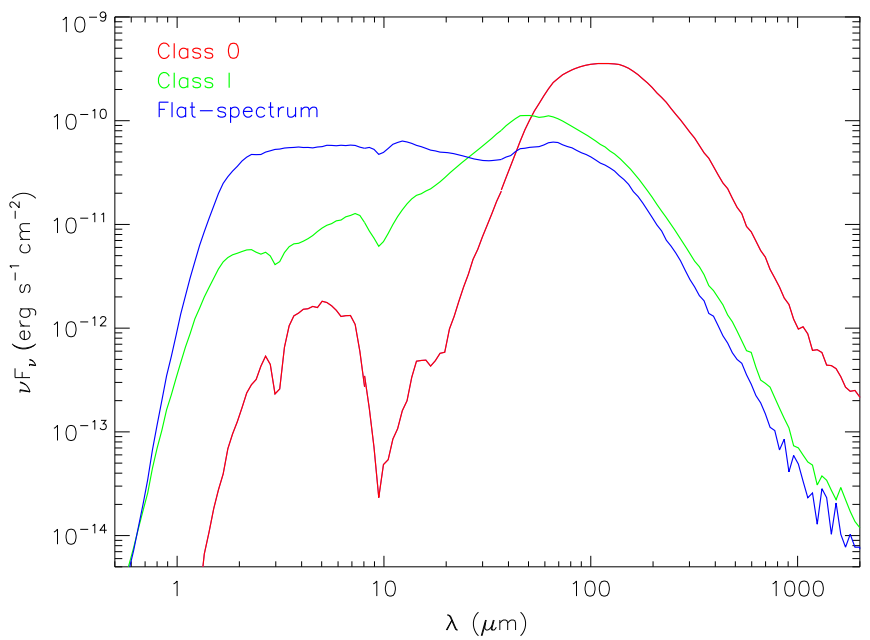

Figure 42. Model SEDs for Class 0 protostars (red), Class I protostars (green), and flat-spectrum sources (blue) with parameter values equal to the median values for each SED class (see Table 4).

geometry. Some SEDs are very flat out to $100 \mu \mathrm{m}$, others have negative spectral slopes beyond $40 \mu \mathrm{m}$, and again others a rising SED from the mid- to the far-IR. There are also objects with more pronounced absorption features due to not only silicates but also ices, as are typically found in Class 0 and I protostars, but also edge-on disks (see HOPS 82, 85, 89, 90, 92, $129,150,200,210,211,281,304,331$, and 363). Only two flat-spectrum sources have prominent silicate emission features, and their SEDs are reminiscent of protoplanetary disks (see HOPS 187 and 199). Thus, flat-spectrum sources likely include objects of a variety of evolutionary stages.
The latter conclusion can also be drawn when analyzing the distribution of envelope reference densities and inclination angles for flat-spectrum sources. In Figure 41, we showed that flat-spectrum sources typically have intermediate inclination angles and lower envelope densities. To compare their properties more directly to Class 0 and I protostars, in Figure 51 we show the median best-fit $\rho_{1000}$ value at each best-fit inclination angle; it is larger for Class 0 and I protostars than for flat-spectrum sources at all inclination angles. For Class 0 and I protostars, the median $\rho_{1000}$ value is highest at intermediate inclination angles, decreases at larger inclination angles, and then increases again for $i>80^{\circ}$. For flat-spectrum sources, the median $\rho_{1000}$ value is relatively flat over the $18^{\circ}-$ $63^{\circ}$ region but has its peak value at $i=41^{\circ}$; it decreases for larger inclination angles. The only flat-spectrum source with a best-fit inclination angle of $81^{\circ}$, HOPS 357 , has a very low envelope density (the lowest value for this parameter in the model grid), and its spectrum displays a deep silicate absorption feature.

Overall, this shows that, while a range of envelope densities and inclination angles can explain flat-spectrum sources, their envelope densities are typically lower than for Class 0 and I protostars. The higher-density objects are seen at low to intermediate inclination angles, while only the lowest-density objects are seen closer to edge-on. Some of the high-density flat-spectrum sources could actually be more embedded protostars (Stage 0 objects) seen face-on (which would be classified as Class 0 objects if seen at larger inclination angles). Thus, in terms of envelope evolution, they include a diverse group of objects.

We note that even though we find that flat-spectrum sources have in general lower envelope densities than Class 0 and Class I objects, their best fit does include an envelope in almost all cases; just 3 of the 102 flat-spectrum sources are best fit without an envelope. This seems to contradict recent findings by Heiderman \& Evans (2015), who found that only about $50 \%$ of flat-spectrum sources were actually protostars surrounded by envelopes. This could be partly explained by different criteria used to select flat-spectrum sources; in the Heiderman \& Evans (2015) sample, flat-spectrum sources are selected by their extinction-corrected 2-24 $\mu \mathrm{m}$ spectral index (see also Evans et al. 2009; Dunham et al. 2013), while our sample uses a flat 


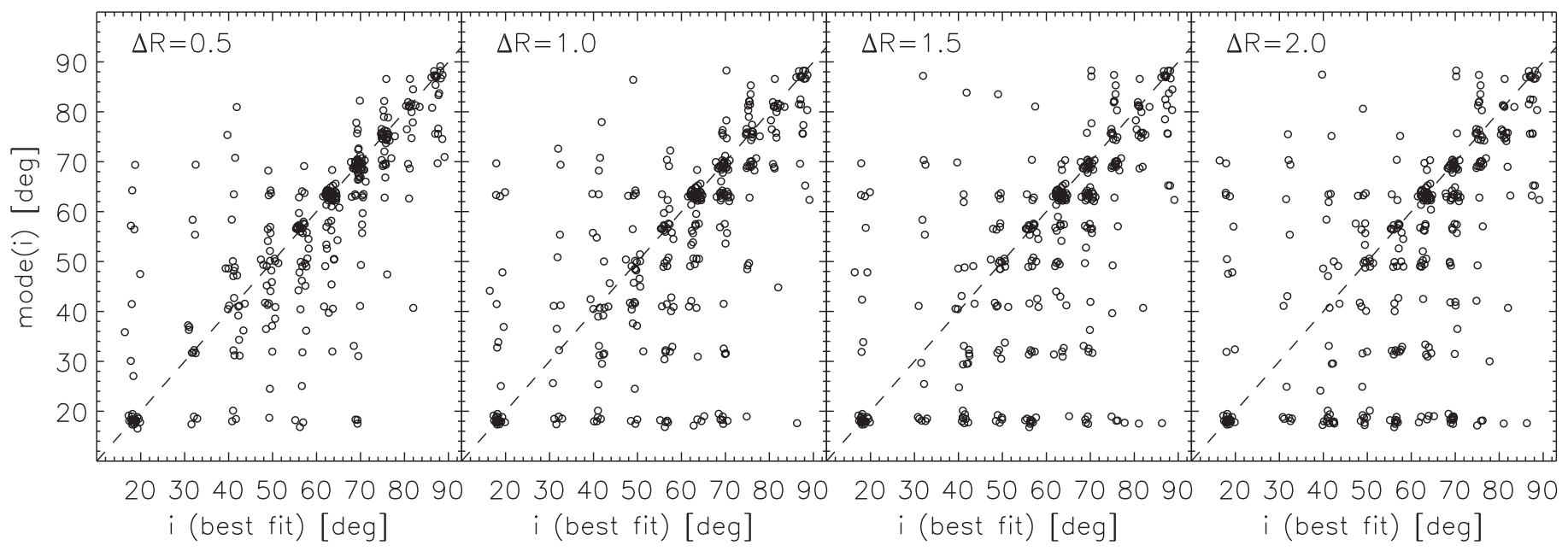

Figure 43. Mode of the inclination angle of all models that lie within $0.5,1.0,1.5$, and 2 of the best-fit $R$ value (from left to right) vs. the best-fit inclination angle for all 330 objects in our sample. Note that for each data point, small random offsets in the $x$ and $y$ direction have been applied to avoid overlap. Also, when two or more parameter values had the same frequency within a $\Delta R$ bin (i.e., not a unique mode value), we computed the average of these values and used it for the mode. The dashed line indicates where the mode and best-fit value are equal.

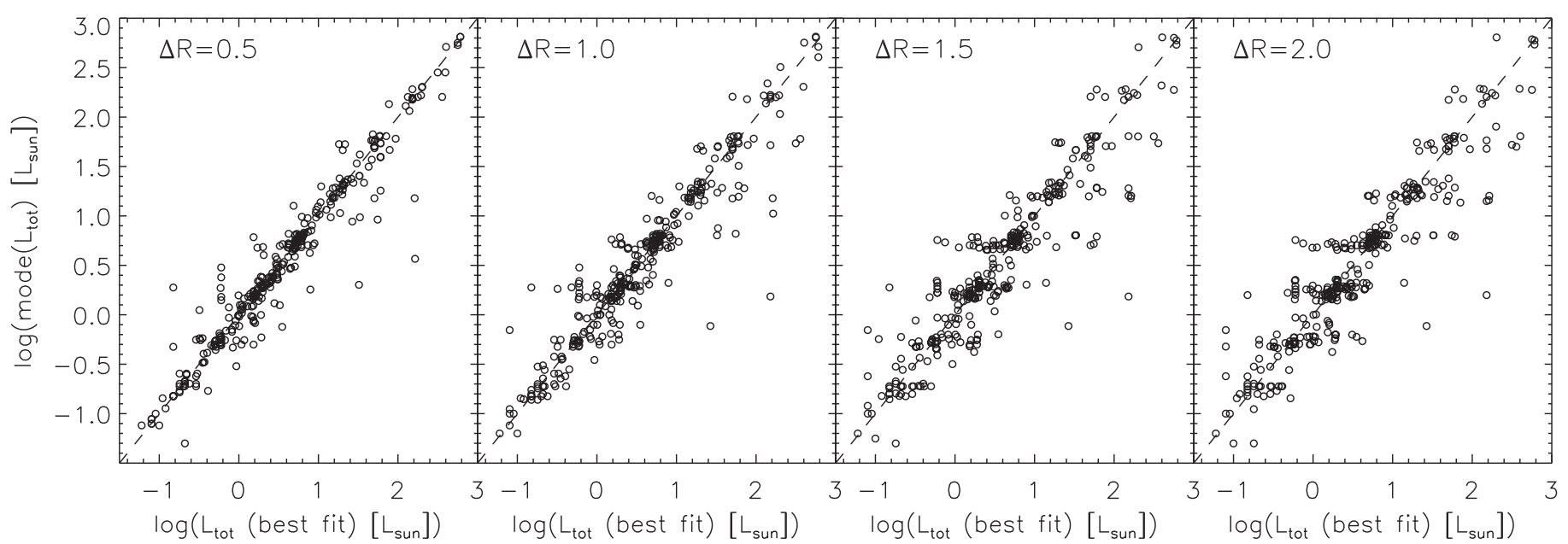

Figure 44. Mode of the total luminosity of all models that lie within $0.5,1.0,1.5$, and 2 of the best-fit $R$ value (from left to right) vs. the best-fit total luminosity for all 330 objects in our sample.

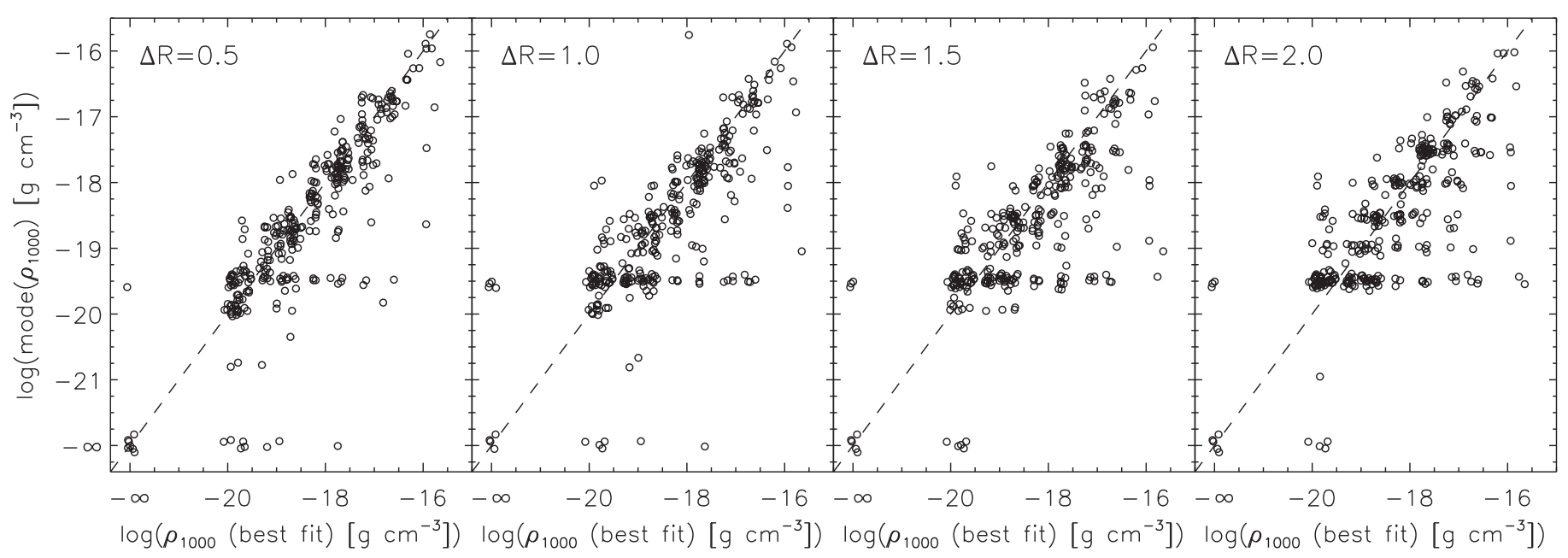

Figure 45. Similar to Figure 43, but for the reference density $\rho_{1000}$. 


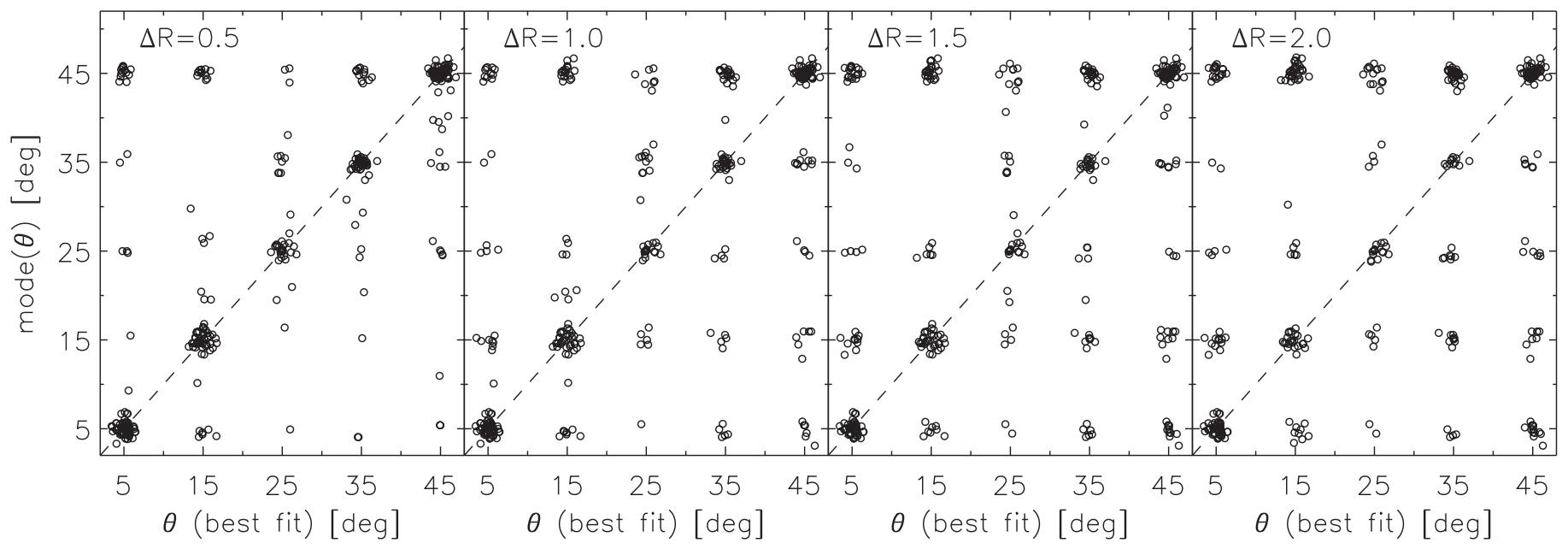

Figure 46. Similar to Figure 43, but for the cavity opening angle.

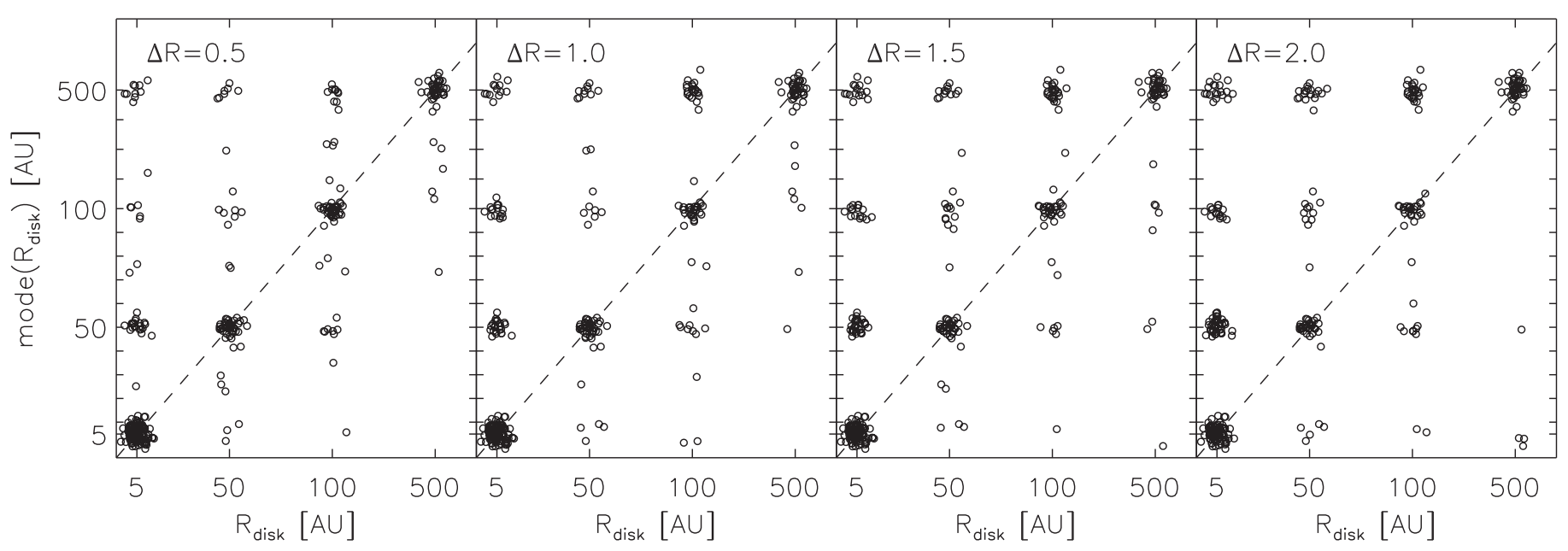

Figure 47. Similar to Figure 43, but for the outer disk radius $\left(=R_{c}\right)$.

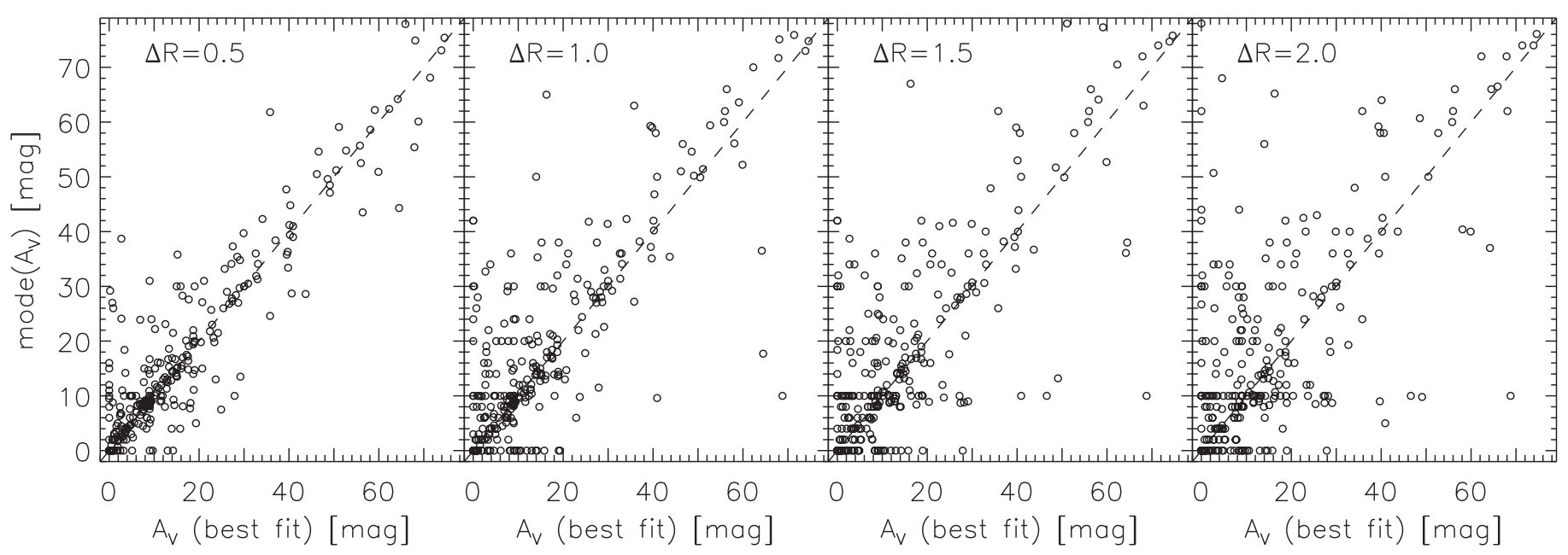

Figure 48. Similar to Figure 44, but for the foreground extinction.

4.5-24 $\mu \mathrm{m}$ spectral index. Moreover, in their study Heiderman \& Evans (2015) detected the presence of an envelope via $\mathrm{HCO}^{+}$emission, and they found that almost all sources detected in the submillimeter are also detected in $\mathrm{HCO}^{+}$(but the opposite does not always hold). For our sample of Orion protostars, we find that $75 \%$ of Class $0+\mathrm{I}$ protostars observed with SABOCA $(350 \mu \mathrm{m})$ are detected, while only $47 \%$ of flatspectrum sources have detections. For LABOCA observations 


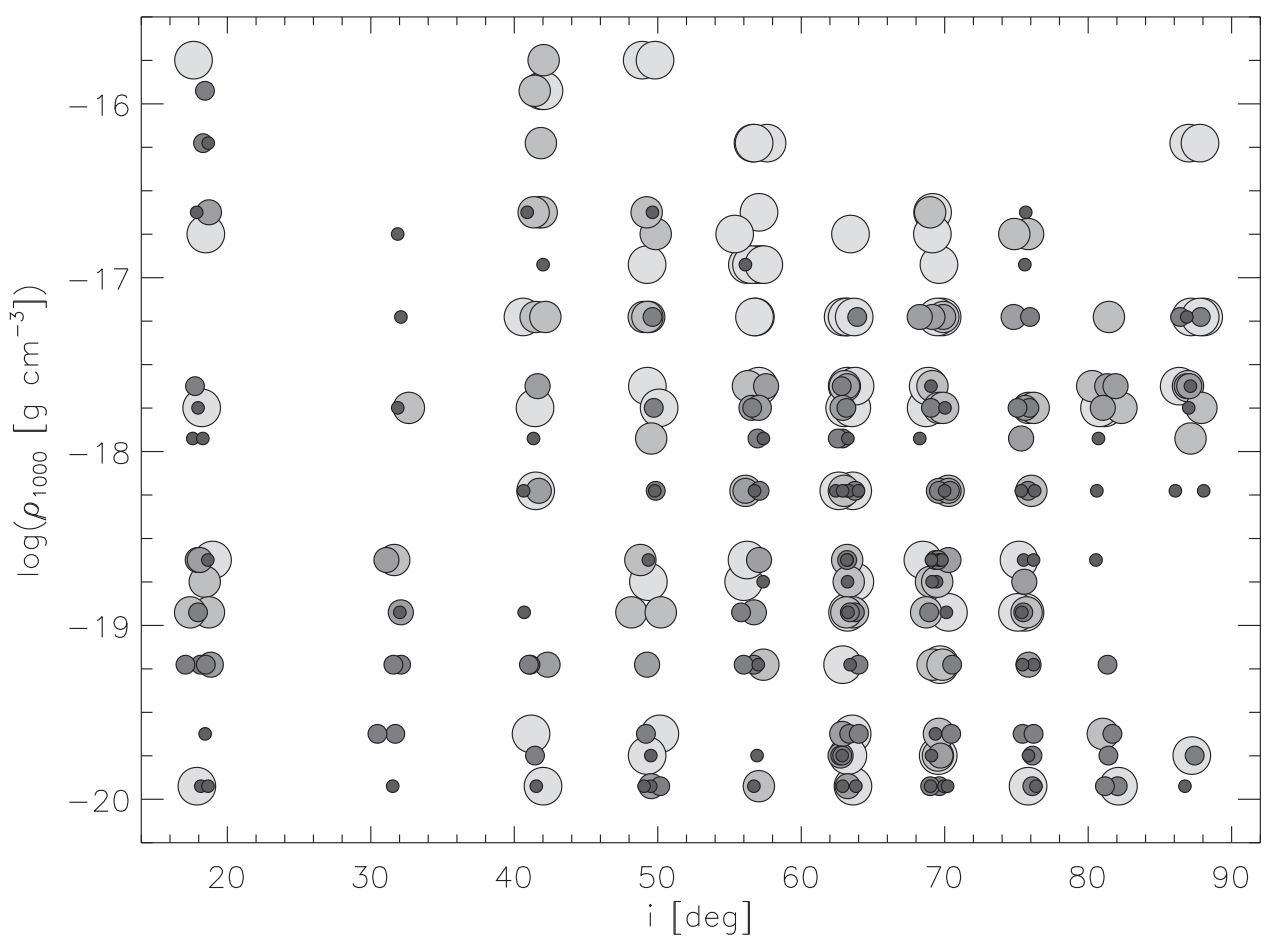

Figure 49. Best-fit $\rho_{1000}$ values vs. inclination angle with the cavity size indicated by the different symbol sizes and gray shades: symbols become larger and lighter colored with increasing cavity size $\left(5^{\circ}, 15^{\circ}, 25^{\circ}, 35^{\circ}, 45^{\circ}\right)$. A small random offset in the $x$ direction has been applied to each data point to prevent too much overlap.

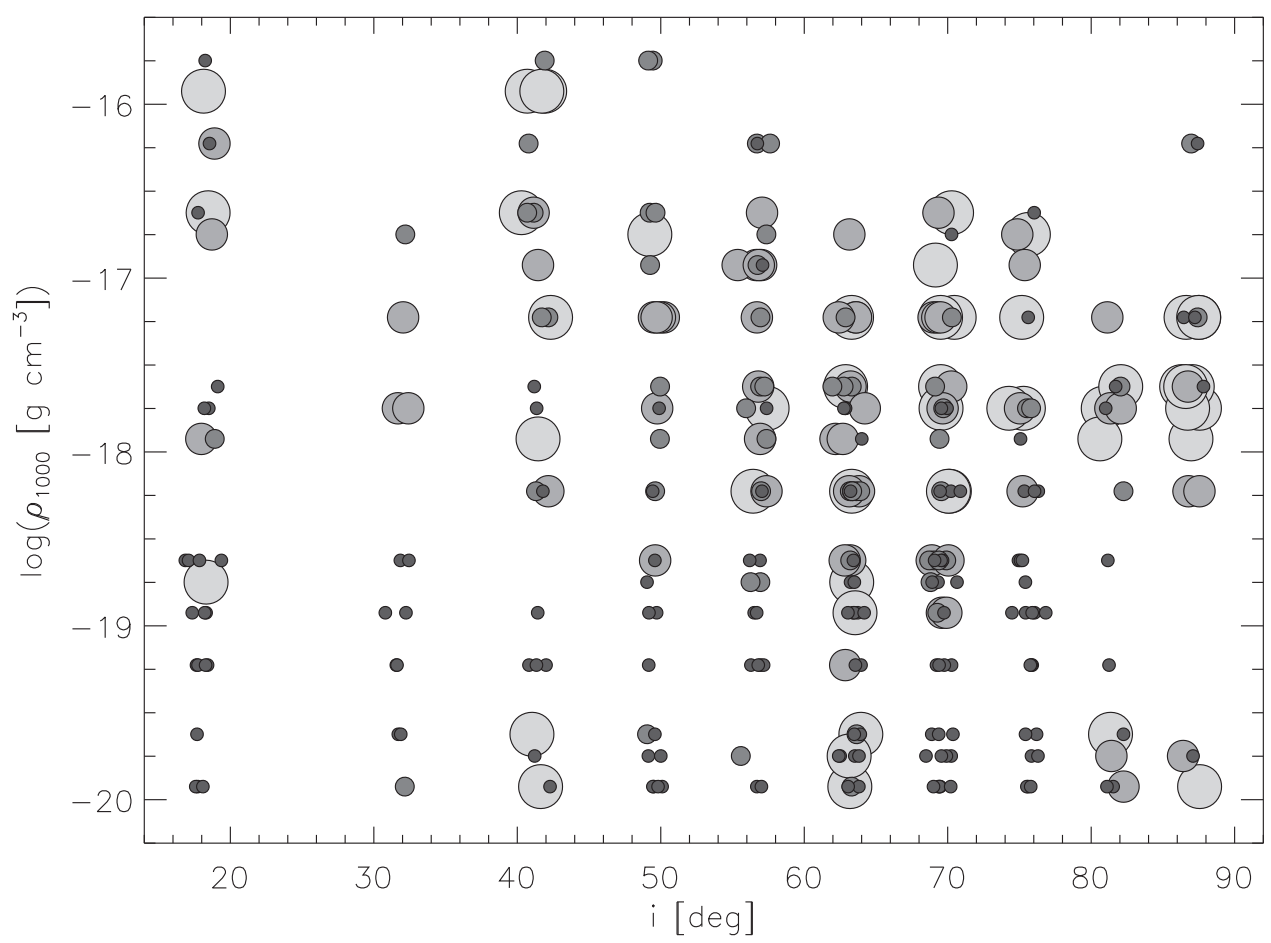

Figure 50. Similar to Figure 49, but with the outer disk radius indicated by the different symbol sizes and gray shades: symbols become larger and lighter colored with increasing disk radius $(5,50,100,500 \mathrm{au})$.

$(870 \mu \mathrm{m})$, these two fractions amount to $41 \%$ and $21 \%$, respectively. Thus, we find that flat-spectrum sources have a $\sim 50 \%$ lower submillimeter detection rate than Class $0+\mathrm{I}$ protostars. Flat-spectrum sources without submillimeter detections would likely also not display $\mathrm{HCO}^{+}$emission and thus would be considered as protostars without envelopes by Heiderman \& Evans (2015).

To compare how our submillimeter detections correlate with the presence of an envelope, in Figures 52 and 53 we show the derived best-fit reference envelope densities as a function of 


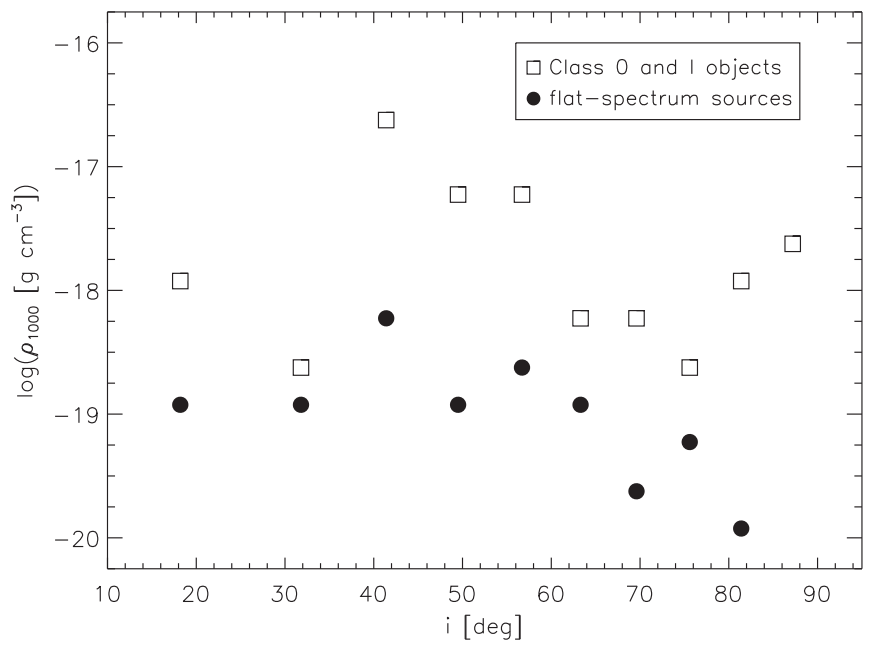

Figure 51. Median best-fit $\rho_{1000}$ values at each inclination angle for the Class 0 and I protostars (squares) and the flat-spectrum sources (circles) in our sample.

350 or $870 \mu \mathrm{m}$ fluxes for the combined Class $0+\mathrm{I}$ sample and the flat-spectrum sources. We also differentiate the distribution of envelope densities between measured flux values and upper limits; at $870 \mu \mathrm{m}$, the upper limits are often cases where the sources are not detected due to confusion with bright, spatially varying emission. We find that even protostars with upper limits at 350 and $870 \mu \mathrm{m}$ are best fit with an envelope; however, the envelope density is lower for objects with upper limits in the submillimeter. This is especially evident for Class $0+\mathrm{I}$ protostars; for flat-spectrum sources, the distributions of envelope densities for submillimeter detections and upper limits show significant overlap. Four times as many flatspectrum sources have upper limits instead of detections at $870 \mu \mathrm{m}$, but their derived $\rho_{1000}$ values span almost the full range of values. Furthermore, the median $\rho_{1000}$ value of $1.19 \times 10^{-19} \mathrm{~g} \mathrm{~cm}^{-3}$ for sources without detections is relatively close to the median value of $5.95 \times 10^{-19} \mathrm{~g} \mathrm{~cm}^{-3}$ for the sources with $870 \mu \mathrm{m}$ detections. Thus, our model fits do not rely on submillimeter detections to yield a best fit with an envelope; in most cases the near- to far-IR SED is sufficient to constrain the properties of the envelope.

\subsubsection{Sources without an Envelope and Class II Objects}

Among the six objects whose best-fit SED required no envelope $\left(\rho_{1000}\right.$ value of 0$)$, three are flat-spectrum sources (HOPS 47, 187, 265), two are Class II pre-main-sequence stars (HOPS 113, 293), and one is a Class I protostar (HOPS 232). The low $70 \mu \mathrm{m}$ fluxes of HOPS 47 and 265 constrained the best model to one without an envelope. The SED of HOPS 187 looks like that of a transitional disk, which are disks with gaps or holes in their inner regions (see Espaillat et al. 2014 and references therein). If HOPS 187 were a transitional disk, it would not have an envelope. HOPS 232 has a rising SED over the mid-IR spectral range; its best fit requires no envelope, but an edge-on disk with a high accretion luminosity.

It would be expected that the SEDs of Class II objects can be best fit by a model that does not include an envelope. This is the case for HOPS 113 and 293. Of the nine remaining Class II objects in our sample, four have very low envelope densities $\left(\rho_{1000} \sim(1-2.5) \times 10^{-20} \mathrm{~g} \mathrm{~cm}^{-3}\right.$; HOPS 22, 26, 98, 283), while five have $\rho_{1000}$ between $6.0 \times 10^{-20}$ and $1.8 \times 10^{-19} \mathrm{~g} \mathrm{~cm}^{-3}$ (HOPS 184, 201, 222, 272, 277). The
SEDs of HOPS 22, 184, and 201 are similar to those of transitional disks, with some silicate emission at $10 \mu \mathrm{m}$ and a rising SED between about 13 and $20 \mu \mathrm{m}$. The best-fit models require some envelope emission to fit the long-wavelength data. HOPS 222, 272, and 277 lie close to the border between a Class II pre-main-sequence star and a flat-spectrum source based on their 4.5-24 $\mu \mathrm{m}$ spectral index, and therefore they could have some envelope material left, despite being classified as Class II objects.

Overall, of the 330 YSOs in our sample, 319 were classified as either Class 0, Class I, or flat-spectrum protostars based on their SEDs. However, 4 of them are best fit without an envelope. Conversely, of the 11 Class II objects in our sample, 9 are best fit with an envelope; however, three of these might be transitional disks. Thus, based on our model fits and SEDs, 321 of our 330 YSOs are protostars with envelopes, and 9 are likely pre-main-sequence stars with disks.

\subsection{The Total Luminosities of Protostars}

The luminosity distribution of protostars is a significant constraint on protostellar evolution, and it is important to understand the effect of the envelope on the observed luminosity (e.g., Offner \& McKee 2011). The bolometric luminosity distribution of the HOPS protostars is very similar to that determined for the Spitzer-identified protostars by Kryukova et al. (2012) with a peak near $1 L_{\odot}$ (Figure 2 ). In contrast, the distribution of the total luminosities from the models shows a peak near $2.5 L_{\odot}$ (Figure 36 ), indicating that the luminosities of protostars may be systematically underestimated by the bolometric luminosities, which do not take into account the inclination angle (and thus beaming of the radiation along the outflow cavities), as well as foreground extinction (see Figure 32 in Section 6.2).

Higher intrinsic luminosities for protostars could help address the "luminosity problem" first pointed out by Kenyon et al. (1990), who found that the luminosities of protostars are lower by about an order of magnitude than a simple estimate of the expected accretion luminosity. However, an increase in the luminosity by a factor of 2.5-3 would not solve the problem; solutions proposed by other authors, such as mass-dependent accretion rates (Offner \& McKee 2011) or episodic accretion events (Dunham \& Vorobyov 2012), are still needed.

Our best-fit models also suggest that Class 0 protostars have a different distribution of $L_{\text {tot }}$ values compared to Class I protostars or flat-spectrum sources. Their median total luminosity is higher, which could be an indication of larger accretion luminosities for younger protostars. We must bear in mind the caveats and degeneracies mentioned above; in particular, in some cases the higher luminosity could be related to the adoption of an overly large inclination angle, which results in most of the emitted radiation not reaching the observer. Nevertheless, these differences have potentially important implications for protostellar evolution, which will be discussed in a future publication (W. Fischer et al. 2016, in preparation).

\subsection{Potential Problems with TSC Models}

Although the TSC models provide impressive fits to the SEDs, some of the observed trends suggest problems with the models. First, the distribution of inclination angles (Figure 29) deviates from what we expect from a randomly oriented sample 

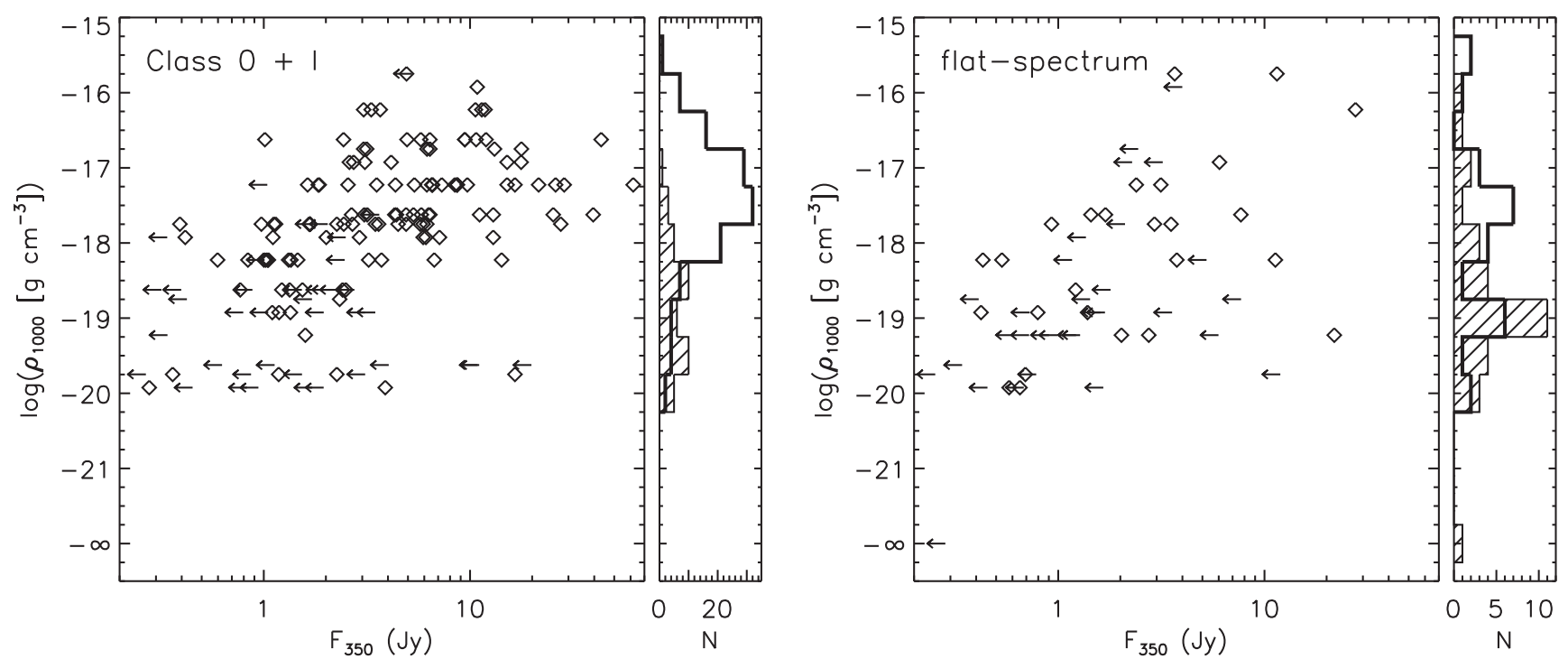

Figure 52. Best-fit $\rho_{1000}$ values vs. the $350 \mu \mathrm{m}$ fluxes for the Class 0 and I protostars (left) and the flat-spectrum sources (right) in our sample. Detections at $350 \mu \mathrm{m}$ are shown with diamonds, while upper limits are shown with arrows. The histograms show the distribution of best-fit $\rho_{1000}$ values for sources with a $350 \mu \mathrm{m}$ flux measurement (thick solid line) and with $350 \mu \mathrm{m}$ upper limits (shaded area).
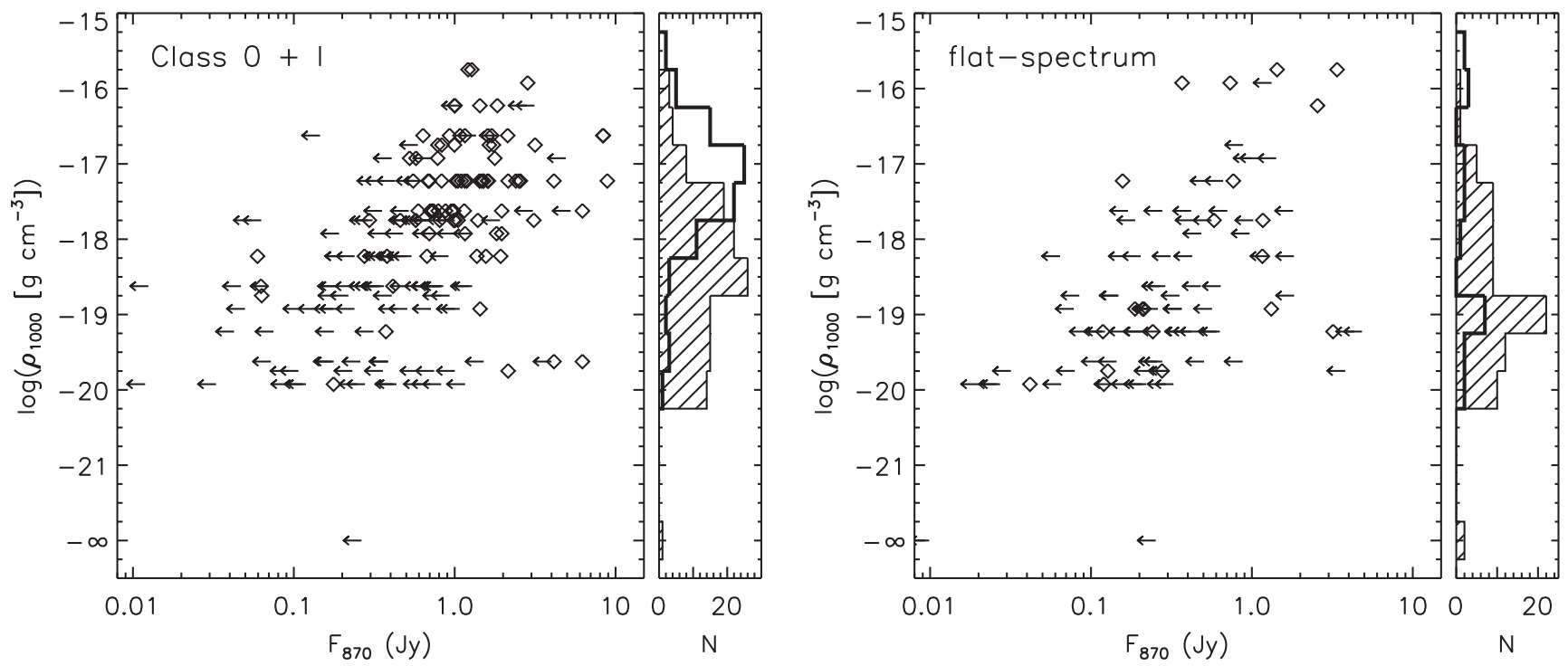

Figure 53. Similar to Figure 52, but for the $870 \mu \mathrm{m}$ fluxes.

of protostars. Although this could result from unintentional selection biases in our sample of protostars, it may also be the effect of applying the wrong envelope model to the data.

Furthermore, our data show flat distributions in cavity open angles for Class 0 and flat-spectrum sources, but an excess of small cavities for the Class I protostars (Figure 35). We also find that protostars with large cavities often have high envelope densities (Figure 49). For example, models with high envelope densities viewed more edge-on require large cavity opening angles and high $L_{\text {tot }}$ values to generate sufficient mid-IR flux; this is the case for a few of our highest-luminosity objects (HOPS 87, 108, and 178). These trends do not support the notion of increasing cavity size with later evolutionary stage, which would be expected if outflows play a major role in dispersing envelopes (Arce \& Sargent 2006). This may suggest that cavity sizes are not growing with time; however, this may also imply a deviation from spherical symmetry for the initial configuration of the collapsing envelopes. Such a deviation may result if the envelope collapses from the fragmentation of a flattened sheet or elongated filament.

Finally, we find an excess of small values of $R_{\text {disk }}$, and therefore small centrifugal radii, for Class I and flat-spectrum protostars (Figure 37). This is contrary to the expectation from the TSC model, in which the late stages of protostellar evolution are characterized by the infall of high angular momentum material from large radii and hence larger values of $R_{c}$. This may imply that disks sizes are small, but it may also be the result of incorrect assumptions about the distribution of angular momentum in the TSC model.

In total, these "conundrums" that arise from our model fits hint that the current models do not realistically reproduce the structure of collapsing envelopes. Future high-resolution observations at submillimeter and longer wavelengths that resolve the structure and motions of envelopes may provide the means to develop more refined models that can fit the SEDs with more realistic envelope configurations. 


\section{CONCLUSIONS}

We have presented SEDs and model fits for 330 YSOs in the Orion A and B molecular clouds. The SEDs include data from 1.2 to $870 \mu \mathrm{m}$, with near-infrared photometry from 2MASS, mid-infrared photometry and spectra from the Spitzer Space Telescope, far-infrared photometry at 70, 100, and $160 \mu \mathrm{m}$ from the Herschel Space Observatory, and submillimeter photometry from the APEX telescope. We calculated bolometric luminosities $\left(L_{\text {bol }}\right)$, bolometric temperatures $\left(T_{\text {bol }}\right)$, and $4.5-24 \mu \mathrm{m}$ spectral indices $\left(n_{4.5-24}\right)$ for all 330 sources in our sample. From the distributions of these three parameters, we find that $L_{\mathrm{bol}}$ has a broad peak near $1 L_{\odot}$ and extends from 0.02 to several hundred $L_{\odot}$, while the distribution of $T_{\text {bol }}$ values is broad and flat from about 30 to $800 \mathrm{~K}$, with a median value of $146 \mathrm{~K}$. The $4.5-24 \mu \mathrm{m}$ spectral indices range from -0.75 to 2.6, with a peak near 0 .

Based on traditional classification schemes involving $n_{4.5-24}$ and $T_{\text {bol }}$, we have identified 92 sources as Class 0 protostars $\left(n_{4.5-24}>0.3\right.$ and $\left.T_{\text {bol }}<70 \mathrm{~K}\right), 125$ as Class I protostars $\left(n_{4.5-24}>0.3\right.$ and $\left.T_{\text {bol }}>70 \mathrm{~K}\right)$, and 102 as flat-spectrum sources $\left(-0.3<n_{4.5-24}<0.3\right)$. The remaining 11 sources are Class II pre-main-sequence stars with $n_{4.5-24}<-0.3$; most of them just missed the flat-spectrum cutoff, and three have SEDs typical of disks with inner holes. Considering these transitional disks and YSOs whose best fit does not require an envelope, we find that 321 of the 330 HOPS targets in our sample are protostars with envelopes. Class 0 and I protostars often display a deep silicate absorption feature at $10 \mu \mathrm{m}$ owing to the presence of the envelope, while many flat-spectrum sources have a weak silicate emission or absorption feature at that wavelength.

We have used a grid of 30,400 protostellar model SEDs, calculated using the 2008 version of the Whitney et al. (2003b, 2003a) Monte Carlo radiative transfer code, to find the best-fit models for each observed SED. The grid is limited to discrete values for protostellar parameters, and their ranges were chosen to represent typical protostars. Within the framework of these models, we find the following:

1. About $70 \%$ of Class 0 protostars, $75 \%$ of Class I protostars, and close to $90 \%$ of flat-spectrum sources have reliable SED fits $(R<4$, where $R$ is a measure of the average distance between model and data in units of the fractional uncertainty). Thus, our model grid can reproduce most of the observed SEDs of Orion protostars.

2. Our results show a clear trend of decreasing envelope densities as we progress from Class 0 to Class I and then to flat-spectrum sources: we find that the median $\rho_{1000}$ values decrease from $5.9 \times 10^{-18} \mathrm{~g} \mathrm{~cm}^{-3}$ to $2.4 \times 10^{-19} \mathrm{~g} \mathrm{~cm}^{-3}$ to $1.2 \times 10^{-19} \mathrm{~g} \mathrm{~cm}^{-3}$. The decrease in densities implies a decrease in the infall rates of the protostars as they evolve. We find that the PBRs have median $\rho_{1000}$ values twice as high as the median value of the Class 0 protostars in our sample, supporting the interpretation that they are likely the youngest protostars in Orion.

3. There are degeneracies in the parameters for models that reproduce the observed SEDs. For example, increasing the mid-IR SED slope and deepening the silicate absorption feature at $10 \mu \mathrm{m}$ of a model protostar can be done by increasing the envelope density or inclination angle, decreasing the cavity opening angle or centrifugal radius, or even increasing the foreground extinction. Hence, the properties of a specific source may be fit by a wide range of parameters. The best-fit model parameters are particularly uncertain for objects whose SED is not well-constrained by observations. Because of these degeneracies, the observed classes contain a mixture of evolutionary stages.

4. We find that flat-spectrum sources are particularly well fit by our models. They have, on average, lower envelope densities and intermediate inclination angles, so many flat-spectrum sources are likely more evolved protostars, but this group also includes protostars with higher envelope densities (and sometimes larger cavity opening angles) seen at lower inclination angles. Flat-spectrum sources seen at $i>65^{\circ}$ have very tenuous envelopes. Thus, the sample of flat-spectrum sources includes protostars at different stages in their envelope evolution. All but three of the flat-spectrum sources in our sample have envelopes in their best-fit models, indicating that, with a small number of exceptions, these objects are protostars with infalling gas.

5. The luminosity function for the model luminosities peaks at a higher luminosity than that for the observed bolometric luminosities as a result of beaming along the outflow cavities. Furthermore, the total luminosity determined by the models is higher for Class 0 protostars: the median total luminosities are 5.5, 2.0, and $3.0 L_{\odot}$ for Class 0, Class I, and flat-spectrum sources, respectively.

6. Since heating by external radiation fields is not included in our model grid, we assessed its influence by adding an ISRF to a set of models. We find that an ISRF 10 times that typical of the solar neighborhood can substantially change the SEDs of sources with internal luminosities of $0.1 L_{\odot}$. However, when we incorporate the effect of extinction on the external radiation field, the effect on the protostellar SEDs is smaller; the best-fit luminosities and envelope densities would be overestimated by factors of a few for $\sim 0.1 L_{\odot}$ prototars and much less for higherluminosity protostars. We estimate that the best-fit parameters (in particular, $L_{\mathrm{tot}}, \rho_{1000}$ ) of $\sim 20 \%$ of the HOPS sources could be affected by external heating.

7. Although the adopted TSC models reproduce the observed SEDs well, there are trends that suggest inadequacies with these models. First, the distribution of best-fit inclination angles does not reproduce that expected for randomly oriented protostars. Second, although the distribution of outflow cavity sizes for flatspectrum and Class 0 sources is flat, there is an excess of small cavities for Class I sources. This is in contradiction to the typical picture that outflow cavities grow as protostars evolve. Finally, the distribution of outer disk radii set by the rotation of the envelope is concentrated at small values $(<50 \mathrm{au})$ for the Class I and flat-spectrum sources but is slightly tilted toward large values $(>50 \mathrm{au})$ for Class 0 protostars. Again, this trend contradicts the expected growth of disks as the infall region in protostellar envelopes expands. These findings suggest that either the envelope structure of the adopted models is incorrect or our understanding of the evolution of protostars needs to be revised substantially.

Our work provides a large sample of protostars in one molecular cloud complex for future, more detailed studies of protostellar evolution. For example, using additional constraints, such as from scattered light imaging, the structure of 
envelope cavities and thus the role of outflows can be better understood. In addition, the detailed structure of the envelope and the disk embedded within, as well as multiplicity of the central source, can be studied with high spatial resolution imaging such as ALMA can provide. With the analysis of their SEDs presented in this work, the HOPS protostars constitute an ideal sample to derive a better understanding of the early evolution of young stars, when the assembly of the stellar mass and the initial stages of planet formation likely take place.

Support for this work was provided by NASA through awards issued by JPL/Caltech. The work of W.J.F. was supported in part by an appointment to the NASA Postdoctoral Program at Goddard Space Flight Center, administered by Oak Ridge Associated Universities through a contract with NASA. J.J.T. acknowledges support provided by NASA through Hubble Fellowship grant \#HST-HF-51300.01-A awarded by the Space Telescope Science Institute, which is operated by the Association of Universities for Research in Astronomy, Inc., for NASA, under contract NAS 5-26555. J.J.T. acknowledges further support from grant 639.041.439 from the Netherlands Organisation for Scientific Research (NWO). The work of A. M.S. was supported by the Deutsche Forschungsgemeinschaft priority program 1573 ("Physics of the Interstellar Medium"). M.O. acknowledges support from MINECO (Spain) AYA2011-3O228-CO3-01 and AYA2014-57369-C3-3-P grants (co-funded with FEDER funds). We thank Thomas Robitaille for helpful discussions regarding the model grid and model parameters. This work is based on observations made with the Spitzer Space Telescope, which is operated by the Jet Propulsion Laboratory (JPL), California Institute of Technology (Caltech), under a contract with NASA; it is also based on observations made with the Herschel Space Observatory, a European Space Agency Cornerstone Mission with significant participation by NASA. The Herschel spacecraft was designed, built, tested, and launched under a contract to ESA managed by the Herschel/Planck Project team by an industrial consortium under the overall responsibility of the prime contractor Thales Alenia Space (Cannes), and including Astrium (Friedrichshafen) responsible for the payload module and for system testing at spacecraft level, Thales Alenia Space (Turin) responsible for the service module, and Astrium (Toulouse) responsible for the telescope, with in excess of 100 subcontractors. We also include data from the Atacama Pathfinder Experiment, a collaboration between the Max-Planck Institut für Radioastronomie, the European Southern Observatory, and the Onsala Space Observatory. This publication makes use of data products from the Two Micron All Sky Survey, which is a joint project of the University of Massachusetts and the Infrared Processing and Analysis Center/Caltech, funded by NASA and the NSF.

\section{APPENDIX A \\ SPECTRAL ENERGY DISTRIBUTIONS AND VARIABILITY}

The data sets used for the SEDs presented in this work were taken with different instruments and telescopes and are not contemporaneous, yet we know that the majority of protostars are variable at a $\sim 20 \%$ level (e.g., Morales-Calderón et al. 2011; Billot et al. 2012; Megeath et al. 2012). Therefore, different data sets are snapshots of the emission of the protostar at particular times of its unknown duty cycle of variability. Indeed, in some cases we observe large mismatches between different data sets; an extreme example, the outbursting protostar HOPS 223, was studied by Fischer et al. (2012). Another HOPS protostar that recently experienced an outburst, HOPS 383 (Safron et al. 2015), does not have a mismatched SED, since the photometry used here is representative of the post-outburst SED. In general, variability that is wavelength dependent or has a long duty cycle is more difficult to determine.

Since 290 of the 330 objects in the HOPS sample that were modeled have an IRS spectrum and measurements with IRAC or MIPS, we compared fluxes measured in the same wave bands, but at different times, to see whether there are discrepancies. We used the Spitzer Science Center's spitzer_synthphot code to calculate IRAC 5.8 and $8.0 \mu \mathrm{m}$ and MIPS $24 \mu \mathrm{m}$ synthetic photometry from the IRS fluxes. In Figure 54, we show the flux ratios of IRAC or MIPS photometry and the synthetic photometry using the IRS spectrum for the protostars in our HOPS sample. If there were no mismatches, the flux ratios of IRAC or MIPS and IRS photometry would be close to 1 . However, we find that they are typically somewhat less than 1 ; the median ratios at 5.8 ,

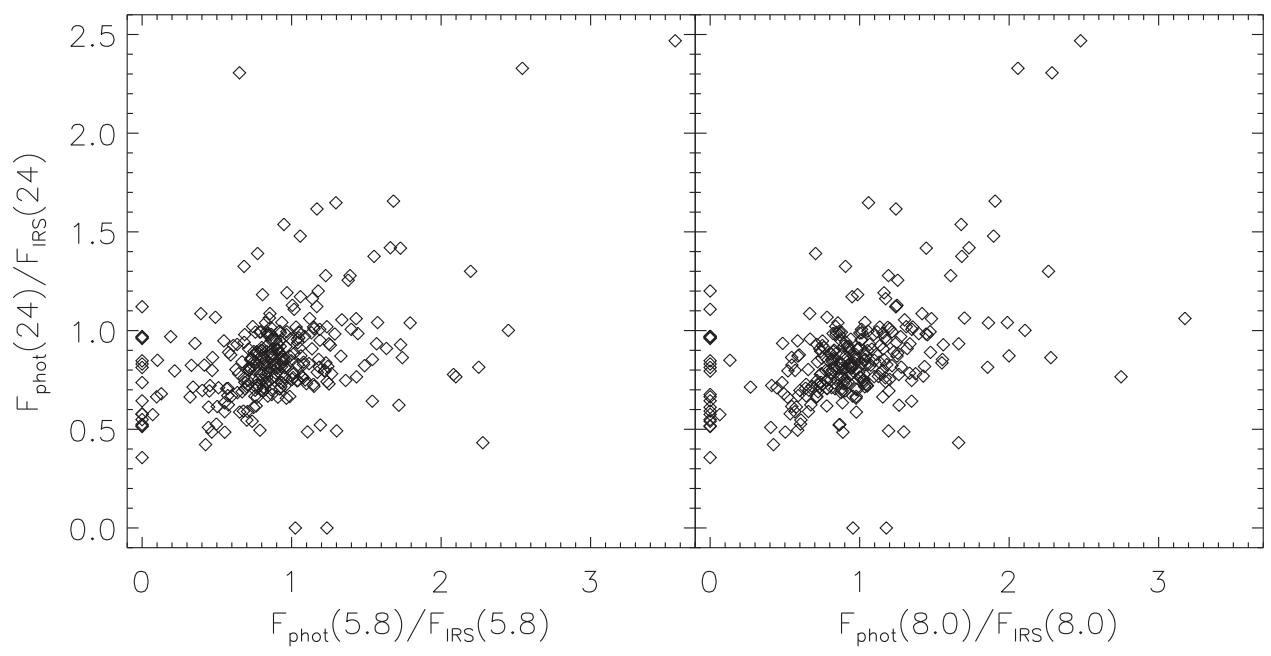

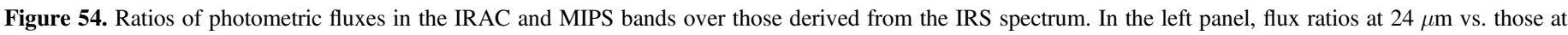

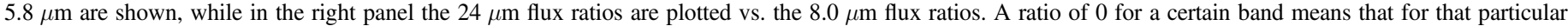
object, the IRS spectrum was not available over the wavelength region of that band. 
Table 5

Potentially Variable HOPS Targets

\begin{tabular}{|c|c|c|c|c|}
\hline $\begin{array}{l}\text { Object } \\
\text { (1) }\end{array}$ & $\begin{array}{l}\text { Class } \\
\text { (2) }\end{array}$ & $\begin{array}{l}\text { [5.8] Ratio } \\
\text { (3) }\end{array}$ & $\begin{array}{l}\text { [8.0] Ratio } \\
\text { (4) }\end{array}$ & $\begin{array}{c}\text { [24] Ratio } \\
\text { (5) }\end{array}$ \\
\hline HOPS 3 & flat & 1.681 & 1.906 & 1.656 \\
\hline HOPS 7 & 0 & 1.121 & 3.176 & 1.061 \\
\hline HOPS 11 & 0 & 0.444 & 0.405 & 0.510 \\
\hline HOPS 12 & 0 & 0.553 & 0.888 & 0.485 \\
\hline HOPS 19 & flat & 1.296 & 1.059 & 1.648 \\
\hline HOPS 20 & I & 2.543 & 2.059 & 2.329 \\
\hline HOPS 24 & I & 0.329 & 0.953 & 0.824 \\
\hline HOPS 32 & 0 & 1.575 & 1.988 & 1.041 \\
\hline HOPS 38 & 0 & 3.566 & 2.478 & 2.468 \\
\hline HOPS 41 & I & 0.788 & 0.586 & 0.495 \\
\hline HOPS 65 & I & 0.191 & 1.275 & 0.969 \\
\hline HOPS 71 & I & 0.416 & 0.547 & 0.825 \\
\hline HOPS 78 & 0 & 0.097 & $\ldots$ & 0.664 \\
\hline HOPS 85 & flat & 1.728 & 1.445 & 1.418 \\
\hline HOPS 91 & 0 & 0.511 & 0.446 & 0.711 \\
\hline HOPS 95 & 0 & 2.450 & 2.106 & 1.001 \\
\hline HOPS 108 & 0 & $\ldots$ & 0.867 & 0.525 \\
\hline HOPS 114 & I & 0.102 & 0.132 & 0.850 \\
\hline HOPS 121 & 0 & $\cdots$ & $\cdots$ & 0.357 \\
\hline HOPS 124 & 0 & 0.402 & 0.601 & 0.697 \\
\hline HOPS 131 & I & 0.341 & 0.270 & 0.714 \\
\hline HOPS 132 & flat & 1.717 & 1.264 & 0.622 \\
\hline HOPS 138 & 0 & 0.354 & 0.485 & 0.935 \\
\hline HOPS 141 & flat & 0.393 & 1.418 & 1.086 \\
\hline HOPS 143 & I & 1.793 & 1.862 & 1.039 \\
\hline HOPS 154 & I & 2.252 & 1.856 & 0.814 \\
\hline HOPS 177 & I & 1.740 & 2.279 & 0.863 \\
\hline HOPS 181 & I & 1.301 & 1.194 & 0.492 \\
\hline HOPS 182 & 0 & 0.772 & 0.705 & 1.390 \\
\hline HOPS 183 & flat & 0.466 & 0.502 & 0.486 \\
\hline HOPS 186 & I & 1.062 & 1.999 & 0.872 \\
\hline HOPS 187 & flat & 0.321 & 1.155 & 0.664 \\
\hline HOPS 203 & 0 & $\ldots$ & 0.481 & 0.737 \\
\hline HOPS 206 & 0 & 1.106 & 1.295 & 0.487 \\
\hline HOPS 222 & II & 0.496 & 0.604 & 0.526 \\
\hline HOPS 223 & I & 0.070 & 0.065 & 0.575 \\
\hline HOPS 228 & I & 2.098 & 2.749 & 0.766 \\
\hline HOPS 239 & I & 0.682 & 0.905 & 1.325 \\
\hline HOPS 270 & I & 1.729 & 1.560 & 0.928 \\
\hline HOPS 271 & I & 0.948 & 1.679 & 1.538 \\
\hline HOPS 272 & II & 1.660 & 1.732 & 1.420 \\
\hline HOPS 278 & I & 0.651 & 2.287 & 2.306 \\
\hline HOPS 290 & 0 & 2.198 & 2.263 & 1.301 \\
\hline HOPS 297 & I & 2.083 & 1.263 & 0.777 \\
\hline HOPS 299 & I & 0.454 & 0.412 & 0.722 \\
\hline HOPS 305 & flat & 0.424 & 0.423 & 0.422 \\
\hline HOPS 316 & 0 & 2.279 & 1.662 & 0.432 \\
\hline HOPS 319 & I & 0.218 & 0.520 & 0.796 \\
\hline HOPS 321 & I & 0.769 & 0.518 & 0.662 \\
\hline HOPS 322 & I & 0.128 & 0.494 & 0.680 \\
\hline HOPS 338 & 0 & 1.552 & 1.683 & 1.376 \\
\hline HOPS 340 & 0 & 1.192 & 0.863 & 0.522 \\
\hline HOPS 358 & 0 & $\ldots$ & $\cdots$ & 0.514 \\
\hline HOPS 359 & 0 & $\cdots$ & $\cdots$ & 0.517 \\
\hline HOPS 363 & flat & 1.169 & 1.242 & 1.616 \\
\hline HOPS 388 & flat & 1.058 & 1.896 & 1.479 \\
\hline
\end{tabular}

Note. Column (1) lists the HOPS number of the object, column (2) the class based on SED classification, column (3) the ratio of the IRAC $5.8 \mu \mathrm{m}$ flux and the IRS flux over the IRAC $5.8 \mu \mathrm{m}$ band, columns (4) and (5) the ratio of photometric and IRS flux for the IRAC $8.0 \mu \mathrm{m}$ and MIPS $24 \mu \mathrm{m}$ band, respectively.
8.0 , and $24 \mu \mathrm{m}$ are $0.89,0.95$, and 0.83 , respectively. For small mismatches, calibration uncertainties are a plausible explanation. In addition, since we are dealing with objects that are not necessarily point sources and often embedded in extended emission, differences in aperture sizes between different measurements (IRAC versus MIPS versus IRS) could also account for flux mismatches.

To identify outliers, in Table 5, we list those flux ratios that lie in the lower or upper 5\% of values. They represent a conservative list of potentially variable sources in our sample. Of the 290 objects for which we calculated flux ratios, 5 have flux mismatches larger than a factor of 2 between IRS and both IRAC bands at 5.8 and $8.0 \mu \mathrm{m}$. Three objects have similarly large mismatches between IRS and MIPS. The overlap between these two samples contains two objects, HOPS 20 and 38 (the other objects are HOPS 95, 228, 278, and 290). For most of these objects, the large mismatches can be attributed to noisy IRS spectra, especially in the 5-8 $\mu \mathrm{m}$ region, making the comparison between IRAC and IRS less reliable. Eight objects have IRAC-IRS mismatches smaller than a factor 0.5; one of these objects and seven different objects have such small mismatches between MIPS and IRS (see Table 5). Slightly over one-third of these objects have noisy IRS spectra. Of the 21 objects that have either large ( $>$ factor of 2$)$ or small $(<$ factor of 0.5) mismatches, 9 are Class 0 protostars, 10 are Class I protostars, and 2 are flat-spectrum sources. In cases where the IRS flux is too high relative to the MIPS $24 \mu \mathrm{m}$ photometry, the mismatch could be due to more extended emission or flux from a nearby companion being included in the IRS measurement (SL and LL slit widths of 3". 6 and 10". 5, respectively, versus the typical FWHM of the MIPS $24 \mu \mathrm{m}$ PSF of $\sim 6^{\prime \prime}$ ).

For a few sources, the discrepancies between IRS fluxes and IRAC or MIPS can be attributed to the scaling factors applied to different parts of the IRS spectrum. As mentioned in Section 3, we typically scaled the SL spectrum to match the flux of the LL spectrum at $14 \mu \mathrm{m}$, given that the latter has a larger slit width. However, in the case of HOPS 38, where the IRAC 5.8 and $8.0 \mu \mathrm{m}$ and the MIPS $24 \mu \mathrm{m}$ fluxes are about a factor of 2.5-3.5 higher than the IRS fluxes, the LL spectrum was scaled by 0.4 to match the SL flux at $14 \mu \mathrm{m}$. Given the IRAC and MIPS measurements, it would have been more appropriate to scale the SL spectrum up. For HOPS 124, the SL spectrum was scaled by 2.5 ; if instead the LL spectrum had been scaled down, the discrepancies between IRS and IRAC and MIPS would be less than $50 \%$.

Overall, in cases where the IRS spectrum has sufficient signal-to-noise ratio and its fluxes seem lower than the photometric measurements or the discrepancies in IRAC-IRS and MIPS-IRS fluxes are quite different, intrinsic source variability could be a likely explanation. Among the sample shown in Table 5, this would apply to HOPS 24, 71, 131, 132, $141,143,154,187,206,223,228,299,363$, and 388. HOPS 223 is indeed variable (see Fischer et al. 2012), but the other objects still require confirmation. Thus, about $5 \%$ of the 290 protostars in our HOPS sample that have IRS, IRAC, and MIPS data may reveal variability to some degree. These objects are prime candidates for follow-up observations regarding their variability. 

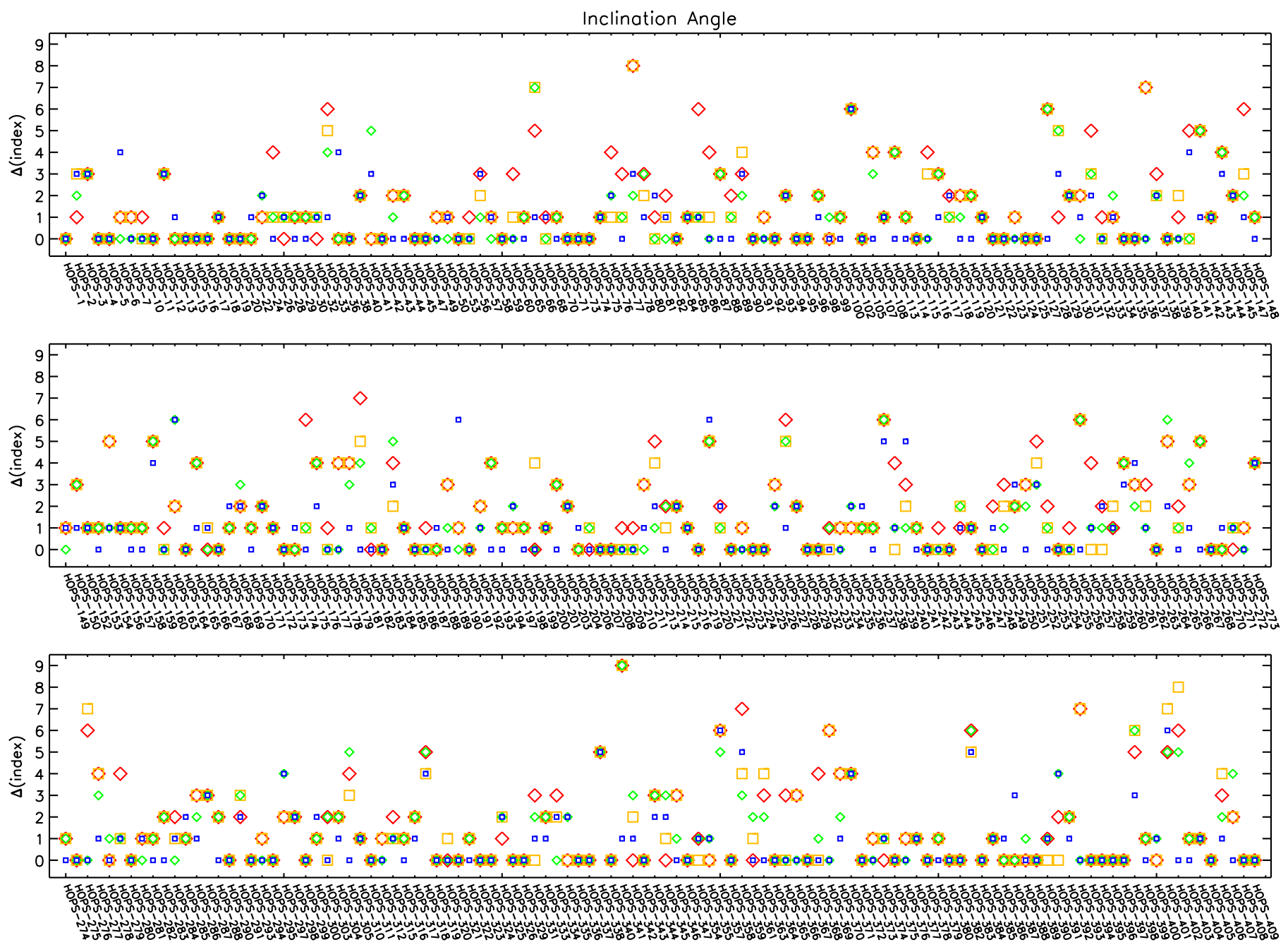

Figure 55. For all modeled HOPS targets (see name on $x$-axis), difference between the index of the best-fit inclination angle and the index of the mode of the inclination angle for models that lie within a difference of 0.5 (small blue squares), 1.0 (small green diamonds), 1.5 (yellow larger squares), and 2.0 (red larger diamonds) of the best-fit $R$.

(The complete figure set (6 images) is available online.)

\section{APPENDIX B}

\section{MODEL PARAMETER RANGES AND DEGENERACIES}

In Section 6.4 we discussed how modes can be used to assess how well model parameters are constrained. Here we analyze the spread of mode values for individual HOPS targets. In Figure Set 55 (see Figure 55 for an example) we show the difference between the modes and the best-fit values of the major model parameters $\left(i, L_{\mathrm{tot}}, \rho_{1000}, \theta, R_{\mathrm{disk}}, A_{V}\right)$ for all modeled HOPS targets. As in Section 6.4, we use models in certain $\Delta R$ bins, starting at a range of 0.5 from the best-fit $R$ up to a range of 2.0 from the best-fit $R$. For parameters with discrete values, such as the inclination and cavity opening angles, we plot the difference between the indices of modes and best-fit values. For example, if the best-fit inclination angle has a value of $41^{\circ}$ and the mode a value of $57^{\circ}$, the difference in indices would be 2 (since the discrete values in our model grid are $18^{\circ}, 32^{\circ}, 41^{\circ}, 50^{\circ}, 57^{\circ}$, etc.). Similarly, if the best-fit cavity opening angle is $5^{\circ}$ but the mode is $45^{\circ}$, the difference in indices would be 4 . For the total luminosity and foreground extinction, we plotted instead the difference between the parameter values of the best fit and the modes.

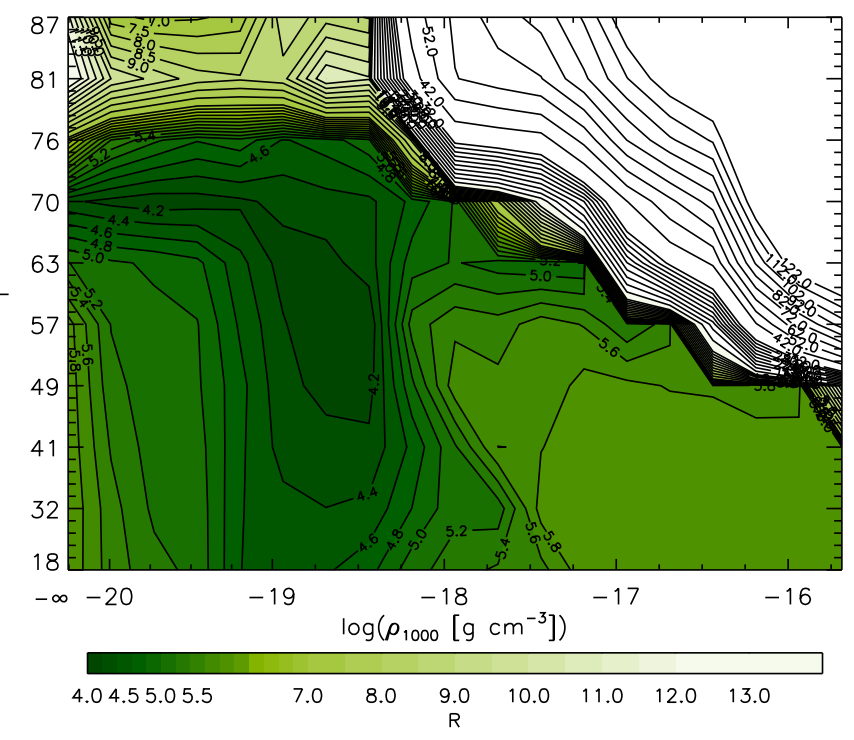

Figure 56. $R$ contour plot for the models that fit HOPS 24. For each combination of inclination angle and reference density, the lowest $R$ values of models with these two parameter values are shown.

(The complete figure set (10 images) is available online.) 

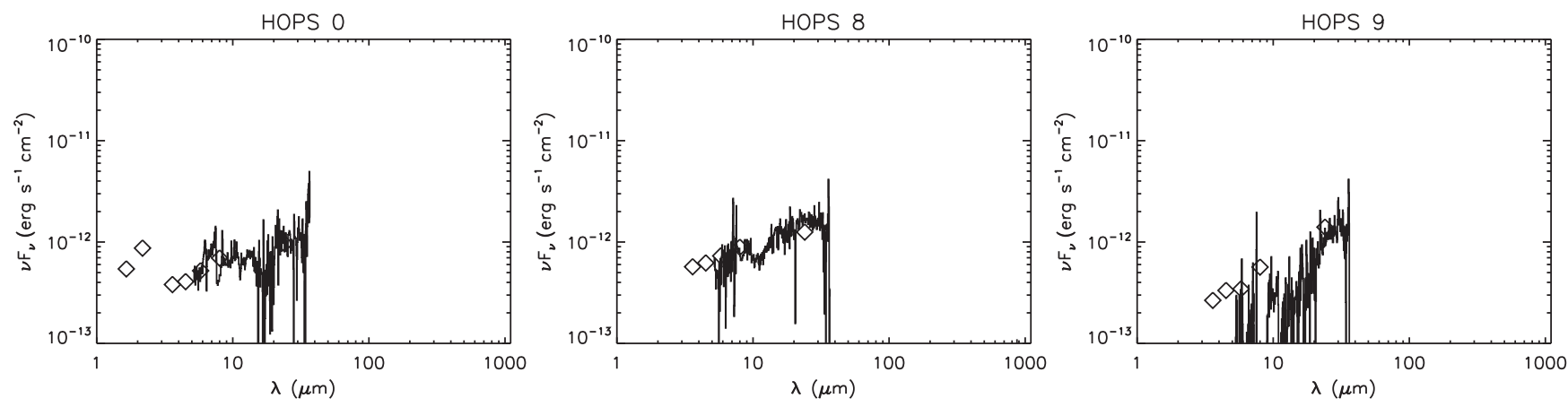

Figure 57. SEDs of the HOPS targets not modeled in this work that are likely YSOs (open symbols: photometry; line: IRS spectrum). Only the first three SEDs are shown here.

(The complete figure set (3 images) is available online.)
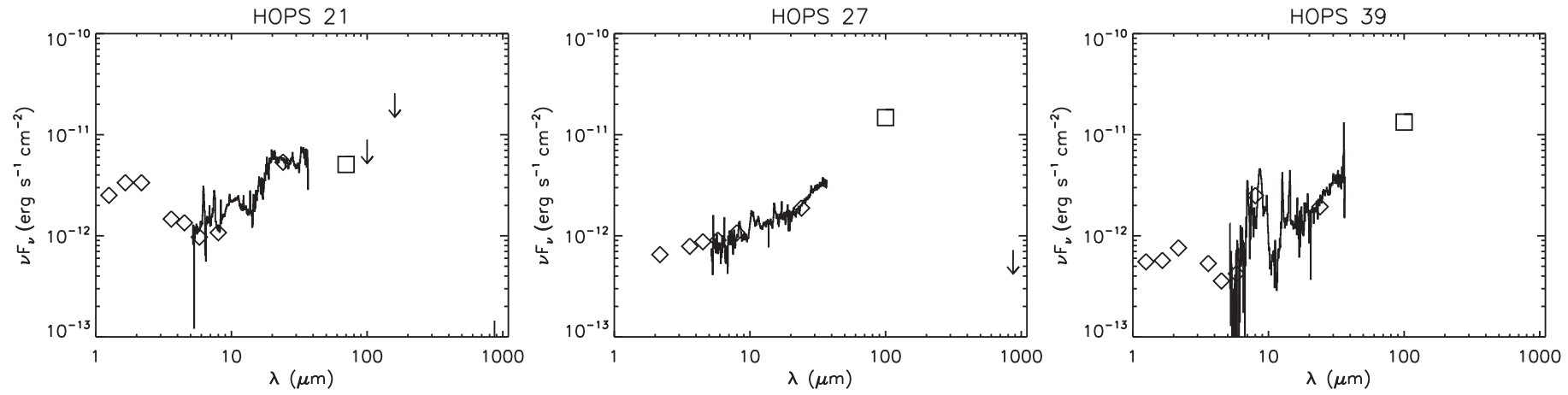

Figure 58. SEDs of the HOPS targets not modeled in this work that are likely extragalactic contaminants (open symbols: photometry; line: IRS spectrum). Only the first three SEDs are shown here.

(The complete figure set (2 images) is available online.)

Objects that are not particularly well fit by their best-fit model from the grid often have modes that are quite different from the best-fit value once $\Delta R$ reaches 2 . For example, for HOPS 181, whose best-fit model has $R=5.16$, the mode of the inclination angle for models within $\Delta R=0.5$ (i.e., models with $R<5.66)$ is the same as the best-fit value, but then the difference increases as $\Delta R$ becomes larger. For $\Delta R=2.0$, the mode is seven discrete values away from the best fit $\left(i=18^{\circ}\right.$ for the mode, $76^{\circ}$ for the best fit). Several other model parameters are also not well-constrained. There are also objects that have a relatively good fit, but a larger spread in certain parameters. An example is HOPS 70, whose best-fit model has an $R$ value of 2.33; its total luminosity and inclination angle are very well-constrained, while its reference envelope density is quite uncertain.

Certain protostars are sufficiently well-constrained by the available data and well fit by our grid of models that their parameters do not change much from $\Delta R=0.5$ to $\Delta R=2.0$. For example, the modes of the inclination angle of HOPS 1 are the same as the best-fit value even for all models within $\Delta R=2$, and the other model parameters show a small spread. There are 37 protostars with small differences between their best-fit values and modes for models within $\Delta R=2(<$ factor of two for $\rho_{1000}$ and $L_{\mathrm{to}},<50$ au for the disk radius, $<10^{\circ}$ for
Table 6

New Protostars from Stutz et al. (2013) and Tobin et al. (2015)

\begin{tabular}{|c|c|c|c|}
\hline $\begin{array}{l}\text { HOPS Identifier } \\
\text { (1) }\end{array}$ & $\begin{array}{c}\text { Original ID } \\
\text { (2) }\end{array}$ & $\begin{array}{l}\text { R.A. } \\
\left({ }^{\circ}\right) \\
(3)\end{array}$ & $\begin{array}{l}\text { Decl. } \\
\left({ }^{\circ}\right) \\
(4)\end{array}$ \\
\hline HOPS 394 & 019003 & 83.8497 & -5.1315 \\
\hline HOPS 395 & 026011 & 84.8208 & -7.4074 \\
\hline HOPS 396 & 029003 & 84.8048 & -7.2199 \\
\hline HOPS 397 & 061012 & 85.7036 & -8.2696 \\
\hline HOPS 398 & 082005 & 85.3725 & -2.3547 \\
\hline HOPS 399 & 082012 & 85.3539 & -2.3024 \\
\hline HOPS 400 & 090003 & 85.6885 & -1.2706 \\
\hline HOPS 401 & 091015 & 86.5319 & -0.2058 \\
\hline HOPS 402 & 091016 & 86.5415 & -0.2047 \\
\hline HOPS 403 & 093005 & 86.6156 & -0.0149 \\
\hline HOPS 404 & 097002 & 87.0323 & 0.5641 \\
\hline HOPS 405 & 119019 & 85.2436 & -8.0934 \\
\hline HOPS 406 & 300001 & 86.9307 & 0.6396 \\
\hline HOPS 407 & 302002 & 86.6177 & 0.3242 \\
\hline HOPS 408 & 313006 & 84.8781 & -7.3998 \\
\hline HOPS 409 & 135003 & 83.8392 & -5.2215 \\
\hline
\end{tabular}

Note. Column (1) lists the HOPS number of the object, column (2) the identifier of the source from Stutz et al. (2013) and Tobin et al. (2015), and columns (3) and (4) its J2000 coordinates in degrees. 
Table 7

YSOs in the HOPS Sample with No PACS Data

\begin{tabular}{|c|c|c|c|c|c|c|c|}
\hline Object & $\begin{array}{l}\text { R.A. } \\
\left({ }^{\circ}\right)\end{array}$ & $\begin{array}{c}\text { Decl. } \\
\left({ }^{\circ}\right)\end{array}$ & Class & $\begin{array}{l}L_{\mathrm{bol}} \\
\left(L_{\odot}\right)\end{array}$ & $\begin{array}{l}T_{\text {bol }} \\
(\mathrm{K})\end{array}$ & $n_{4.5-24}$ & PACS Flag \\
\hline (1) & (2) & (3) & (4) & (5) & (6) & (7) & (8) \\
\hline HOPS 0 & 88.6171 & 1.6264 & I & 0.011 & 652.2 & 0.514 & $\overline{-1}$ \\
\hline HOPS 8 & 83.8880 & -5.9851 & I & 0.013 & 329.8 & 0.419 & 0 \\
\hline HOPS 9 & 83.9550 & -5.9843 & I & 0.006 & 281.5 & 0.858 & -1 \\
\hline HOPS 14 & 84.0799 & -5.9251 & flat & 0.042 & 464.0 & 0.246 & 0 \\
\hline HOPS 23 & 84.0745 & -5.7818 & I & 0.012 & 346.8 & 0.539 & 0 \\
\hline HOPS 25 & 83.8443 & -5.7415 & flat & 0.045 & 646.6 & 0.165 & 0 \\
\hline HOPS 31 & 83.8219 & -5.6741 & flat & 0.024 & 634.7 & 0.304 & 0 \\
\hline HOPS 34 & 83.7954 & -5.6585 & I & 0.013 & 235.5 & 0.762 & 0 \\
\hline HOPS 35 & 83.8331 & -5.6503 & I & 0.044 & 305.2 & 0.884 & 0 \\
\hline HOPS 37 & 83.6986 & -5.6237 & flat & 0.016 & 913.4 & 0.230 & 0 \\
\hline HOPS 51 & 83.8160 & -5.5015 & II & 0.518 & 130.2 & $\ldots$ & 0 \\
\hline HOPS 52 & 83.8180 & -5.4924 & flat & 0.641 & 610.8 & -0.163 & 0 \\
\hline HOPS 54 & 83.3437 & -5.3841 & II & 0.097 & 1879.3 & -0.37 & -1 \\
\hline HOPS 62 & 83.8524 & -5.1916 & flat & 0.660 & 1154.1 & 0.043 & 0 \\
\hline HOPS 63 & 83.8538 & -5.1671 & flat & 0.516 & 544.5 & 0.004 & 0 \\
\hline HOPS 64 & 83.8625 & -5.1650 & I & 15.347 & 29.7 & 0.503 & 0 \\
\hline HOPS 69 & 83.8551 & -5.1400 & flat & 2.778 & 31.3 & -0.189 & 0 \\
\hline HOPS 79 & 83.8662 & -5.0934 & flat & 0.086 & 666.2 & -0.137 & 0 \\
\hline HOPS 103 & 83.5508 & -4.8353 & flat & 0.142 & 1484.3 & -0.032 & 0 \\
\hline HOPS 104 & 83.7782 & -4.8338 & I & 0.044 & 337.3 & 0.837 & 0 \\
\hline HOPS 110 & 84.0093 & -5.0472 & I & 0.014 & 244.0 & $\ldots$ & -1 \\
\hline HOPS 126 & 85.0408 & -7.1650 & flat & 0.132 & 1865.3 & -0.136 & -1 \\
\hline HOPS 151 & 84.6787 & -6.9447 & II & 0.061 & 799.4 & -0.505 & 0 \\
\hline HOPS 155 & 84.3160 & -7.2972 & flat & 0.013 & 393.8 & 0.133 & -1 \\
\hline HOPS 162 & 84.1291 & -6.8780 & II & 0.015 & 909.9 & 0.352 & -1 \\
\hline HOPS 180 & 84.2475 & -6.1710 & II & 0.011 & 1493.5 & 0.578 & -1 \\
\hline HOPS 195 & 84.0002 & -6.1206 & flat & 0.032 & 659.7 & 0.399 & 0 \\
\hline HOPS 217 & 85.7965 & -8.4056 & I & 0.008 & 323.8 & 0.773 & -1 \\
\hline HOPS 230 & 85.6283 & -8.1515 & flat & 0.267 & 1260.2 & -0.104 & -1 \\
\hline HOPS 231 & 85.1189 & -8.5486 & flat & 0.024 & 386.0 & -0.239 & -1 \\
\hline HOPS 269 & 85.3625 & -7.7094 & flat & 0.025 & 230.2 & 0.023 & -1 \\
\hline HOPS 289 & 84.9865 & -7.5017 & I & 0.095 & 331.1 & 0.868 & 0 \\
\hline HOPS 296 & 85.3215 & -2.3021 & I & 0.022 & 326.0 & 0.931 & 0 \\
\hline HOPS 302 & 85.0934 & -2.2610 & flat & 0.383 & 1367.2 & -0.032 & -1 \\
\hline HOPS 307 & 85.3077 & -1.7844 & 0 & 0.748 & 57.1 & 1.506 & -1 \\
\hline HOPS 314 & 86.6505 & -0.3414 & I & 0.015 & 276.2 & 1.112 & -1 \\
\hline HOPS 327 & 86.6139 & 0.1477 & flat & 0.020 & 991.0 & 0.145 & -1 \\
\hline HOPS 328 & 86.5561 & 0.1759 & I & 0.012 & 326.3 & 0.868 & -1 \\
\hline HOPS 330 & 86.7140 & 0.3298 & flat & 0.121 & 385.2 & 0.285 & 0 \\
\hline HOPS 332 & 86.8821 & 0.3391 & flat & 0.249 & 145.5 & 0.045 & 0 \\
\hline HOPS 360 & 86.8629 & 0.3425 & I & 1.017 & 43.2 & $\ldots$ & 0 \\
\hline
\end{tabular}

Note. Column (1) lists the HOPS number of the object, columns (2) and (3) its J2000 coordinates in degrees, column (4) the type based on SED classification, column (5) the bolometric luminosity, column (6) the bolometric temperature, column (7) the 4.5-24 $\mu \mathrm{m}$ SED slope, and column (8) a flag identifying whether the object was not observed by PACS (flag value of -1 ) or not detected by PACS at $70 \mu \mathrm{m}$ (flag value of 0 ).

the cavity opening angle, $<30 \%$ difference in inclination angle). These protostars are well characterized by our model fits. The mean and median $R$ values for their best-fit models are 3.48 and 3.17 , respectively. This validates our estimate of $R \sim 4$ as the boundary between a reliable and a less reliable fit.

Part of the parameter uncertainties can be attributed to degeneracies between model parameters. To illustrate some of these degeneracies, in Figure Set 56 (see Figure 56 for an example) we show contour plots of $R$ values for sets of two model parameters each (we plot the lowest $R$ value of models with these two parameter values) resulting from the model fits of HOPS 24, HOPS 107, and HOPS 149. The plots for HOPS 24 show that the inclination angle is somewhat degenerate with the envelope density, with higher inclination angles being accommodated by lower $\rho_{1000}$ values (Figure 56). A similar situation applies to the disk radius, with larger disk radii requiring higher envelope densities. The inclination angle and the cavity opening angle are degenerate, too; for higher inclination angles the cavity is larger. The $R$ contour plots for HOPS 107 suggest that a certain range of inclination angles and reference densities can fit the SED, while the disk radius and cavity opening angles are not well-constrained. However, the plots clearly show that high-density, high-inclination models fit very poorly. Finally, we can deduce from the $R$ contour plots for HOPS 149 that also here certain parameter values can be excluded; lower inclination angles and reference densities in the $10^{-18}-10^{-19} \mathrm{~g} \mathrm{~cm}^{-3}$ range yield the best fits, with larger densities accompanied by larger disk radii and larger cavity opening angles. 


\section{APPENDIX C NOTES ON HOPS TARGETS}

\section{C.1. HOPS Targets Discovered with Herschel}

Table 6 lists the newest HOPS targets discovered in Herschel PACS data (HOPS 394 to 409; Stutz et al. 2013; Tobin et al. 2015 ) with their coordinates and original identifiers from Stutz et al. (2013).

\section{C.2. HOPS Objects Not Included in the Modeling Sample}

\section{C.2.1. Young Stellar Objects}

Among our HOPS sample, there are 41 targets that are likely YSOs, but they lack PACS measurements at 70 and $160 \mu \mathrm{m}$ and were therefore not included in the modeling sample. There are four additional targets with HOPS numbers that were not modeled; they are HOPS 109, 111, 212, and 362, and they are duplicates of HOPS 40, 60, 211, and 169, respectively. Table 7 lists the 41 likely YSOs in the HOPS catalog that were not part of the modeling sample; their SEDs are shown in Figure Set 57. Among them, 17 were not observed by PACS at $70 \mu \mathrm{m}$, while 24 were observed, but not detected at $70 \mu \mathrm{m}$. The majority of these targets are Class I protostars or flat-spectrum sources; only one is a Class 0 protostar, and five are Class II pre-mainsequence stars.

Most of these YSOs have very faint fluxes in the near- to mid-IR. They could be deeply embedded protostars, like HOPS 307 , or just very low mass protostars with weak envelope emission. Objects with little excess emission out to about $8 \mu \mathrm{m}$, a $10 \mu \mathrm{m}$ silicate emission feature, and a more or less steeply rising SED beyond $12 \mu \mathrm{m}$ are likely transitional disks (see Kim et al. 2013); good examples are HOPS 51 and 54. It is possible that some of the YSOs in this sample are actually extragalactic contaminants, in particular objects with flat SEDs (see more about this subset of our HOPS sample in the next subsection).

\section{C.2.2. Contaminants}

Our HOPS sample contains 29 targets that turned out to be likely extragalactic contaminants. These objects are listed in Table 8, and their SEDs are shown in Figure Set 58. Most galaxies were identified based on the presence of PAH features or emission lines in their IRS spectra (in particular, the $5-15 \mu \mathrm{m}$ region), the absence of a silicate absorption feature at $10 \mu \mathrm{m}$, and an overall flat or slightly rising midinfrared continuum. Clear examples are HOPS 21, 27, 46, 48, $55,61,72,106,161$, and 301 .

Two objects, HOPS 202 and HOPS 205, have a tentative $10 \mu \mathrm{m}$ silicate emission feature and a steep rise of their SED beyond $12 \mu \mathrm{m}$, but also PAH emission features in their IRS spectrum; they could be transitional disks and not galaxies.

In some cases, e.g., HOPS 308, targets classified as extragalactic contaminants are very faint in the near- to midinfrared; instead of galaxies, they could be very low mass or deeply embedded protostars.

The mid-IR SED of HOPS 339 is mostly flat but displays a sharp $10 \mu \mathrm{m}$ absorption feature; based on the SED alone, it would not necessarily be classified as a galaxy, but highresolution near-IR HST images resolve its extended emission and reveal a spiral galaxy (J. Booker et al. 2016, in preparation).
Table 8

Likely Extragalactic Contaminants in the HOPS Sample

\begin{tabular}{|c|c|c|c|c|c|}
\hline Object & $\begin{array}{c}\text { R.A. } \\
\left({ }^{\circ}\right) \\
(2)\end{array}$ & $\begin{array}{c}\text { decl. } \\
\left({ }^{\circ}\right) \\
(3)\end{array}$ & $\begin{array}{c}L_{\text {bol }} \\
\left(L_{\odot}\right) \\
(4)\end{array}$ & $\begin{array}{l}T_{\text {bol }} \\
(\mathrm{K}) \\
(5)\end{array}$ & $\begin{array}{c}n_{4.5-24} \\
(6)\end{array}$ \\
\hline HOPS 21 & 84.0421 & -5.8356 & 0.074 & 584.5 & 0.824 \\
\hline HOPS 27 & 84.0905 & -5.6995 & 0.102 & 118.2 & 0.458 \\
\hline HOPS 39 & 84.0934 & -5.6069 & 0.100 & 159.4 & 1.010 \\
\hline HOPS 46 & 83.6758 & -5.5509 & 0.141 & 1081.9 & -0.083 \\
\hline HOPS 48 & 83.7773 & -5.5477 & 0.608 & 611.0 & 0.252 \\
\hline HOPS 55 & 83.4754 & -5.3638 & 0.316 & 101.5 & 1.732 \\
\hline HOPS 61 & 83.3579 & -5.2007 & 0.045 & 721.4 & -0.031 \\
\hline HOPS 67 & 83.8445 & -5.1428 & 0.044 & 278.7 & 0.900 \\
\hline HOPS 72 & 83.8571 & -5.1296 & 0.545 & 693.0 & 0.068 \\
\hline HOPS 83 & 83.9822 & -5.0771 & 0.131 & 293.9 & -0.119 \\
\hline HOPS 97 & 83.8704 & -4.9608 & 0.190 & 403.8 & 0.125 \\
\hline HOPS 101 & 83.7843 & -4.9027 & 3.355 & 481.2 & -0.207 \\
\hline HOPS 106 & 84.0518 & -4.7544 & 0.016 & 359.7 & 0.943 \\
\hline HOPS 112 & 85.1833 & -7.3786 & 0.014 & 390.2 & 0.093 \\
\hline HOPS 146 & 84.6840 & -7.0112 & 0.053 & 519.7 & -0.178 \\
\hline HOPS 161 & 84.1448 & -7.1871 & 0.062 & 179.1 & 0.458 \\
\hline HOPS 196 & 83.8371 & -6.3062 & 0.065 & 165.5 & -0.042 \\
\hline HOPS 202 & 83.4330 & -6.2295 & 0.018 & 736.3 & 0.534 \\
\hline HOPS 205 & 85.7620 & -8.7971 & 0.067 & 427.8 & 0.549 \\
\hline HOPS 218 & 85.7912 & -8.2232 & 0.007 & 332.5 & 0.653 \\
\hline HOPS 292 & 84.4787 & -7.6890 & 0.068 & 280.5 & 0.421 \\
\hline HOPS 301 & 85.4366 & -2.2654 & 2.955 & 518.8 & 0.271 \\
\hline HOPS 306 & 85.7630 & -1.8013 & 0.038 & 310.8 & 0.060 \\
\hline HOPS 308 & 85.8082 & -1.7195 & 0.042 & 156.0 & 0.791 \\
\hline HOPS 309 & 85.6973 & -1.4131 & 0.041 & 111.9 & -0.168 \\
\hline HOPS 313 & 85.2532 & -1.1529 & 0.030 & 154.1 & 1.039 \\
\hline HOPS 339 & 86.4733 & 0.4243 & 0.128 & 398.3 & 0.246 \\
\hline HOPS 348 & 86.7511 & 0.3438 & 0.286 & 84.0 & $\ldots$ \\
\hline HOPS 351 & 83.8809 & -5.0797 & 0.016 & 217.1 & $\ldots$ \\
\hline
\end{tabular}

Note. Column (1) lists the HOPS number of the object, columns (2) and (3) its J2000 coordinates in degrees, column (4) the bolometric luminosity, column (5) the bolometric temperature, and column (6) the 4.5-24 $\mu \mathrm{m}$ SED slope.

Table 9

Targets in the HOPS Sample with Uncertain Nature

\begin{tabular}{lcc}
\hline \hline $\begin{array}{l}\text { Object } \\
(1)\end{array}$ & $\begin{array}{c}\text { R.A. } \\
(2)\end{array}$ & $\begin{array}{c}\text { Decl. } \\
(3)\end{array}$ \\
\hline HOPS 349 & 83.8592 & -5.1426 \\
HOPS 350 & 83.8758 & -5.1386 \\
HOPS 352 & 83.8617 & -5.0675 \\
HOPS 353 & 88.5556 & 1.7175 \\
HOPS 356 & 85.5341 & -1.4438 \\
HOPS 381 & 83.7816 & -5.6986 \\
\hline
\end{tabular}

Note. Column (1) lists the HOPS number of the object, columns (2) and (3) its J2000 coordinates in degrees.

Finally, HOPS $349,350,352,353,356$, and 381 have poorly sampled SEDs (just one or two flux measurements), so their nature is quite uncertain. We list their coordinates in Table 9.

\section{REFERENCES}

Adams, F. C., Lada, Ch. J., \& Shu, F. H. 1987, ApJ, 312, 788

Adams, F. C., \& Shu, F. H. 1986, ApJ, 308, 836

Adams, J. D., Herter, T. L., Osorio, M., et al. 2012, ApJL, 749, L24 
Ali, B., Tobin, J. J., Fischer, W. J., et al. 2010, A\&A, 518, L119 André, P., Men'shchikov, A., Bontemps, S., et al. 2010, A\&A, 518, L102 André, P., \& Montmerle, T. 1994, ApJ, 420, 837

André, P., Ward-Thompson, D., \& Barsony, M. 1993, ApJ, 406, 122 Arce, H. G., \& Sargent, A. I. 2006, ApJ, 646, 1070

Billot, N., Morales-Calderón, M., Stauffer, J. R., et al. 2012, ApJL, 753, L35 Boogert, A. C. A., Pontoppidan, K. M., Knez, C., et al. 2008, ApJ, 678, 985 Calvet, N., Hartmann, L., Kenyon, S. J., \& Whitney, B. A. 1994, ApJ, 434, 330 Caratti o Garatti, A., Garcia Lopez, R., Scholz, A., et al. 2011, A\&A, 526, L1 Cassen, P., \& Moosman, A. 1981, Icar, 48, 353

Chen, H., Myers, P. C., Ladd, E. F., \& Wood, D. O. S. 1995, ApJ, 445, 377

Cody, A. M., Stauffer, J., Baglin, A., et al. 2014, AJ, 147, 82

Cohen, M., Wheaton, W. A., \& Megeath, S. T. 2003, AJ, 126, 1090

Crapsi, A., van Dishoeck, E. F., Hogerheijde, M. R., et al. 2008, A\&A, 486, 245

di Francesco, J., Evans, N. J., II, Caselli, P., et al. 2007, in Protostars \& Planets V, ed. B. Reipurth, D. Jewitt, \& K. Keil (Tucson: Univ. Arizona Press), 17 Draine, B. T. 2003, ARA\&A, 41, 241

Dunham, M. M., Allen, L. E., Evans, N. J., II, et al. 2015, ApJS, 220, 11

Dunham, M. M., Arce, H. G., Allen, L. E., et al. 2013, AJ, 145, 94

Dunham, M. M., Crapsi, A., Evans, N. J., II, et al. 2008, ApJS, 179, 249

Dunham, M. M., Evans, N. J., II, Terebey, S., et al. 2010, ApJ, 710, 470

Dunham, M. M., Stutz, A. M., Allen, L. E., et al. 2014, in Protostars \& Planets VI, ed. H. Beuther et al. (Tucson: Univ. Arizona Press), 195

Dunham, M. M., \& Vorobyov, E. I. 2012, ApJ, 747, 52

Enoch, M., Evans, N. J., II, Sargent, A. I., \& Glenn, J. 2009, ApJ, 692, 973

Engelbracht, C. W., Blaylock, M., Su, K. Y. L., et al. 2007, PASP, 119, 994

Espaillat, C., Muzerolle, J., Najita, J., et al. 2014, in Protostars \& Planets VI, ed. H. Beuther et al. (Tucson: Univ. Arizona Press), 497

Evans, N. J., II, Dunham, M. M., Jørgensen, J. K., et al. 2009, ApJS, 181, 321

Evans, N. J., II, Rawlings, J. M. C., Shirley, Y. L., \& Mundy, L. G. 2001, ApJ, 557,193

Fazio, G. G., Hora, J. L., Allen, L. E., et al. 2004, ApJS, 154, 10

Fischer, W. J., Megeath, S. T., Ali, B., et al. 2010, A\&A, 518, L122

Fischer, W. J., Megeath, S. T., Stutz, A. M., et al. 2013, AN, 334, 53

Fischer, W. J., Megeath, S. T., Tobin, J. J., et al. 2012, ApJ, 756, 99

Fischer, W. J., Megeath, S. T., Tobin, J. J., et al. 2014, ApJ, 781, 123

Furlan, E., McClure, M., Calvet, N., et al. 2008, ApJS, 176, 184

Furlan, E., Megeath, S. T., Osorio, M., et al. 2014, ApJ, 786, 26

Greene, T. P., Wilking, B. A., André, P., et al. 1994, ApJ, 434, 614

Günther, H. M., Cody, A. M., Covey, K. R., et al. 2014, AJ, 148, 122

Hartmann, L., Calvet, N., \& Boss, A. , 1996, ApJ, 464, 387

Heiderman, A., \& Evans, N. J., II 2015, ApJ, 806, 231

Houck, J. R., Roellig, T. L., van Cleve, J., et al. 2004, ApJS, 154, 18

Kenyon, S. J., Calvet, N., \& Hartmann, L. 1993, ApJ, 414, 676

Kenyon, S. J., Hartmann, L. W., Strom, K. M., \& Strom, S. E. 1990, AJ, 99,869

Kim, K. H., Watson, D. M., Manoj, P., et al. 2013, ApJ, 769, 149

Kim, K. H., Watson, D. M., Manoj, P., et al. 2016, ApJS, submitted

Kim, M. K., Hirota, T., Honma, M., et al. 2008, PASJ, 60, 991

Kounkel, M., Megeath, S. T., Poteet, C. A., et al. 2016, ApJ, in press (arXiv:1602.07635)

Kryukova, E., Megeath, S. T., Gutermuth, R. A., et al. 2012, AJ, 144, 31

Kryukova, E., Megeath, S. T., Hora, J. L., et al. 2014, AJ, 148, 11

Lada, Ch. J. 1987, in Proc. IAU Symp. 115, Star Forming Regions, ed. M. Peimbert, \& J. Jugaku (Dordrecht: Reidel), 1

Launhardt, R., Stutz, A. M., Schmiedeke, A., et al. 2013, A\&A, 551, A98
Lim, J., \& Takakuwa, S. 2006, ApJ, 653, 425

Manoj, P., Watson, D. M., Neufeld, D. A., et al. 2013, ApJ, 763, 83

Mathis, J. S. 1990, ARA\&A, 28, 37

Mathis, J. S., Mezger, P. G., \& Panagia, N. 1983, A\&A, 128, 212

Maury, A. J., André, P., Men'shchikov, A., et al. 2011, A\&A, 535, A77

McClure, M. 2009, ApJL, 693, L81

Megeath, S. T., Gutermuth, R., Muzerolle, J., et al. 2012, AJ, 144, 192

Menten, K. M., Reid, M. J., Forbrich, J., \& Brunthaler, A. 2007, A\&A, 474,515

Morales-Calderón, M., Stauffer, J. R., Hillenbrand, L. A., et al. 2011, ApJ, 733,50

Myers, P. C., \& Ladd, E. F. 1993, ApJL, 413, L47

Offner, S. S. R., \& McKee, C. F. 2011, ApJ, 736, 53

Ormel, C. W., Min, M., Tielens, A. G. G. M., Dominik, C., \& Paszun, D. 2011, A\&A, 532, A43

Ossenkopf, V., \& Henning, T. 1994, A\&A, 291, 943

Pilbratt, G. L., Riedinger, J. R., Passvogel, T., et al. 2010, A\&A, 518, L1

Pillitteri, I., Wolk, S. J., Megeath, S. T., et al. 2013, ApJ, 768, 99

Poglitsch, A., Waelkens, C., Geis, N., et al. 2010, A\&A, 518, L2

Pontoppidan, K. M., Boogert, A. C. A., Fraser, H. J., et al. 2008, ApJ, 678, 1005

Poppenhaeger, K., Cody, A. M., \& Covey, K. R. 2015, AJ, 150, 118

Poteet, C. A., Megeath, S. T., Watson, D. M., et al. 2011, ApJL, 733, L32

Reach, W. T., Megeath, S. T., Cohen, M., et al. 2005, PASP, 117, 978

Rebull, L. M., Cody, A. M., Covey, K. R., et al. 2014, AJ, 148, 92

Rebull, L. M., Stauffer, J. R., Cody, A. M., et al. 2015, AJ, 150, 175

Rieke, G. H., Young, E. T., Engelbracht, C. W., et al. 2004, ApJS, 154, 25

Robitaille, T. P., Whitney, B. A., Indebetouw, R., et al. 2006, ApJS, 167, 256

Rodríguez, L. F., D’Alessio, P., Wilner, D. J., et al. 1998, Natur, 395, 355

Sadavoy, S. I., Di Francesco, J., André, P., et al. 2014, ApJL, 787, L18

Safron, E. J., Fischer, W. J., Megeath, S. T., et al. 2015, ApJL, 800, L5

Shirley, Y. L., Evans, N. J., II, \& Rawlings, J. M. C. 2002, ApJ, 575, 337

Siringo, G., Kreysa, E., De Breuck, C., et al. 2010, Msngr, 139, 20

Siringo, G., Kreysa, E., Kovács, A., et al. 2009, A\&A, 497, 945

Skrutskie, M. F., Cutri, R. M., Stiening, R., et al. 2006, AJ, 131, 1163

Stanke, T., Stutz, A. M., Tobin, J. J., et al. 2010, A\&A, 518, L94

Stutz, A. M., \& Kainulainen 2015, A\&A, 577, L6

Stutz, A. M., Launhardt, R., Linz, H., et al. 2010, A\&A, 518, L87

Stutz, A. M., Tobin, J. J., Stanke, T., et al. 2013, ApJ, 767, 36

Terebey, S., Shu, F. H., \& Cassen, P. 1984, ApJ, 286, 529

Tobin, J. J., Hartmann, L., Bergin, E., et al. 2012, ApJ, 748, 16

Tobin, J. J., Hartmann, L., Calvet, N., \& D'Alessio, P. 2008, ApJ, 679,1364

Tobin, J. J., Stutz, A. M., Megeath, S. T., et al. 2015, ApJ, 798, 128

Ulrich, R. K. 1976, ApJ, 210, 377

Werner, M. W., Roellig, T. L., Low, F. J., et al. 2004, ApJS, 154, 1

Whitney, B. A., Robitaille, T. P., Bjorkman, J. E., et al. 2013, ApJS, 207,30

Whitney, B. A., Wood, K., Bjorkman, J. E., \& Cohen, M. 2003a, ApJ, 598, 1079

Whitney, B. A., Wood, K., Bjorkman, J. E., \& Wolff, M. J. 2003b, ApJ, 591, 1049

Winston, E., Megeath, S. T., Wolk, S. J., et al. 2010, AJ, 140, 266

Wolk, S. J., Günther, H. M., Poppenhaeger, K., et al. 2015, AJ, 150, 145

Young, C. H., \& Evans, N. J., II 2005, ApJ, 627, 293

Young, C. H., Shirley, Y. L., Evans, N. J., II, \& Rawlings, J. M. C. 2003, ApJS, 145, 111 